\title{
Circuit Theory of Linear Noisy Netwonks
}





\section{WOODS MOLE \\ OCEANOGRAPHIC INSTITUTION}

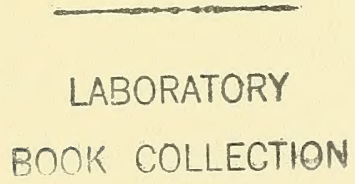

Purchaso Ord or No... 0219 

Circuit Theory

of Linear Noisy Networks 


\section{TECHNOLOGY PRESS RESEARCH MONOGRAPHS}

Nontinear Problems in Random Theory By Norbert Wiener

Circuit Theory of Linear Noisy Networks By Hermann A. Haus and Richard B. Adler 
by

HERMANN A. HAUS

RICHARD B. ADLER

Associate Professors of Electrical Engineering

Massachusetts Institute of Technology

Circuit Theory

of Linear Noisy Networks

Published jointly by

The Technology Press of

The Massachusetts Institute of Technology

and 
Copyright (C) 1959

by

The Massachusetts Institute of Technology

\section{All Rights Reserved}

This book or any part thereof must not be reproduced in any form without the written permission of the publisher.

Library of Congress Catalog Card Number: 59-11473

Printed in the United States of America 


\section{Foreword}

There has long been a need in science and engineering for systematic publication of research studies larger in scope than a journal article but less ambitious than a finished book. Much valuable work of this kind is now published only in a semiprivate way, perhaps as a laboratory report, and so may not find its proper place in the literature of the field. The present contribution is the second of the Technology Press Research Monographs, which we hope will make selected timely and important research studies readily accessible to libraries and to the independent worker.

J. A. Stratton 



\section{Preface}

Monographs usually present scholarly summaries of a well-developed field. In keeping with the philosophy of the new series of Research Monographs, however, this monograph was written to present a piece of relatively recent work in a comparatively undeveloped field. Such work might normally be expected to appear in a series of journal articles, and indeed originally the authors followed this method of presentation. As the subject developed, however, a rather general approach to the problem became apparent which both simplified and unified all the prior research. Space limitations in the journals made it impossible to publish in that medium a really suitable picture of the whole development, and this circumstance led the authors to take advantage of the present Technology Press Research Monographs.

The principal motivation for this work arose from the obvious desirability of finding a single quantity, a tag so to speak, to describe the noise performance of a two-terminal-pair amplifier. The possibility of the existence of such a quantity and even the general functional form which it might be expected to take were suggested by previous work of one of the authors on microwave tubes and their noise performance. This work showed that noise parameters of the electron beam set an ultimate limit to the entire noise performance of the amplifier that employed the beam. In the microwave tube case, however, the findings were based heavily upon the physical nature of the electron beam, and it was not immediately clear that a general theory of noise performance 
for any linear amplifier could be made without referring again to some detailed physical mechanism. In order to detach the study of noise performance from specific physical mechanisms, one had to have recourse to general circuit theory of active networks. Such a theory had grown up around the problems associated with transistor amplifiers, and important parts of it were available to us through the association of one of us with Professor S. J. Mason. This combination of circumstances led to the collaboration of the authors.

Two major guiding principles, or clues, could be drawn from the experience on microwave tubes. One such clue was the general form of the probable appropriate noise parameter. The other was the recognition that matrix algebra and a proper eigenvalue formulation would be required in order to achieve a general theory without becoming hopelessly involved in algebraic detail.

Essentially by trial and error, guided by some power-gain theorems in active circuit theory, we first found a few invariants of noisy networks. Afterward, while we were trying to decide around which quantities we should build a matrix-eigenvalue formulation leading to these same invariants, we were aided by the fact that Mr. D. L. Bobroff recognized a connection between the invariants which we had found and the problem of the available power of a multiterminal-pair network.

Armed with this additional idea, we consulted extensively with Professor L. N. Howard of the Massachusetts Institute of Technology, Department of Mathematics, in search of the appropriate matrix-eigenvalue problem. As a result of his suggestions, we were able to reach substantially the final form of the desired formulation.

Once the proper eigenvalue approach was found, additional results and interpretations followed rapidly. In particular, the idea that the eigenvalue formulation should be associated with a canonical form of the noisy network was suggested in a conversation with Professor Shannon.

One of the principal results of the work is that it furnishes a single number, or tag, which may be said to characterize the amplifier noise performance on the basis of the signal-to-noise-ratio criterion. The novel features of this tag are two in number: First, it clears up questions of the noise performance of low-gain amplifiers or of the effect upon noise performance of degenerative feedback; second, it provides for the first time a systematic treatment of the noise performance of negative-resistance amplifiers. The latter results were not expected in the original motivation for the study but grew from insistent demands upon the internal consistency of the theory. It is interesting that the negativeresistance case will probably turn out to be one of the most important practical results of our work. 
Another result worth mentioning here, however, is the canonical form of linear noisy networks. This form summarizes in a clear, almost visual, manner the connection between the internal noise of a network at any particular frequency and its (resistive, positive, or negative) part.

We are hopeful that this second work in the series of Technology Press Research Monographs will meet the standards and aims envisioned by Professor Gordon S. Brown, whose personal inspiration and energetic support brought the present volume into existence.

We wish to express our sincere thanks to Miss Joan Dordoni for the careful preparation of the manuscript. We also acknowledge gratefully the thorough and exacting editing work of Miss Constance D. Boyd.

The support in part by the U. S. Army (Signal Corps), the U. S. Air Force (Office of Scientific Research, Air Research and Development Command), and the U. S. Navy (Office of Naval Research) is also acknowledged with gratitude.

Massachusetts Institute of Technology

Cambridge, Massachusetts

January, 1959 



\section{Contents}

FOREWORD

PREFACE

vii

CHAPTER 1 Introduction 1

CHAPTER 2 Linear Noisy Networks in the Impedance Representation 9

2.1 Impedance-Matrix Representation of Linear Networks 9

2.2 Lossless Transformations 12

2.3 Network Classification in Terms of Power 14

CHAPTER 3 Impedance Formulation of the Characteristic-Noise Matrix 19

3.1 Matrix Formulation of Stationary-Value Problem 19

3.2 Eigenvalue Formulation of Stationary-Value Problem 21

3.3 Properties of the Eigenvalues of the CharacteristicNoise Matrix in Impedance Form 23

3.4 Lossless Reduction in the Number of Terminal Pairs 24

CHAPTER 4 Canonical Form of Linear Noisy Networks 28

4.1 Derivation of the Canonical Form 28

4.2 Interconnection of Linear Noisy Networks 31

CHAPTER 5 Linear Noisy Networks in Other Representations 33

5.1 General Matrix Representations 33

5.2 Transformation from One Matrix Representation to Another 
5.3 Power Expression and Its Transformation 37

5.4 The General Characteristic-Noise Matrix 38

CHAPTER 6 Noise Measure 42

6.1 Extended Definitions of Gain and Noise Figure 43

6.2 Matrix Formulation of Exchangeable Power and Noise Figure

6.3 Noise Measure 48

6.4 Allowed Ranges of Values of the Noise Measure 49

6.5 Arbitrary Passive Interconnection of Amplifiers 54

ChAPter 7 Network Realization of Optimum Amplifier Noise Performance 58

7.1 Classification of Two-Terminal-Pair Amplifiers 59

7.2 Optimization of Amplifier, Indefinite Case 61

7.3 The Optimum Noise-Measure Expression for the Conventional Low-Frequency Vacuum Tube 66

7.4 Optimization of Negative-Resistance Amplifiers, Definite Case 68

CHAPTER 8 Conclusions $\quad 73$

INDEX 


\section{1}

\section{Introduction}

The principal example of a linear noisy network, and the one of greatest practical importance in electrical engineering, is a linear noisy amplifier. The noise performance of such amplifiers involves many questions of interest. One very significant question is the extent to which the amplifier influences signal-to-noise ratio over a narrow band (essentially at one frequency) in the system of which it is a part. We shall address ourselves exclusively to this feature, without intending to suggest that other features of the much larger noise-and-information problem are less important. The term "spot-noise performance" or merely "noise performance" will be used to refer to the effect of the amplifier upon the single-frequency signal-to-noise ratio. It is essential to emphasize right at the beginning the very restricted meaning these terms will have in our discussions.

We undertook the study reported here in the hope of formulating a rational approach to the characterization of amplifier spot-noise performance, and to its optimization by external circuit operations upon the terminals. Fortunately, a characterization has resulted which is based on a single hypothesis about the essential function of an amplifier and which turns out to avoid pitfalls previously associated with the effect of feedback upon noise performance. In developing the aforementioned noise characterization of amplifiers and in pursuing the relevant optimization problem, we encountered a number of illuminating features relating power and noise in linear multi-terminal-pair networks. Indeed, it eventually became clear that the major issues could be presented most simply by postponing until last the questions we had originally asked first. The result is a work of broader scope than was originally envisaged, 
and one for which the title "Circuit Theory of Linear Noisy Networks" seems appropriate.

Since the introduction by Friis ${ }^{1}$ and Fränz $z^{2}$ of the concept of spotnoise figure $F$ for the description of amplifier noise performance, this figure has played an essential role in communication practice. The noise figure is, however, merely a man-made definition, rather than a quantity deduced from clearly defined postulates or laws of nature. The possible consequences of this fact were never questioned deeply, although it has always been known that the (spot-) noise figure $F$ does not constitute a single absolute measure of amplifier noise performance.

In particular, the noise figure is a function of the impedance of the source connected to the amplifier input. Thus in giving an adequate conventional description of amplifier noise performance, the source impedance, as well as the noise figure, must be specified.

Usually, when regarded as a function of source impedance alone, the noise figure has a minimum value for some particular choice of this impedance. If with this source impedance the gain of a given amplifier remains sufficiently high, its noise figure will prescribe the noise figure of any amplifier cascade in which it is used as the first stage. In this way, it is possible to build an amplifier cascade with any desired high gain, and with a noise figure set by the minimum (with respect to source impedance) of the noise figure of the original amplifier.

If a cascade is to be composed of several individual amplifiers, each of which alone has a "high enough" gain when driven from the source impedance that yields its minimum noise figure, the previous argument shows that the amplifier with the lowest minimum noise figure should be used as the first stage. Any other choice would result in a higher over-all noise figure for the cascade.

The foregoing discussion seems to suggest that the minimum value (with respect to source impedance) of the noise figure of an amplifier may be used as an absolute measure of its noise performance and as a basis for comparison with other amplifiers. The validity of the argument, however, is based upon the two previously mentioned restrictions:

1. Each stage has "high enough" gain when driven from the "optimum" source that yields the minimum noise figure.

2. Only the source impedance of each stage is varied in controlling the noise performance.

The inadequacy of this viewpoint becomes clear when stage variables other than source impedance and stage interconnections other than the

${ }^{1}$ H. T. Friis, "Noise Figure of Radio Receivers," Proc. I.R.E., 32, 419 (1944).

${ }^{2} \mathrm{~K}$. Fränz, "Messung der Empfängerempfindlichkeit bei kurzen elektrischen Wellen," Z. Elektr. Elektroak., 59, 105 (1942). 
simple cascade become important in amplifier applications. The question of the quality of noise performance then becomes much more complicated. For example, when degenerative feedback is applied to an amplifier, its noise figure can be reduced to as close to unity as desired (for example, bypassing the entire amplifier with short circuits yields unit noise figure). But its gain is also reduced in the process. Indeed, if identical stages with the feedback are cascaded to recover the original single-stage gain before feedback, the resulting noise figure of the cascade cannot be less than that of the original amplifier. ${ }^{3}$ Moreover, with degenerative feedback the gain may easily be so greatly reduced that, as a first stage in a cascade, this amplifier alone no longer determines the over-all noise figure of the cascade. The minimum-noise-figure criterion considered above as a measure of amplifier noise performance breaks down. It appears that an absolute measure of amplifier noise performance must include, in addition to the specification of noise figure and source impedance, at least the specification of the gain.

The foregoing reasoning led us to the investigation presented in this study. Taking our clues from the results previously found by Haus and Robinson $^{4}$ for microwave amplifiers, and the method of active-network description presented by Mason, ${ }^{5}$ we searched for a measure of amplifier noise performance that would not only include the gain explicitly, as discussed earlier, but could also be minimized by external circuitry in a nontrivial way. Moreover, we believed that the minimum thus obtained should be a quantity characteristic of the amplifier itself. It should, for example, be invariant under lossless feedback, a type of feedback that does not appear to change the essential "noisy" character of the amplifier because it certainly adds no noise and can always be removed again by a realizable inverse lossless operation.

The precise form of a suitable noise-performance criterion has actually been known for many years, although its deeper significance somehow escaped attention. Indeed, the most glaring example of the correct criterion arises from the familiar problem of cascading two (or more) low-gain amplifiers having different noise figures $F_{1}$ and $F_{2}$ and different available gains $G_{1}(>1)$ and $G_{2}(>1)$.

The question is: If the available gain and noise figure of each amplifier do not change when the order of cascading is reversed, which cascade order leads to the best noise performance for the pair? Usually, "best noise performance" has been taken to mean "lowest noise figure" for the

${ }^{3}$ A. van der Ziel, Noise, Prentice-Hall, New York (1954).

${ }^{4} \mathrm{H}$. A. Haus and F. N. H. Robinson, "The Minimum Noise Figure of Microwave Beam Amplifiers," Proc. I.R.E., 43, 981 (1955).

${ }^{5}$ S. J. Mason, "Power Gain in Feedback Amplifiers," Trans. IRE, Professional Group on Circuit Theory, CT-1, No. 2, 20 (1954). 
pair, though in view of the answer obtained on that basis, the criterion should have been viewed with a little suspicion. Thus if $F_{12}$ and $F_{21}$ are the respective noise figures of the cascade when amplifier No. 1 and amplifier No. 2 are placed first, we have

$$
\begin{aligned}
& F_{12}=F_{1}+\frac{F_{2}-1}{G_{1}} \\
& F_{21}=F_{2}+\frac{F_{1}-1}{G_{2}}
\end{aligned}
$$

The condition that $F_{12}$ be less than $F_{21}$ is

$$
F_{1}-F_{2}<\frac{F_{1}-1}{G_{2}}-\frac{F_{2}-1}{G_{1}}
$$

or

$$
\left(F_{1}-1\right)-\left(F_{2}-1\right)<\frac{F_{1}-1}{G_{2}}-\frac{F_{2}-1}{G_{1}}
$$

or

$$
\frac{F_{1}-1}{1-\frac{1}{G_{1}}}<\frac{F_{2}-1}{1-\frac{1}{G_{2}}}
$$

That is, amplifier No. 1 should come first if Eq. 1.2 is satisfied.

Equation 1.2 implies that in a cascaded system of amplifiers, where the earliest stages are obviously the most critical in regard to noise performance, the "best" amplifier is the one having the lowest value not of $F$ but of the quantity

$$
M=\frac{F-1}{1-\frac{1}{G}}
$$

It is with $M$ that we shall be most concerned, and we shall call it the Noise Measure of an amplifier.

In terms of $M$, and the fact that the available gain of a cascaded pair of amplifiers is $G=G_{1} G_{2}, \mathrm{Eq} .1 .1 a$ becomes

$$
M_{12}=M_{1}+\frac{\Delta M}{G_{1}}\left(\frac{1-\frac{1}{G_{2}}}{1-\frac{1}{G}}\right)=M_{1}+\Delta M\left(\frac{G_{2}-1}{G-1}\right)
$$


where $\Delta M \equiv M_{2}-M_{1}$ is the difference between the noise measures of the second and first amplifiers of the cascade. Equation 1.4 shows that as long as $G_{1}$ and $G_{2}$ are greater than 1 the noise measure of a cascade of two amplifiers lies between the noise measures of its component amplifiers. In the particular case when the noise measures of the two amplifiers are equal, the resulting noise measure of the cascade is that of either amplifier, even if the available gains of the individual amplifiers are different.

Furthermore, since the available gain $G=G_{1} G_{2}$ is supposed to remain the same for either order of cascading, the result (Eq. 1.2) and the definition (Eq. 1.3) show that the lowest noise measure for a cascaded pair of amplifiers results from placing at the input the amplifier with the lowest individual noise measure.

Compared with the noise figure alone, which always deteriorates in a cascade (Eqs. 1.1) and which does not suffice to determine which amplifier should come first, the noise measure alone is evidently a more satisfactory and self-consistent single criterion of amplifier noise performance. Moreover, since noise measure and noise figure become essentially the same for amplifiers with sufficiently high gain, the final performance evaluation of a practical multistage amplifier always rests numerically (if not in principle) upon the familiar noise-figure criterion.

From such reasoning, we evolved a criterion for amplifier noise performance. The criterion is based on the plausible premise that, basically, amplifiers are supposed to provide "gain building blocks" without adding excessively to system noise. In its final stage of evolution, the criterion can be described as follows.

Suppose that $n$ different types of amplifiers are compared. An unlimited number of amplifiers of each type is assumed to be available. A general lossless (possibly nonreciprocal) interconnection of an arbitrary number of amplifiers of each type is then visualized, with terminals so arranged that in each case an over-all two-terminal-pair network is achieved. For each amplifier type, both the lossless interconnecting network and the number of amplifiers are varied in all possible ways to produce two conditions simultaneously:

1. A very high available gain (approaching infinity) for the over-all two-terminal-pair system when driven from a source having a positive real internal impedance.

2. An absolute minimum noise figure $F_{\min }$ for the resulting highgain system.

The value of $\left(F_{\min }-1\right)$ for the resulting high-gain two-terminal-pair network is taken specifically as the "measure of quality" of the amplifier type in each case. The "best" amplifier type will be the one yielding the smallest value of $\left(F_{\min }-1\right)$ at very high gain. 
The proof of this criterion will be developed through the concept of noise measure. Inasmuch as the general criterion involves (at least) arbitrary lossless interconnections of amplifiers, including feedback, input mismatch, and so forth, a rather general approach to the noise measure is required. In particular, we must show that the noise measure has a real significance of its own which is quite different from and much deeper than the one suggested by its appearance in Eq. 1.2. There it appears only as an algebraic combination of noise figure and available gain that happens to be convenient for describing amplifier cascades. Here the properties of $M$ with regard to lossless transformations are becoming involved.

Consideration of these properties brings us into the entire general subject of external network transformations of noisy linear networks. Among these, lossless transformations form a group in the mathematical sense. The quantities invariant under the group transformations must have a physical significance. Investigation of these invariants forms a substantial part of the present study. To be sure, for the special case of a two-terminal-pair amplifier, the optimum noise performance, through its related noise measure, turns out to be one of the invariants; but several other interpretations of the invariants prove equally interesting, and the development of the entire subject is simplified by presenting them first.

The simplest formulation and interpretation of the invariants of a linear noisy network result from its impedance representation. The following chapter is therefore devoted to a discussion of network transformations, or "imbeddings," in terms of the impedance-matrix representation. The concept of exchangeable power as an extension of available power is then introduced.

In Chapter 3 , the $n$ invariants of a linear noisy $n$-terminal-pair network are found as extrema of its exchangeable power, with respect to variations of a lossless $n$-to-one-terminal-pair network transformation. It is found that an $n$-terminal-pair network possesses not more than these $n$ invariants with respect to lossless $n$-to- $n$-terminal-pair transformations. These $n$ invariants are then exhibited in a particularly appealing way in the canonical form of the network, achievable by lossless transformations and characterized by exactly $n$ parameters. This form is introduced in Chapter 4.

Through Chapter 4, the invariants are interpreted only in terms of the extrema of the exchangeable power. New interpretations are considered next. They are best introduced by using other than the impedancematrix description. Accordingly, in Chapter 5, general matrix representations are studied, where it is pointed out that usually a different 
matrix description leads to a different interpretation of the invariants. In the case of an active two-terminal-pair network, a particularly important interpretation of the invariants is brought out by the generalcircuit-parameter-matrix description. This interpretation relates directly to the optimum "noise measure" of the network used as an amplifier and, therefore, to the minimum noise figure of the amplifier at arbitrarily high gain. Chapter 6 is devoted to this noise-measure concept and to the range of values that the noise measure may assume for a two-terminalpair amplifier subjected to arbitrary passive network transformations. In particular, the minimum value of the noise measure of the amplifier is found to be directly proportional to one of the two invariants of the amplifier.

A study is made of those arbitrary passive interconnections of twoterminal-pair amplifiers which result in an over-all two-terminal-pair amplifier. The conclusion is that the noise measure of the composite amplifier cannot be smaller than the optimum noise measure of the best component amplifier, namely, the amplifier with the smallest optimum noise measure.

The general theorems having established the existence of an optimum value of the noise measure of amplifiers, it remains in Chapter 7 to discuss in detail the network realization of this optimum for two-terminal-pair amplifiers. Some practical ways of achieving it are presented. Among these, the realization of optimum noise performance for a maser may be of greatest current interest.

With proof of the existence and realizability of a lower limit on the noise measure, and therefore of the noise figure at high gain, the major objective of the present work is accomplished. It is demonstrated that the quality with regard to noise performance of a two-terminal-pair amplifier can be specified in terms of a single number that includes the gain and that applies adequately to low-gain amplifiers.

We have previously published various separate discussions of some of these topics in different contexts. ${ }^{6-10}$ Each of these discussions has suffered from unnecessary complications because space limitations forced

${ }^{6}$ H. A. Haus and R. B. Adler, "Invariants of Linear Networks," 1956 IRE Convention Record, Part 2, 53 (1956).

${ }^{7}$ H. A. Haus and R. B. Adler, "Limitations des performances de bruit des amplificateurs linéaires," L'Onde Electrique, 38, 380 (1958).

${ }^{8}$ H. A. Haus and R. B. Adler, "Optimum Noise Performance of Linear Amplifiers," Proc, I.R.E., 46, 1517 (1958).

${ }^{9}$ R. B. Adler and H. A. Haus, "Network Realization of Optimum Amplifier Noise Performance," IRE Trans. on Circuit Theory, CT-5, No. 3, 156 (1958).

${ }^{10}$ H. A. Haus and R. B. Adler, "Canonical Form of Linear Noisy Networks," IRE Trans. on Circuit Theory, CT-5, No. 3, 161 (1958). 
them to be divorced from each other. It seemed, therefore, desirable to present the entire picture at greater leisure, particularly because the mathematical and logical complexity of the whole subject is thereby actually reduced. 


\section{Linear Noisy Networks in the Impedance Representation}

The discussion of the invariants of a linear noisy network under the group of lossless transformations is most simply carried out by using the impedance description of the network. We shall start by describing the effect of a lossless transformation on a general $n$-terminal-pair network. Such a network has to be classified with respect to its passiveactive character, which depends upon its ability to deliver or absorb power. The examination of power delivered or absorbed by a multiterminal-pair network raises some interesting questions leading to a generalization of the available-power concept: the exchangeable power. In Chap. 3, the exchangeable-power concept in turn leads to the discovery of the invariants mentioned in Chap. 1.

\subsection{Impedance-Matrix Representation of Linear Networks}

At any frequency, a linear $n$-terminal-pair network containing internal prescribed signal or noise generators is specified completely with respect to its terminal pairs by its impedance matrix $\mathbf{Z}$ and the complex Fourier amplitudes of its open-circuit terminal voltages $E_{1}, E_{2}, \cdots, E_{n}$ (Fig. 2.1). In matrix form, $\mathbf{Z}$ denotes a square $n$-by- $n$ array

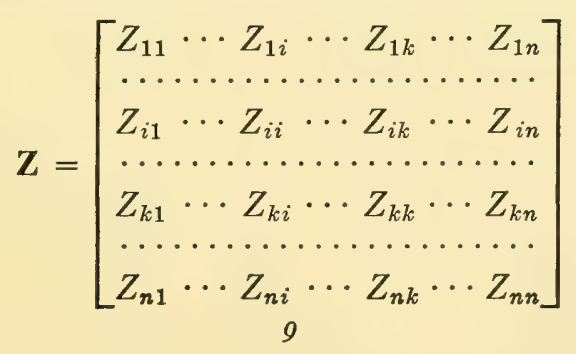




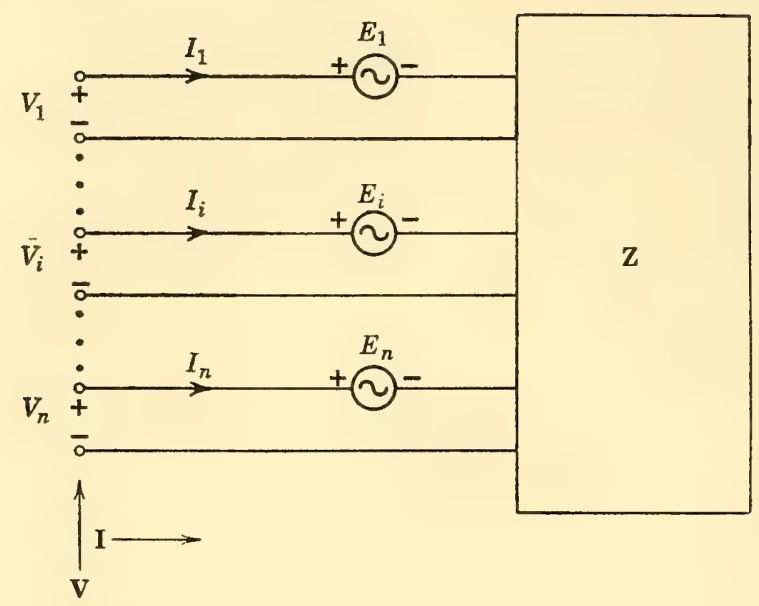

Fig. 2.1. Equivalent representation of linear network with internal noise sources.

The cases in which the $\mathbf{Z}$ matrix does not exist in a formal sense can be handled by suitable perturbations, for example, slight frequency changes or the addition of perturbing circuit elements. The complex amplitudes of the open-circuit terminal voltages are represented conveniently by a column matrix $\mathbf{E}$ :

$$
\mathbf{E}=\left[\begin{array}{c}
E_{1} \\
\cdot \\
\cdot \\
E_{i} \\
\cdot \\
\cdot \\
E_{k} \\
\cdot \\
\cdot \\
E_{n}
\end{array}\right]
$$

If the internal generators are random-noise sources, the Fourier amplitudes $E_{1} \cdots E_{n}$ are complex random variables, the physical significance of which usually appears in their self- and cross-power spectral densities $\overline{E_{i} E_{k}{ }^{*}{ }^{1}}$ The bar indicates an average over an ensemble of noise processes

${ }^{1}$ W. B. Davenport and W. O. Root, Random Signals and Noise, McGraw-Hill Book Company, New York (1958). 
with identical statistical properties. Here, as in the rest of this work, we retain only positive frequencies. In order to preserve the same multiplicative factors in power expressions for both random and nonrandom variables, we shall depart from convention by using root-mean-square values for all nonrandom complex amplitudes.

A convenient summary of the power spectral densities is the matrix

$$
\overline{\mathbf{E E}^{\dagger}}=\left[\begin{array}{l}
\overline{E_{1} E_{1}{ }^{*}} \ldots \overline{E_{1} E_{i}{ }^{*}} \ldots \overline{E_{1} E_{k}{ }^{*}} \ldots \overline{E_{1} E_{n}{ }^{*}} \\
\cdots \cdots \cdots \\
\overline{E_{i} E_{1}{ }^{*}} \ldots \overline{E_{i} E_{i}{ }^{*}} \ldots \overline{E_{i} E_{k}{ }^{*}} \ldots \overline{E_{i} E_{n}{ }^{*}} \\
\cdots \cdots \\
\overline{E_{k} E_{1}{ }^{*}} \ldots \overline{E_{k} E_{i}{ }^{*}} \ldots \overline{E_{k} E_{k}{ }^{*}} \ldots \overline{E_{k} E_{n}{ }^{*}} \\
\cdots \cdots \cdots \\
\overline{E_{n} E_{1}{ }^{*}} \ldots \overline{E_{n} E_{i}{ }^{*}} \ldots \overline{E_{n} E_{k}{ }^{*}} \cdots \overline{E_{n} E_{n}{ }^{*}}
\end{array}\right]
$$

where the superscript dagger indicates the two-step operation composed of forming the complex conjugate of and transposing the matrix to which it refers. Briefly, $\mathbf{A}^{\dagger}$ is called the Hermitian conjugate of any matrix $\mathbf{A .}^{2}$ The matrix $\overline{\mathbf{E E}^{\dagger}}$ is its own Hermitian conjugate, because $\overline{E_{i} E_{k}{ }^{*}}=$ $\overline{E_{k}{ }^{*} E_{i}}=\left(\overline{E_{k} E_{i}^{*}}\right)^{*}$. Such a matrix is said to be Hermitian.

In addition, we can show that $\overline{\mathbf{E E}}$ is a positive definite or semidefinite matrix. Construct the real nonnegative quadratic form:

$\overline{\left(x_{1}^{*} E_{1}+\ldots+x_{i}^{*} E_{i}+\ldots+x_{n}{ }^{*} E_{n}\right)\left(x_{1} E_{1}^{*}+\ldots+x_{i} E_{i}^{*}+\ldots+x_{n} E_{n}^{*}\right)} \geq 0$

where the $x_{i}$ are arbitrary complex numbers. In terms of the column matrix

$$
\mathbf{x}=\left[\begin{array}{c}
x_{1} \\
\cdot \\
\cdot \\
x_{i} \\
\cdot \\
\cdot \\
x_{n}
\end{array}\right]
$$

and the column matrix $\mathbf{E}$, the foregoing expression becomes

$$
\overline{\mathbf{x}^{\dagger} \mathbf{E}\left(\mathbf{x}^{\dagger} \mathbf{E}\right)^{\dagger}}=\mathbf{x}^{\dagger} \overline{\mathbf{E E}^{\dagger}} \mathbf{x} \geq 0
$$

${ }^{2}$ F. B. Hildebrand, Methods of Applied Mathematics, Prentice-Hall, New York (1952). 
for all $\mathbf{x}$. If the inequality sign applies for all $\mathbf{x} \neq \mathbf{0}, \overline{\mathbf{E E}^{\dagger}}$ is positive definite; if the equality sign applies for some (but not all) $\mathbf{x} \neq \mathbf{0}, \overline{\mathbf{E E}}{ }^{\dagger}$ is positive semidefinite. The equality sign for all $\mathbf{x} \neq \mathbf{0}$ would occur only in the trivial case $\mathbf{E}=\mathbf{0}$.

\subsection{Lossless Transformations}

If the $n$-terminal-pair network with the generator column matrix $\mathbf{E}$ and the impedance matrix $\mathbf{Z}$ is connected properly to a $2 n$-terminal-pair network, a new $n$-terminal-pair network may be obtained. It will have a new noise column matrix $\mathbf{E}^{\prime}$ and a new impedance matrix $\mathbf{Z}^{\prime}$. This

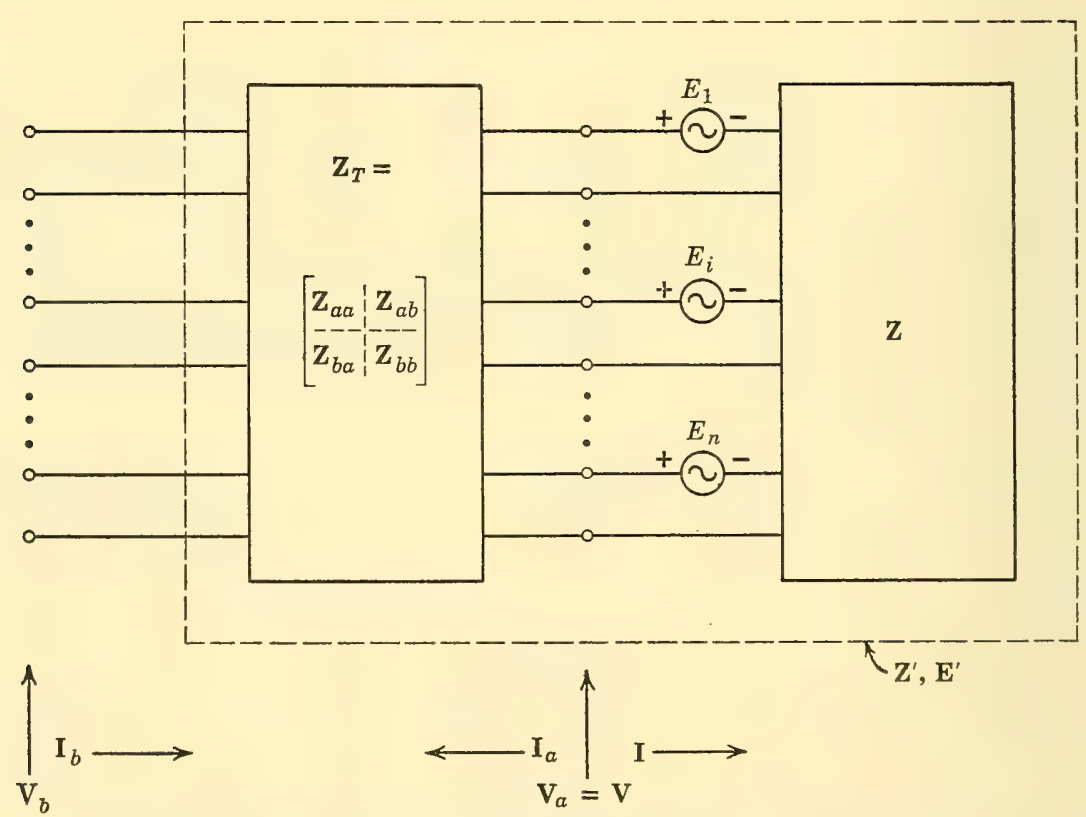

Fig. 2.2. General transformation (imbedding) of an $n$-terminal-pair network.

operation, shown in Fig. 2.2, we shall call a transformation, or an imbedding, of the original network. If the added network is lossless, this arrangement represents the most general lossless modification (including the addition of multiple, and not necessarily reciprocal, feedback paths) that can be performed externally upon the original $n$-terminal-pair network so as to obtain a new $n$-terminal-pair network.

The analytical relation between the voltages and currents applied to the $2 n$-terminal-pair, lossless network (the "transformation network") 
of Fig. 2.2 can be written in the form

$$
\begin{aligned}
& \mathbf{V}_{a}=\mathbf{Z}_{a a} \mathbf{I}_{a}+\mathbf{Z}_{a b} \mathbf{I}_{b} \\
& \mathbf{V}_{b}=\mathbf{Z}_{b a} \mathbf{I}_{a}+\mathbf{Z}_{b b} \mathbf{I}_{b}
\end{aligned}
$$

The column vectors $\mathbf{V}_{a}$ and $\mathbf{V}_{b}$ comprise the terminal voltages applied to the transformation network on its two sides, and the column vectors $\mathbf{I}_{a}$ and $\mathbf{I}_{b}$ comprise the currents $\mathbf{I}_{T}$ flowing into it. The four $\mathbf{Z}$ matrices in Eqs. 2.2 and 2.3 are each square and of $n$th order. They make up the square $2 n$ th-order matrix $\mathbf{Z}_{T}$ of the lossless transformation network. The condition of losslessness can be summarized in the following relations, which express the fact that the total time-average power $P$ into the transformation network must be zero for all choices of the terminal currents:

$$
P=\frac{\mathbf{1}}{4} \mathbf{I}_{T}^{\dagger}\left(\mathbf{Z}_{T}+\mathbf{Z}_{T}^{\dagger}\right) \mathbf{I}_{T} \equiv 0, \quad \text { for all } \mathbf{I}_{T}
$$

therefore

$$
\mathbf{z}_{T}+\mathbf{z}_{T}^{\dagger}=0
$$

or

$$
\mathbf{Z}_{a a}=-\mathbf{Z}_{a a}{ }^{\dagger} ; \quad \mathbf{Z}_{a b}=-\mathbf{Z}_{b a}{ }^{\dagger} ; \quad \mathbf{Z}_{b b}=-\mathbf{Z}_{b b}{ }^{\dagger}
$$

Equation 2.5 does not require that the transformation network be reciprocal.

The original $n$-terminal-pair network, with impedance matrix $\mathbf{Z}$ and noise column matrix $\mathbf{E}$, imposes the following relations between the column matrices $\mathbf{V}$ and $\mathbf{I}$ of the voltages across, and the currents into, its terminals:

$$
\mathbf{V}=\mathbf{Z I}+\mathbf{E}
$$

The currents I into the $n$-terminal-pair network are, according to Fig. 2.2, equal and opposite to the currents $\mathbf{I}_{a}$ into one side of the $2 n$-terminal-pair network. The voltages $\mathbf{V}$ are equal to the voltages $\mathbf{V}_{a}$. We thus have

$$
\mathbf{V}=\mathbf{V}_{a} ; \quad \mathbf{I}=-\mathbf{I}_{a}
$$

Introduction of Eqs. 2.7 into Eq. 2.6 and application of the latter to Eq. 2.2 give

$$
\mathbf{I}_{a}=-\left(\mathbf{Z}+\mathbf{Z}_{a a}\right)^{-1} \mathbf{Z}_{a b} \mathbf{I}_{b}+\left(\mathbf{Z}+\mathbf{Z}_{a a}\right)^{-1} \mathbf{E}
$$

When this equation is substituted in Eq. 2.3, the final relation between $\mathbf{V}_{b}$ and $\mathbf{I}_{b}$ is determined:

where

$$
\mathbf{V}_{b}=\mathbf{Z}^{\prime} \mathbf{I}_{b}+\mathbf{E}^{\prime}
$$

$$
\mathbf{Z}^{\prime}=-\mathbf{Z}_{b a}\left(\mathbf{Z}+\mathbf{Z}_{a a}\right)^{-1} \mathbf{Z}_{a b}+\mathbf{Z}_{b b}
$$


and

$$
\mathbf{E}^{\prime}=\mathbf{Z}_{b a}\left(\mathbf{Z}+\mathbf{Z}_{a a}\right)^{-1} \mathbf{E}
$$

Equation 2.8 is the matrix relation for the new $n$-terminal-pair network obtained from the original one by imbedding it in a $2 n$-terminal-pair network. Here $\mathbf{Z}^{\prime}$ is the new impedance matrix, and $\mathbf{E}^{\prime}$ is the column matrix of the new open-circuit noise voltages. Conditions 2.5 must be applied to Eqs. 2.9 and 2.10 if the transformation network is to be lossless.

\subsection{Network Classification in Terms of Power}

In the course of our general study of noise performance of linear amplifiers, it will be necessary to generalize the definition of available power. The need arises from situations involving negative resistance.

Normally, the available power $P_{\mathrm{av}}$ of a one-terminal-pair source is defined as:

$P_{\mathrm{av}} \equiv$ the greatest power that can be drawn from the source by arbitrary variation of its terminal current (or voltage)

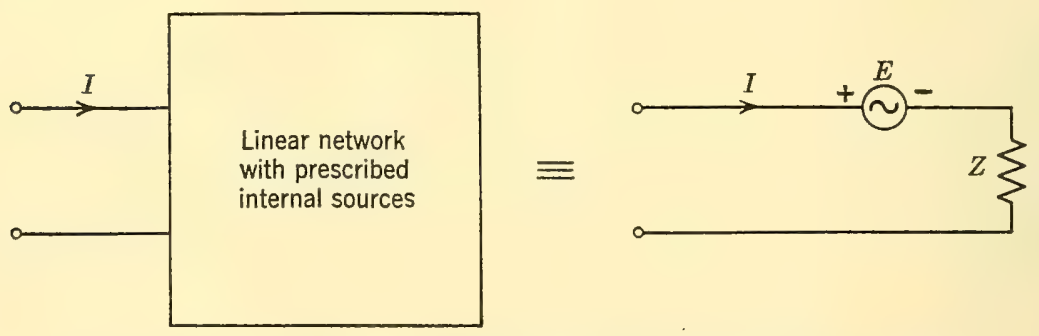

Fig. 2.3. The Thévenin equivalent of a one-terminal-pair linear network.

If the Thévenin representation of the source (Fig. 2.3) has the complex open-circuit voltage $E$ and internal impedance $Z$, with $R=$ $\operatorname{Re}(Z)>0$, this definition leads to

$$
P_{\mathrm{av}}=\frac{1}{4} \frac{\overline{E E^{*}}}{R}=\frac{1}{2} \frac{\overline{E E^{*}}}{Z+Z^{*}}>0, \quad \text { for } R>0
$$

If the source is nonrandom, the bar in Eq. $2.11 a$ may be omitted. No other changes are necessary because $E$ is then understood to be a rootmean-square amplitude, as remarked in Sec. 2.1. In Eq. 2.11a, $P_{\mathrm{av}}$ is also a stationary value (extremum) of the power output regarded as a function of the complex terminal current $I$. Moreover, the available power (Eq. 2.11a) can actually be delivered to the (passive) load $Z^{*}$. 
When $R$ is negative, however, the foregoing definition of available power leads to

$$
P_{\mathrm{av}}=\infty, \quad \text { for } R<0
$$

since this is indeed the greatest power obtainable from such a source, and is achievable by loading it with the (passive) impedance $-Z$. Observe that this result is not either a stationary value or extremum of the power output as a function of terminal current, nor is it consistent with Eq. 2.11 $a$ extended to negative values of $R$.

To retain the stationary property (with respect to terminal current) of the normal available power concept, and accordingly to preserve the form of Eq. 2.11a, we define the concept of exchangeable power $P_{e}$ :

$$
\begin{aligned}
P_{e} \equiv & \text { the stationary value (extremum) of the power output from the } \\
& \text { source, obtained by arbitrary variation of the terminal current } \\
& \text { (or voltage) }
\end{aligned}
$$

It is easy to show that this definition of exchangeable power always leads to Eq. $2.11 a$ for any nonzero value of $R$ in Fig. 2.3. Specifically,

$$
P_{e}=\frac{1}{4} \frac{\overline{E E^{*}}}{R}=\frac{1}{2} \frac{\overline{E E^{*}}}{Z+Z^{*}}, \quad \text { for } R \neq 0
$$

When $R$ is negative, $P_{e}$ in Eq. $2.11 b$ is negative. Its magnitude then represents the maximum power that can be pushed into the terminals by suitable choice of the complex terminal current $I$. This situation may also be realized by connecting the (nonpassive) conjugate-match impedance $Z^{*}$ to the terminals. This impedance actually functions as a source, pushing the largest possible power into the network terminals.

A straightforward extension of the exchangeable-power definition to $n$-terminal-pair networks makes it the stationary value (extremum) of the total power output from all the terminal pairs, obtained by arbitrary variations of all the terminal currents. With reference to Fig. 2.1, we search specifically for the stationary values with respect to $\mathbf{I}$ of the total average output power $P$ of the network

$$
P=-\frac{1}{2} \overline{\left[\mathbf{I}^{\dagger}\left(\mathbf{Z}+\mathbf{Z}^{\dagger}\right) \mathbf{I}+\mathbf{I}^{\dagger} \mathbf{E}+\mathbf{E}^{\dagger} \mathbf{I}\right]}
$$

In Eq. 2.12, $P$ is a quadratic function of the terminal currents. The stationary values of interest depend upon a particular classification of the impedance matrix $\mathbf{Z}$. This classification is based upon Eq. 2.12, with the internal sources inactive.

With the internal generators inactive, $\mathbf{E}$ becomes zero, and the power 
leaving the network is

$$
P_{o}=-\frac{1}{2} \overline{\left[\mathbf{I}^{\dagger}\left(\mathbf{Z}+\mathbf{Z}^{\dagger}\right) \mathbf{I}\right]}
$$

Three possible cases have to be distinguished for $P_{o}$ (aside from the previously discussed case of a lossless network).

1. The first case is that of a passive network. Then the power output $P_{o}$ must not be positive for any $\mathbf{I}$, indicating a net (or zero) absorption of power inside the network. The matrix $\left(\mathbf{Z}+\mathbf{Z}^{\dagger}\right)$ is positive (semi) definite.

2. In the second case, the matrix $\left(\mathbf{Z}+\mathbf{Z}^{\dagger}\right)$ is negative (semi) definite, which means that the power $P_{o}$ flowing out of the network is never negative, regardless of the terminal currents $\mathbf{I}$. This indicates a net (or zero) generation of power inside the network.

3. Finally, the matrix $\left(\mathbf{Z}+\mathbf{Z}^{\dagger}\right)$ may be indefinite. The power $P_{o}$ flowing out of the network may be either positive or negative, depending upon the currents $\mathbf{I}$.

One may imagine the power $P_{o}$ plotted in the multidimensional space of the complex current amplitudes $\mathbf{I}$. The three cases may be distinguished according to the nature of the quadratic surface $P_{o}$. When $\left(\mathbf{Z}+\mathbf{Z}^{\dagger}\right)$ is either positive or negative semidefinite, the surface is a multidimensional paraboloid with a maximum or minimum, respectively, at the origin. When $\left(\mathbf{Z}+\mathbf{Z}^{\dagger}\right)$ is indefinite, the surface is a hyperboloid with a stationary point (saddle point) at the origin. The word "origin" is used loosely for simplicity; it omits the semidefinite cases, when $\left(\mathbf{Z}+\mathbf{Z}^{\dagger}\right)$ is singular. Then difficulties will arise in connection with the inverse of $\left(\mathbf{Z}+\mathbf{Z}^{\dagger}\right)$. These difficulties will be circumvented by the addition of suitable small loss, in order to remove the singularity. Results pertaining to the singular case can be obtained in the limit of vanishing added loss. Henceforth we shall make no explicit reference to semidefinite cases.

The power $P$ out of the network in the presence of internal generators is obtained from $P_{o}$ (Eq. 2.12) by adding to it a plane through the origin. The extremum or saddle point ceases to occur at the origin. The new position of the stationary point can be determined conveniently by introducing an appropriate shift of coordinates. Setting

$$
\mathbf{I}^{\prime}=\mathbf{I}+\left(\mathbf{Z}+\mathbf{Z}^{\dagger}\right)^{-1} \mathbf{E}
$$

yields for Eq. 2.12

$$
P=-\frac{1}{2} \overline{\left[\left(\mathbf{I}^{\prime}\right)^{\dagger}\left(\mathbf{Z}+\mathbf{Z}^{\dagger}\right)\right.} \overline{\left.\mathbf{I}^{\prime}-\mathbf{E}^{\dagger}\left(\mathbf{Z}+\mathbf{Z}^{\dagger}\right)^{-1} \mathbf{E}\right]}
$$

The shift of origin has led to a completion of the square. The new origin 
is obviously the stationary point of the power expressed in the new variables $\mathbf{I}^{\prime}$. The height of the surface at the stationary point is the exchangeable power $P_{e}$ :

$$
P_{e}=\frac{1}{2} \overline{\left[\mathbf{E}^{\dagger}\left(\mathbf{Z}+\mathbf{Z}^{\dagger}\right)^{-1} \mathbf{E}\right]}
$$

Because the definite characters of $\left(\mathbf{Z}+\mathbf{Z}^{\dagger}\right)^{-1}$ and $\left(\mathbf{Z}+\mathbf{Z}^{\dagger}\right)$ are the same: $P_{e} \geq 0$ (regardless of $\mathbf{E}$ ) when $\left(\mathbf{Z}+\mathbf{Z}^{\dagger}\right)$ is positive definite; $P_{e} \leq 0$ (regardless of $\mathbf{E}$ ) when $\left(\mathbf{Z}+\mathbf{Z}^{\dagger}\right)$ is negative definite and $P_{e} \geqslant 0$ (depending upon the particular $\mathbf{E}$ involved) when $\left(\mathbf{Z}+\mathbf{Z}^{\dagger}\right)$ is indefinite. In view of the term $\left(\mathbf{I}^{\prime}\right)^{\dagger}\left(\mathbf{Z}+\mathbf{Z}^{\dagger}\right) \mathbf{I}^{\prime}$ in $P$, the significance of $P_{e}$ in Eq. 2.15 is of the same kind as that of $P_{e}$ in Eq. $2.11 b$ when $\left(\mathbf{Z}+\mathbf{Z}^{\dagger}\right)$ is either positive or negative definite. When $\left(\mathbf{Z}+\mathbf{Z}^{\dagger}\right)$ is indefinite, however, $P_{e}$ in Eq. 2.15 is simply the stationary-point (saddle-point) value of the average output power with respect to variations of the terminal currents, and has no analog in the case of a one-terminal-pair network (Eq. 2.11b).

We have defined the exchangeable power for a one-terminal-pair network as the extremum of power output obtainable by arbitrary variation of terminal current. In an obvious generalization, we have extended this definition to $n$-terminal-pair networks by considering the extremum of the power output of the network obtained by an arbitrary variation of all its terminal currents. In this case, we have encountered the possibility of the output power assuming a stationary value rather than an extremum. One may ask whether the stationary value of the output power for the multiterminal-pair case could be achieved in a simpler way. One obvious method to try is that shown in Fig. $2.4 .^{3}$

The given network is imbedded in a variable $(n+1)$-terminal-pair lossless network. For each choice of the variable lossless network, we consider first the power that can be drawn from the $(n+1)$ th pair for various values of the complex current $I_{n+1}$ (that is, for various "loadings" of this terminal pair). In particular then, we determine the exchangeable power $P_{e, n+1}$ for this terminal pair according to Eq. $2.11 b$, recognizing that it may be either positive or negative. In the respective cases, its magnitude represents power delivered by, or to, the original network, since the imbedding network is lossless. Specifically, its magnitude represents the greatest possible value of the power that can be drawn from, or delivered to, the original network, for a given choice of the lossless imbedding network.

${ }^{3}$ Recently we have learned that, prior to our study, this particular noisy-network power-optimization problem was considered and solved independently for receiving antennas by J. Granlund, Topics in the Design of Antennas for Scatter, M.I.T. Lincoln Laboratory Technical Report 135, Massachusetts Institute of Technology, Cambridge, Mass. (1956). 
The exchangeable power determined in this way will, in general, be different for different choices of the lossless network because the contributions of the $n$ terminal pairs of the original network are in each case combined with different relative magnitudes and phases to make up the output at terminals $n+1$. Therefore, we might expect the available power of the original $n$-terminal-pair network to be the extremum value of $P_{e, n+1}$ obtainable by considering every possible variation of the lossless

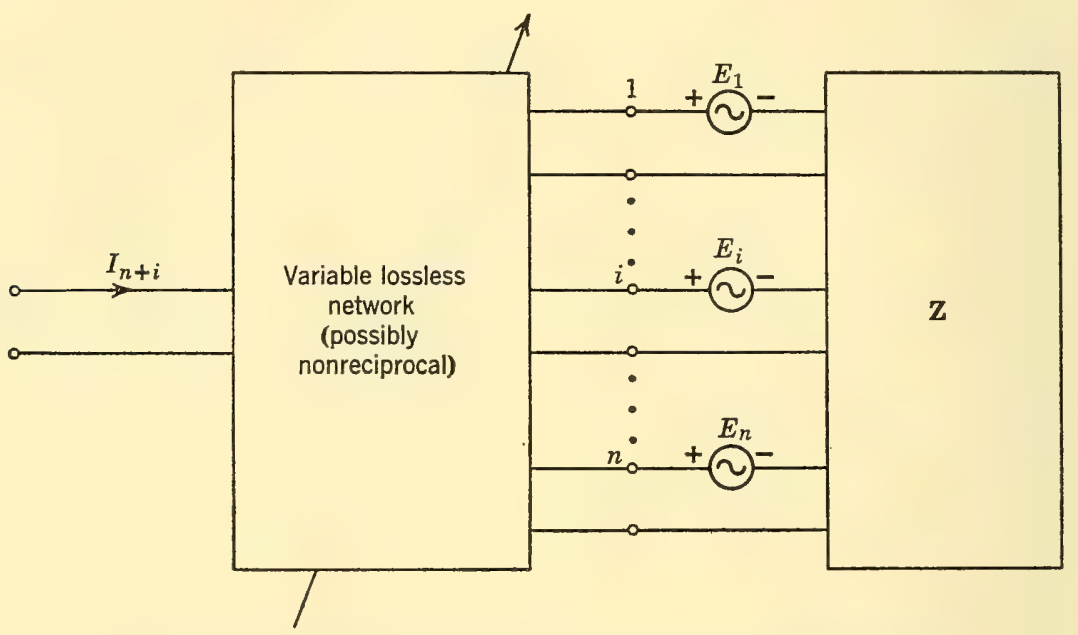

Fig, 2.4. Imbedding into an $(n+1)$-terminal-pair lossless network.

transformation network. As we shall see shortly, this result proves to be correct only in one simple case, namely, that in which the original $n$ terminal-pair network contains only coherent signal generators. In such a case $P_{e, n+1}$ has only one stationary value as the lossless network is varied, and this value is precisely the exchangeable power discussed previously ( $P_{e}$ in Eq. 2.15).

In the general situation of an arbitrary noisy network, we shall find that $P_{e, n+1}$ has $n$ stationary values as the lossless transformation network is varied in all possible ways. None of these individually is the exchangeable power for the original network. The sum of them, however, does prove to be the exchangeable power. The major burden of the discussion immediately to follow will be to interpret the stationary values of $P_{e, n+1}$ in terms of some physical properties of the original network. In later chapters, the relationship of these results with the noise performance will emerge. 


\section{Impedance Formulation}

\section{of the Characteristic-Noise Matrix}

We shall proceed to a close examination of the stationary-value problem posed in connection with Fig. 2.4, at the end of the last section and prove the assertions made about it. A matrix formulation of the problem will be required, which will reduce the problem to one in matrix eigenvalues. The corresponding eigenvalues are those of a new matrix, the "characteristic-noise matrix." Some general features of the eigenvalues will be studied, including their values for two interesting special cases. The effect of lossless imbeddings upon the eigenvalues will be discussed to complete the background for the noise-performance investigations.

\subsection{Matrix Formulation of Stationary-Value Problem}

The network operation indicated in Fig. 2.4 is conveniently accomplished by first imbedding the original $n$-terminal-pair network $\mathbf{Z}$ in a lossless $2 n$-terminal-pair network, as indicated in Fig. 2.2. Open-circuiting all terminal pairs of the resulting $n$-terminal-pair network $\mathbf{Z}^{\prime}$, except the $i$ th, we achieve the $n$-to-1-terminal-pair lossless transformation indicated in Fig. 2.4. The exchangeable power from the $i$ th terminal pair of the network $\mathbf{Z}^{\prime}$ can be written in matrix form as

$$
P_{e, i}=\frac{1}{2} \frac{\overline{E_{i}{ }^{\prime} E_{i}{ }^{*}}}{Z_{i i}{ }^{\prime}+Z_{i i}{ }^{\prime *}}=\frac{1}{2} \frac{\boldsymbol{\xi}^{\dagger} \overline{\mathbf{E}^{\prime} \mathbf{E}^{\prime \dagger} \xi}}{\boldsymbol{\xi}^{\dagger}\left(\mathbf{Z}^{\prime}+\mathbf{Z}^{\prime}\right)^{\dagger \xi}}
$$


where the (real) column matrix $\xi$ has every element zero except the $i$ th, which is 1 :

$$
\xi=\left[\begin{array}{c}
\xi_{1} \\
\cdot \\
\cdot \\
\xi_{i} \\
\cdot \\
\cdot \\
\cdot \\
\xi_{n}
\end{array}\right]=\left[\begin{array}{c}
0 \\
\cdot \\
\cdot \\
\cdot \\
1 \\
\cdot \\
\cdot \\
0
\end{array}\right], \quad\left\{\begin{array}{l}
\xi_{j}=0, \quad j \neq i \\
\xi_{i}=1
\end{array}\right.
$$

Matrix $\xi$ can be visualized as a double-pole $n$-throw selector switch which chooses electrically only one of the $n$ terminal pairs according to its sole nonzero element.

The variation of the lossless network in Fig. 2.4 now corresponds to variation of the transformation network $\mathbf{Z}_{T}$ in Fig. 2.2 through all possible lossless forms. We wish to find the stationary values of $P_{e, i}$ corresponding to variation of $\mathbf{Z}_{T}$. To render explicit this variation, $\mathbf{E}^{\prime}$ is first expressed in terms of the original $\mathbf{E}$ and $\mathbf{Z}$, using Eq. 2.10. Accordingly,

$$
\overline{\mathbf{E}^{\prime} \mathbf{E}^{\prime \dagger}}=\mathbf{Z}_{b a}\left(\mathbf{Z}+\mathbf{Z}_{a a}\right)^{-1} \overline{\mathbf{E \mathbf { E } ^ { \dagger }}}\left[\left(\mathbf{Z}+\mathbf{Z}_{a a}\right)^{-1}\right]^{\dagger} \mathbf{Z}_{b a}^{\dagger}=\boldsymbol{\tau}^{\dagger} \overline{\mathbf{E} \mathbf{E}^{\dagger} \boldsymbol{\tau}}
$$

where

$$
\boldsymbol{\tau}^{\dagger}=\mathbf{Z}_{b a}\left(\mathbf{Z}+\mathbf{Z}_{a a}\right)^{-1}
$$

Second, expressing $\mathbf{Z}^{\prime}$ in terms of $\mathbf{Z}$ by means of $\mathrm{Eq} .2 .9$ yields

$$
\begin{aligned}
\mathbf{Z}^{\prime}+\mathbf{Z}^{\prime \dagger}= & -\mathbf{Z}_{b a}\left(\mathbf{Z}+\mathbf{Z}_{a a}\right)^{-1} \mathbf{Z}_{a b}+\mathbf{Z}_{b b}+\mathbf{Z}_{b b}^{\dagger} \\
& -\mathbf{Z}_{a b}^{\dagger}\left[\left(\mathbf{Z}+\mathbf{Z}_{a a}\right)^{-1}\right]^{\dagger} \mathbf{Z}_{b a}^{\dagger}
\end{aligned}
$$

The conditions of Eq. 2.5, guaranteeing that the transformation network is lossless, convert the foregoing relation to

$$
\begin{aligned}
\mathbf{Z}^{\prime}+\mathbf{Z}^{\prime \dagger} & =\mathbf{Z}_{b a}\left[\left(\mathbf{Z}+\mathbf{Z}_{a a}\right)^{-1}+\left(\mathbf{Z}^{\dagger}+\mathbf{Z}_{a a}^{\dagger}\right)^{-1}\right] \mathbf{Z}_{b a}^{\dagger} \\
& =\mathbf{Z}_{b a}\left(\mathbf{Z}+\mathbf{Z}_{a a}\right)^{-1}\left[\left(\mathbf{Z}^{\dagger}+\mathbf{Z}_{a a}^{\dagger}\right)+\left(\mathbf{Z}+\mathbf{Z}_{a a}\right)\right]\left(\mathbf{Z}^{\dagger}+\mathbf{Z}_{a a}^{\dagger}\right)^{-1} \mathbf{Z}_{b a}{ }^{\dagger} \\
& =\boldsymbol{\tau}^{\dagger}\left(\mathbf{Z}+\mathbf{Z}^{\dagger}\right) \boldsymbol{\tau}
\end{aligned}
$$

It follows that

$$
P_{e, i}=\frac{1}{2} \frac{\left(\xi^{\dagger} \tau^{\dagger}\right) \overline{\mathbf{E E}^{\dagger}}(\boldsymbol{\tau} \xi)}{\left(\xi^{\dagger} \tau^{\dagger}\right)\left(\mathbf{Z}+\mathbf{Z}^{\dagger}\right)(\tau \xi)}
$$

in which matrix $\tau$ (not $\xi$ ) is to be varied through all possible values consistent with the lossless requirements upon the transformation network. 
The significant point now is that $\tau$ is actually any square matrix of order $n$ because $\mathbf{Z}_{b a}$ in Eq. 3.4 is entirely unrestricted! Therefore, a new column matrix $\mathbf{x}$ may be defined as

$$
\mathbf{x}=\tau \xi=\left[\begin{array}{c}
x_{1} \\
\cdot \\
\cdot \\
\cdot \\
x_{i} \\
\cdot \\
\cdot \\
\cdot \\
x_{n}
\end{array}\right]
$$

in which the elements take on all possible complex values as the lossless transformation network $\mathbf{Z}_{T}$ in Fig. 2.2 is varied through all its allowed forms. Consequently, the stationary values of $P_{e, i}$ in Eq. 3.7 may be found most conveniently by determining instead the stationary values of the (real) expression

$$
P_{e, i}=\frac{1}{2} \frac{\mathbf{x}^{\dagger} \overline{\mathbf{E E}^{\dagger}} \mathbf{x}}{\left.\mathbf{Z}+\mathbf{Z}^{\dagger}\right) \mathbf{x}}
$$

as the complex column matrix $\mathbf{x}$ is varied quite arbitrarily.

Aside from the uninteresting possibility of a lossless original network $\mathbf{Z}$, three cases must be distinguished in Eq. 3.9, corresponding to the three different characters of $\mathbf{Z}$ discussed previously in connection with Eqs. 2.13 and 2.15. Since $\overline{\mathbf{E E}}$ is positive (semi) definite, these cases are described as follows in terms of the variation of $P_{e, i}$ as a multidimensional function of all the complex components of $\mathbf{x}$ :

(a) $\mathbf{Z}+\mathbf{Z}^{\dagger}$ positive definite; $P_{e, i}>0$ for all $\mathbf{x}$.

(b) $\mathbf{Z}+\mathbf{Z}^{\dagger}$ negative definite; $P_{e, i}<0$ for all $\mathbf{x}$.

(c) $\mathbf{Z}+\mathbf{Z}^{\dagger}$ indefinite; $P_{e, i} \gtrless 0$, depending upon $\mathbf{x}$.

\subsection{Eigenvalue Formulation of Stationary-Value Problem}

We now turn to the determination of the extrema and stationary values of $P_{e, i}$ in Eq. 3.9. For reasons that will become clear in regard to amplifier noise performance, we shall look for extrema of the quantity $p=-P_{e, i}$. In terms of $\mathbf{A}=\overline{\mathbf{E E}^{\dagger}}$ and $\mathbf{B}=-2\left(\mathbf{Z}+\mathbf{Z}^{\dagger}\right)$,

$$
p=-\frac{\mathbf{x}^{\dagger} \overline{\mathbf{E E ^ { \dagger }} \mathbf{x}}}{2 \mathbf{x}^{\dagger}\left(\mathbf{Z}+\mathbf{Z}^{\dagger}\right) \mathbf{x}}=\frac{\mathbf{x}^{\dagger} \mathbf{A} \mathbf{x}}{\mathbf{x}^{\dagger} \mathbf{B} \mathbf{x}}
$$


The stationary conditions and corresponding values for $p$ may be obtained from the solution of the equivalent problem of determining the stationary values of $\mathbf{x}^{\dagger} \mathbf{B x}$, subject to the constraint $\mathbf{x}^{\dagger} \mathbf{A} \mathbf{x}=$ constant. Therefore, introduction of the Lagrange multiplier $1 / \lambda$ and recognition that $p$ may be regarded as a function of either the set of $x_{i}$ or the set of $x_{i}{ }^{*}$ lead to the conditions

$$
\frac{\partial}{\partial x_{i}{ }^{*}}\left(\mathbf{x}^{\dagger} \mathbf{B} \mathbf{x}-\frac{1}{\lambda} \mathbf{x}^{\dagger} \mathbf{A} \mathbf{x}\right)=0, \quad i=1,2, \cdots, n
$$

or simply

$$
\mathbf{A x}-\lambda \mathbf{B} \mathbf{x}=(\mathbf{A}-\lambda \mathbf{B}) \mathbf{x}=0
$$

The values of $\lambda$ are then fixed by the requirement

$$
\operatorname{det}(\mathbf{A}-\lambda \mathbf{B})=0=\operatorname{det}\left(\mathbf{B}^{-1} \mathbf{A}-\lambda \mathbf{I}\right)
$$

where $\mathbf{I}$ is the unit matrix. This means that the values of $\lambda$ are just the eigenvalues of the matrix $\mathbf{B}^{-1} \mathbf{A} \equiv-\frac{1}{2}\left(\mathbf{Z}+\mathbf{Z}^{\dagger}\right)^{-1} \overline{\mathbf{E} \mathbf{E}^{\dagger}}$.

The matrix $\mathbf{x}$ yielding any stationary point of $p$ must satisfy Eqs. 3.12, as well as the constraint $\mathbf{x}^{\dagger} \mathbf{A x}=$ constant. Let $\lambda_{s}$ be one eigenvalue of $\mathbf{B}^{-1} \mathbf{A}$ and $\mathbf{x}^{(s)}$ be the corresponding solution (eigenvector) of Eq. 3.12. Then premultiplication of Eq. 3.12 by $\mathbf{x}^{(s) \dagger}$ yields

$$
\mathbf{x}^{(s) \dagger} \mathbf{A} \mathbf{x}^{(s)}=\lambda_{s} \mathbf{x}^{(s) \dagger} \mathbf{B} \mathbf{x}^{(s)}
$$

or

$$
\lambda_{s}=\frac{\mathbf{x}^{(s) \dagger} \mathbf{A} \mathbf{x}^{(s)}}{\mathbf{x}^{(s) \dagger} \mathbf{B} \mathbf{x}^{(s)}}=p_{\text {stat }}
$$

which is real and equal to the corresponding stationary value of $p$.

It follows that the stationary values of the exchangeable power $P_{e, i}$ are the negatives of the (real) eigenvalues of the matrix

$$
\mathbf{A} \mathbf{B}^{-1}=-\frac{1}{2}\left(\mathbf{Z}+\mathbf{Z}^{\dagger}\right)^{-1} \overline{\mathbf{E E}^{\dagger}} \text {. }
$$

We therefore define:

$$
\text { Characteristic-noise matrix }=\mathbf{N} \equiv-\frac{1}{2}\left(\mathbf{Z}+\mathbf{Z}^{\dagger}\right)^{-1} \overline{\mathbf{E E}^{\dagger}}
$$

and conclude that:

The stationary values of the exchangeable power $P_{e, i}$ are the negatives of the (real) eigenvalues of the characteristic-noise matrix $\mathbf{N}$. 


\subsection{Properties of the Eigenvalues of the Characteristic-Noise Matrix in Impedance Form}

We shall now confirm the assertion made earlier (Sec. 2.3) to the effect that:

The exchangeable power of the n-terminal-pair network,

$$
P_{e}=\frac{1}{2} \overline{\left[\mathbf{E}^{\dagger}\left(\mathbf{Z}+\mathbf{Z}^{\dagger}\right)^{-1} \mathbf{E}\right]},
$$

is equal to the algebraic sum of the stationary values of $P_{e, i}$, which is alternatively the negative of the trace of the characteristic-noise matrix $\mathbf{N}$.

Setting $\mathbb{W}=\frac{1}{2}\left(\mathbf{Z}+\mathbf{Z}^{\dagger}\right)^{-1}$, we express the typical $i j$ th element of the matrix $\frac{1}{2}\left(\mathbf{Z}+\mathbf{Z}^{\dagger}\right)^{-1} \overline{\mathbf{E E}^{\dagger}}=\overline{\mathbf{W}} \overline{\mathbf{E} \mathbf{E}^{\dagger}}$ in the form

$$
\left(\mathbf{W} \overline{\mathbf{E E}^{\dagger}}\right)_{i j}=\sum_{k=1}^{n} W_{i k} \overline{E_{k} E_{j}^{*}}
$$

so that its trace (sum of diagonal elements) becomes

$$
\operatorname{Trace}\left(\mathbf{W} \overline{\mathbf{E E}}{ }^{\dagger}\right)=\sum_{k, l=1}^{n} W_{l k} \overline{E_{k} E_{l}{ }^{*}}=-\operatorname{trace}(\mathbf{N})
$$

But $P_{e}$ of the $n$-terminal-pair network equals

$$
P_{e}=\frac{1}{2} \overline{\left[\mathbf{E}^{\dagger}\left(\mathbf{Z}+\mathbf{Z}^{\dagger}\right)^{-1}\right] \mathbf{E}}=\overline{\mathbf{E}^{\dagger} \mathbf{W E}}=\sum_{k, l=1}^{n} \overline{E_{l}^{*} W_{l k} E_{k}}
$$

Comparing Eq. 3.17 with Eq. 3.16, we find

$$
P_{e}=-\operatorname{trace} \mathbf{N}=-\sum_{s} \lambda_{s}
$$

since the trace of a matrix is the sum of its eigenvalues.

We must now determine the ranges of values that can be assumed by the eigenvalues $\lambda_{s}$ of the characteristic-noise matrix $\mathbf{N}$ as well as the corresponding ranges of $P_{e, i}$. We first recall that the eigenvalues $\lambda_{s}$ determine the stationary values of $p$ in Eq. 3.10. The numerator of this expression is never negative, since $\mathbf{A}\left(=\overline{\mathbf{E} \mathbf{E}^{\dagger}}\right)$ is positive (semi) definite. Thus, the algebraic sign of $p$ is determined by that of the denominator. This in turn depends upon the definite character of $\mathbf{B}$, which is equal to $-2\left(\mathbf{Z}+\mathbf{Z}^{\dagger}\right)$. As noted previously, three cases have to be distinguished, in accordance with the second column of Table 3.1. In the first case, the denominator is always negative. Accordingly, the eigenvalues $\lambda_{s}$ pertaining to this case must all be negative, as shown in the last column of the table. The other cases follow in a similar manner. 
Table 3.1. Classification of Networks and Eigenvalues

\begin{tabular}{|c|c|c|c|c|}
\hline Case & $\mathbf{z}+\mathbf{z}^{\dagger}$ & Network Class & $p=-P_{e, i}$ & $\begin{array}{l}\text { Eigenvalues } \\
\left(\lambda_{s}\right) \text { of } \mathbf{N}\end{array}$ \\
\hline $\begin{array}{l}1 \\
2\end{array}$ & $\begin{array}{l}\text { positive definite } \\
\text { negative definite }\end{array}$ & $\begin{array}{l}\text { passive } \\
\text { active (negative resistance) }\end{array}$ & $\begin{array}{l}<0 \\
>0\end{array}$ & $\begin{array}{l}\text { all }<0 \\
\text { all }>0\end{array}$ \\
\hline 3 & indefinite & active & $\gtrless 0$ & $\left\{\begin{array}{l}\text { some }>0 \\
\text { some }<0\end{array}\right\}$ \\
\hline
\end{tabular}

The permissible values of $\lambda_{s}$ determine the ranges of values that $p$ can assume as a function of $\mathbf{x}$ in Eq. 3.10. Let us consider Case 1 first. No eigenvalue is positive. Among the eigenvalues, there is one of least magnitude (possibly zero) and another of largest magnitude. Since $p$ is a

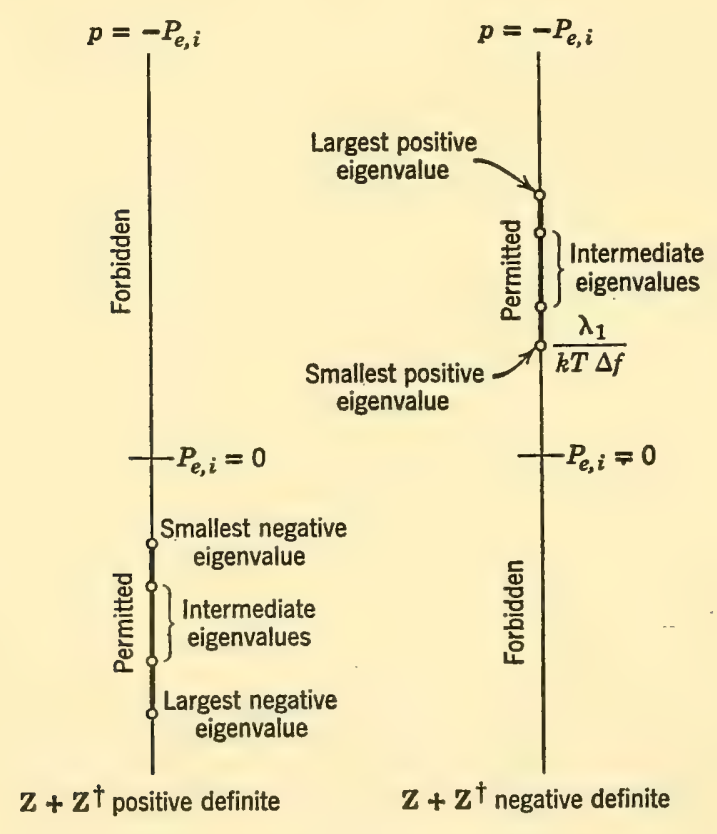

(a)

(b)

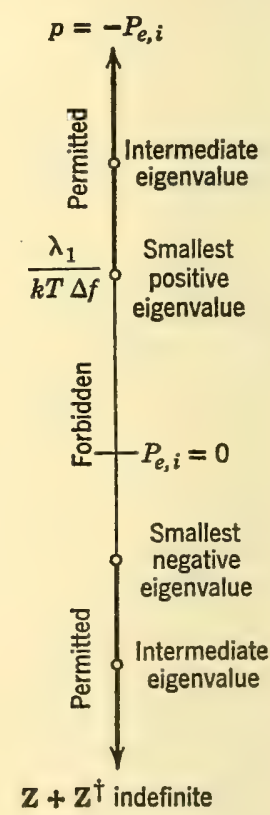

(c)

Fig. 3.1. Schematic diagram of permitted values of $p$ for four-terminal-pair networks.

continuous function of $\mathbf{x}$, its values lie between these two extreme eigenvalues, as illustrated in Fig. 3.1a. Analogous reasoning applies to the second case, illustrated in Fig. 3.1b.

Case 3 is a little more involved. The denominator of Eq. 3.10 can certainly become zero for some values of $\mathbf{x}$. Correspondingly, infinite values of $p$ will occur. Among the eigenvalues, there is a smallest 
positive one and a negative one of smallest magnitude. (In special cases, one or both may be equal to zero.) Again, because of the continuous nature of $p$ as a function of $\mathbf{x}, p$ may never take a value between and distinct from the foregoing extreme eigenvalues. The gap between the ranges of allowed values of $p$ is illustrated in Fig. 3.1c.

One particular property of the eigenvalues of $\mathbf{N}$ will be of importance later. Suppose that the original network with the characteristic-noise matrix $\mathbf{N}$ is imbedded in a $2 n$-terminal-pair lossless network, as shown in Fig. 2.2. A new $n$-terminal-pair network results, with the characteristicnoise matrix $\mathbf{N}^{\prime}$. The eigenvalues of $\mathbf{N}^{\prime}$ are the stationary values of the exchangeable power obtained in a subsequent imbedding of the type shown in Fig. 2.4. This second imbedding network is completely variable. One possible variation removes the first $2 n$-terminal-pair imbedding. Accordingly, the stationary values of the exchangeable power at the $(n+1)$ th terminal pair in Fig. 2.4 do not change when a $2 n$-terminal-pair lossless transformation is interposed between the two networks shown. It follows that:

The eigenvalues of the characteristic noise matrix $\mathbf{N}$ are invariant to a lossless transformation that preserves the number of terminal pairs.

At this point application of our results to two familiar examples of linear networks helps to establish further significance for the characteristic-noise matrix and its eigenvalues.

If the $n$-terminal-pair network contains only coherent (signal) generators rather than noise generators, $\overline{E_{i} E_{k}{ }^{*}}=E_{i} E_{k}{ }^{*}$ because ensemble averaging is unnecessary. The matrix $\overline{\mathbf{E E}^{\dagger}}$ is then of rank one; that is, a determinant formed of any submatrix of order greater than one is zero because its rows (or columns) are all proportional (Eq. 2.1). The rank of $\mathbf{N}$ cannot exceed that of either of its factors, so it too is of rank one (zero in a trivial case). Matrix $\mathbf{N}$ therefore has only one nonzero eigenvalue, and this is equal to trace $\mathbf{N}$. From Eq. 3.18, we conclude that for such networks, containing only coherent signal generators, the operations defined by Fig. 2.4 lead merely to the exchangeable power for the whole network (in the sense of Eq. 2.15). It is the single stationary value of $P_{e, i}$ and also the negative of the sole eigenvalue of $\mathbf{N}$.

Another simple but quite different case arises if the original (nonreciprocal) network is a passive one with dissipation, $\left(\mathbf{Z}+\mathbf{Z}^{\dagger}\right)$ positive definite, in thermal equilibrium at absolute temperature $T$. Then the operations defined by Fig. 2.4 must, on thermodynamic grounds, always lead to $P_{e, i}=k T \Delta f$ in a frequency band $\Delta f$, where $k$ is Boltzmann's constant. No matter what form the variable lossless network may take, 
$P_{e, i}$ must remain constant at the foregoing value. Thus, from Eq. 3.9,

$$
\mathbf{x}^{\dagger}\left[\overline{\mathbf{E E}^{\dagger}}-2 k T \Delta f\left(\mathbf{Z}+\mathbf{Z}^{\dagger}\right)\right] \mathbf{x} \equiv 0, \quad \text { for all } \mathbf{x}
$$

or

$$
\overline{\mathbf{E E}^{\dagger}}=2 k T \Delta f\left(\mathbf{Z}+\mathbf{Z}^{\dagger}\right)
$$

a result proved previously by Twiss. ${ }^{1}$ In terms of the characteristicnoise matrix $\mathbf{N}$, we have

$$
\mathbf{N}=-\frac{1}{2} \overline{\mathbf{E E}^{\dagger}}\left(\mathbf{Z}+\mathbf{Z}^{\dagger}\right)^{-1}=-k T \Delta f \mathbf{1}
$$

an equation indicating that a passive dissipative network at equilibrium temperature $T$ always has a diagonal $\mathbf{N}$ matrix, with all the eigenvalues equal to $-k T \Delta f$.

\subsection{Lossless Reduction in the Number of Terminal Pairs}

An $n$-terminal-pair network has a characteristic noise matrix of $n$th order, with $n$ eigenvalues. If $k$ of the $n$ terminal pairs of the network are left open-circuited and only the remaining $(n-k)$ terminal pairs are used, the original network is reduced to an $(n-k)$-terminal-pair network. This operation may be thought of as a special case of a more general reduction, achieved by imbedding the original $n$-terminal-pair network in a lossless $(2 n-k)$-terminal-pair network to produce $(n-k)$ available terminal pairs (see Fig. 3.2a). The case for $n-k=1$ was considered in Fig. 2.4.

The characteristic-noise matrix of the $(n-k)$-terminal-pair network has $(n-k)$ eigenvalues, which determine the extrema of the exchangeable power $P_{e, n-k+1}$ obtained in a subsequent (variable) reduction to one terminal pair (Fig. 3.2b). The successive reduction of the $n$-terminalpair network first to $(n-k)$ terminal pairs, and then to one terminal pair, is a special case of a direct reduction of the original network to one terminal pair. Comparison of the dotted box in Fig. $3.2 b$ with Fig. 2.4 shows that the exchangeable power $P_{e, n-k+1}$ obtained by the two successive reductions, with variation of only the second network (Fig. 3.2b), must lie within the range obtained by direct reduction with variation of the entire transformation network (Fig. 2.4). Hence the stationary values of $P_{e, n-k+1}$ found in Fig. $3.2 b$ must lie within the range prescribed for $P_{e, n+1}$ by Fig. 2.4. It follows that the eigenvalues of $\mathbf{N}$ for the $(n-k)$-terminal-pair network must lie within the allowed range of $P_{e, i}$ for the original $n$-terminal-pair network, illustrated in Fig. 3.1.

${ }^{1}$ R. Q. Twiss, "Nyquist's and Thévenin's Theorem Generalized for Nonreciprocal Linear Networks," J. Appl. Phys. 26, 599 (1955). 


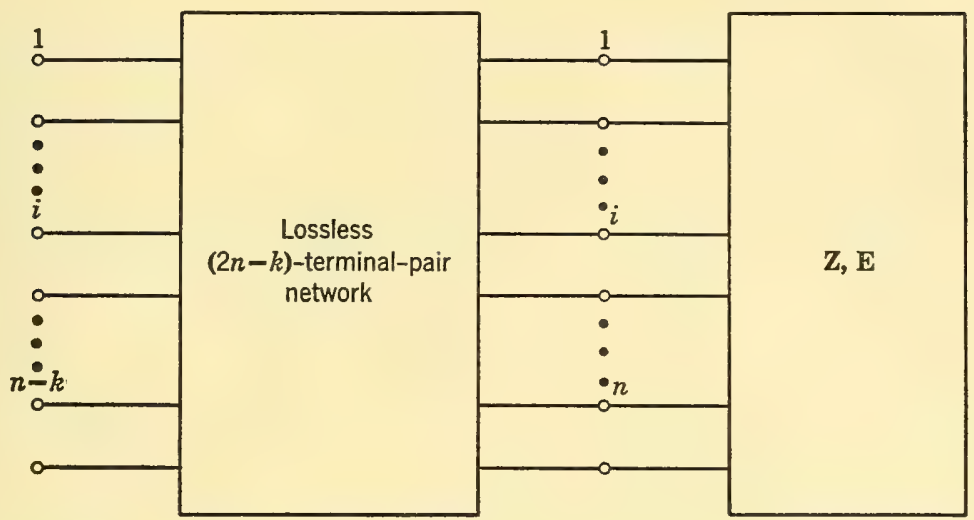

Fig. 3.2a. Reduction of $n$-terminal-pair network to $(n-k)$ terminal pairs.

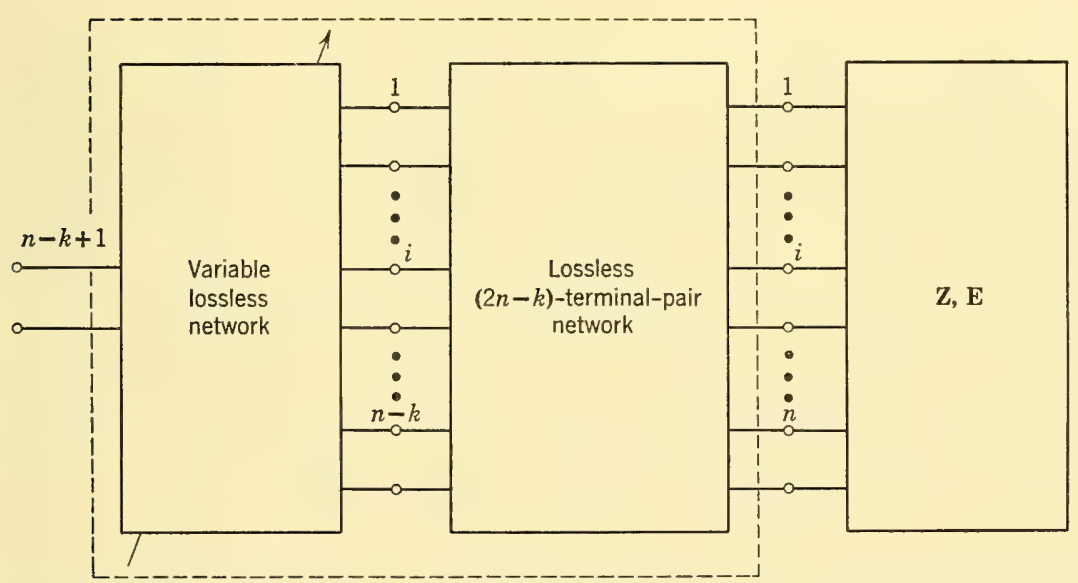

Fig. 3.2b. Successive reductions of $n$-terminal-pair network to $(n-k)$ terminal pairs and one terminal pair.

Since the number of terminal pairs is changed in the lossless reduction just considered, the theorem on the preservation of the eigenvalues under lossless transformations (p. 25) does not apply. While the new eigenvalues of the reduced network do lie within the range of the original ones, their values will usually be different. 


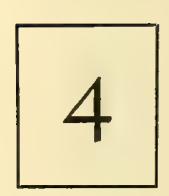

\section{Canonical Form}

\section{of Linear Noisy Networks}

Lossless network transformations performed on a noisy network, in such a way that the number of terminal pairs is unchanged, change the impedance matrix as well as the noise spectra. However, these lossless network transformations do not change the eigenvalues of the characteristic noise matrix. Thus we know that each noisy network possesses some essential noise characteristics, unalterable by those lossless network transformations which preserve the number of terminal pairs. On this basis, we expect to be able to find a fundamental form of the network which places these characteristics directly in evidence. In this chapter, we shall develop such a form of the network. This fundamental or "canonical" network form is, of course, attainable through lossless network transformations performed on the original network. The existence of a canonical form for every linear noisy network greatly clarifies its most important noise characteristics and simplifies the study of fundamental limits on its noise performance. Since the canonical network contains not more than $n$ real parameters for every $n$-terminal-pair network, its existence also shows that an $n$-terminal-pair, linear noisy network does not possess more than $n$ (real) invariants with respect to lossless transformations.

\subsection{Derivation of the Canonical Form}

In this section we shall prove the following theorem:

At any particular frequency, every n-terminal-pair network can be reduced by lossless imbedding into a canonical form consisting of $n$ separate 
(possibly negative) resistances in series with uncorrelated noise voltage generators.

We note first that a lossless imbedding of the $n$-terminal-pair network transforms the two matrices $\mathbf{Z}+\mathbf{Z}^{\dagger}$ and $\overline{\mathbf{E E}^{\dagger}}$ in identical colinear manner, as shown in Eqs. 3.3 and 3.6. We have also noted that the matrix $\tau$ which appears in the transformation is entirely unrestricted by the conditions (Eqs. 2.5) of losslessness for the imbedding network.

It is always possible to diagonalize simultaneously two Hermitian matrices, one of which $\left(\overline{\mathbf{E E}}{ }^{\dagger}\right)$ is positive definite (or, as a limiting case, semidefinite), by one and the same colinear transformation. Thus, suppose that both $\mathbf{Z}^{\prime}+\mathbf{Z}^{\prime \dagger}$ and $\overline{\mathbf{E}^{\prime} \mathbf{E}^{\prime \dagger}}$ have been diagonalized by a proper imbedding of the original network (see Fig. 2.2). This means that the impedance matrix $\mathbf{Z}^{\prime}$ of the resulting network is of the form

$$
\mathbf{Z}^{\prime}=\operatorname{diag}\left(R_{1}, R_{2}, \cdots, R_{n}\right)+\mathbf{Z}_{\text {rem }}
$$

where $\mathbf{Z}_{\mathrm{rem}}$ fulfills the condition of the impedance matrix of a lossless network $\mathbf{Z}_{\mathrm{rem}}=-\left(\mathbf{Z}_{\mathrm{rem}}\right)^{\dagger}$.

Suppose, finally, that a lossless (and therefore noise-free) network with the impedance matrix $-\mathbf{Z}_{\mathrm{rem}}$ is connected in series with our network $\left(\mathbf{Z}^{\prime}, \mathbf{E}^{\prime}\right)$, as shown in Fig. 4.1. The result is a network with the impedance matrix

$$
\mathbf{Z}^{\prime \prime}=\mathbf{Z}^{\prime}-\mathbf{Z}_{\text {rem }}=\operatorname{diag}\left(R_{1}, R_{2}, \cdots, R_{n}\right)
$$

The open-circuit noise voltages remain unaffected when a lossless sourcefree network is connected in series with the original network. Thus,

$$
\mathbf{E}^{\prime \prime}=\mathbf{E}^{\prime} \quad \text { or } \quad \overline{\mathbf{E}^{\prime \prime} \mathbf{E}^{\prime \prime \dagger}}=\overline{\mathbf{E}^{\prime} \mathbf{E}^{\prime \dagger}}
$$

Consequently, the two operations lead to a network with the diagonal impedance matrix $\mathbf{Z}^{\prime \prime}$ of Eq. 4.2 and a diagonal noise matrix $\overline{\mathbf{E}^{\prime \prime} \mathbf{E}^{\prime \prime t}}$. This canonical form of the network consists of $n$ separate resistances in series with uncorrelated noise voltage generators, as shown in Fig. 4.2.

Noting that the series connection of a lossless network is a special case of a lossless imbedding, we have proved the theorem stated at the beginning of this section.

A lossless imbedding leaves the eigenvalues of the characteristic-noise matrix invariant. Thus, the eigenvalues of the characteristic-noise matrix $\mathbf{N}^{\prime \prime}$ of the canonical form of the original network are equal to those of $\mathbf{N}$ of the original network. But, the eigenvalues $\lambda_{n}$ of $\mathbf{N}^{\prime \prime}$ are clearly its $n$ diagonal elements

$$
\lambda_{k}=-\frac{\overline{E_{k}{ }^{\prime \prime} E_{k}{ }^{\prime \prime \prime}}}{4 R_{k}}
$$


The $k$ th eigenvalue is the negative of the exchangeable power of the $k$ th source. Thus, we have proved the following theorem:

The exchangeable powers of the $n$ Thervenin sources of the canonical form of any n-terminal-pair network are equal and opposite in sign to the $n$ eigenvalues of the characteristic noise matrix $\mathbf{N}$ of the original network.

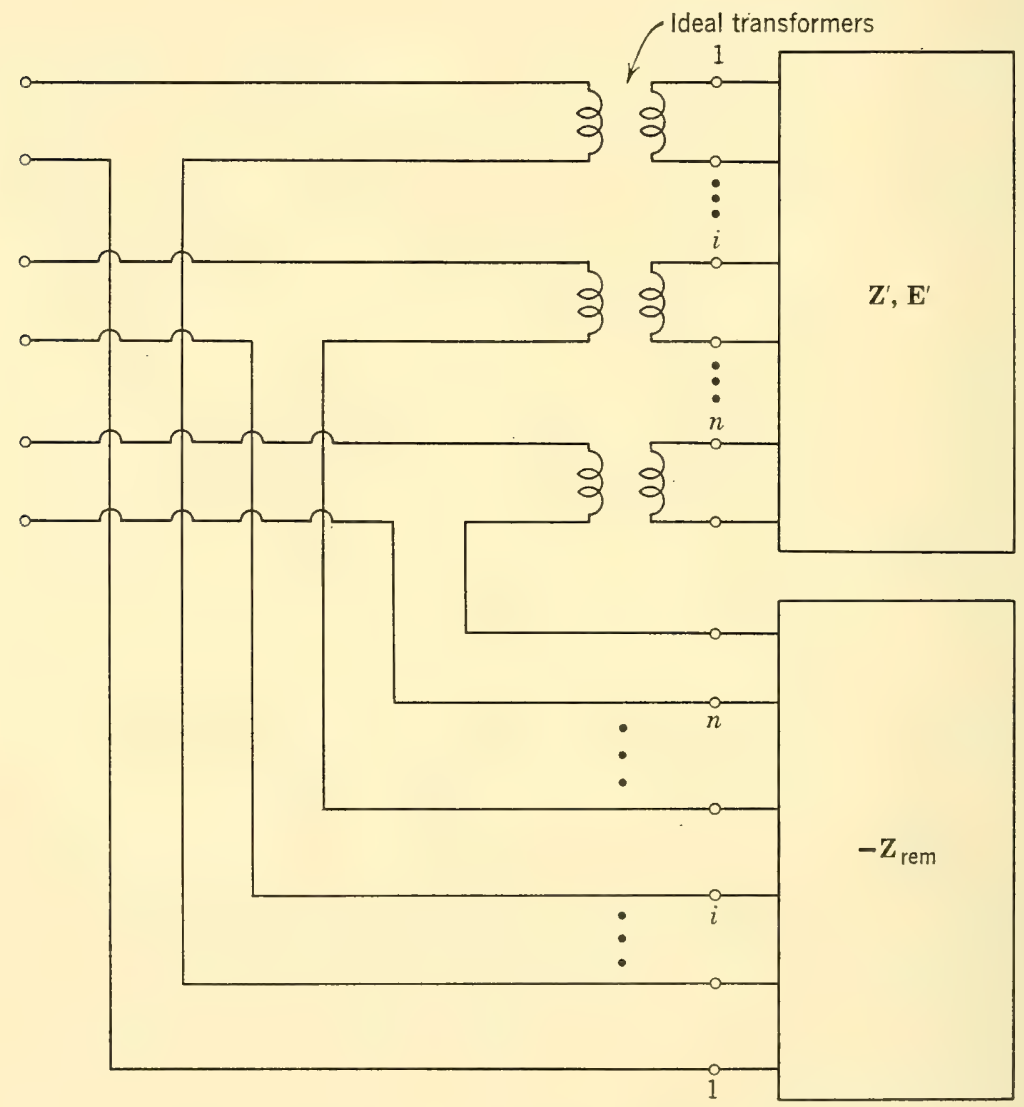

Fig. 4.1. Series connection of networks $\left(Z^{\prime}, E^{\prime}\right)$ and $-Z_{\text {rem. }}$.

Since ideal transformers may be applied to each terminal pair of the foregoing canonical form in a manner that reduces either all resistors or all $\left|E_{k}\right|$ to unit magnitude, there are actually only $n$ independent (real) parameters contained in the canonical form. This fact proves the following theorem: 
A linear noisy n-terminal-pair network possesses not more than $n$ invariants with respect to lossless transformations, and these are all real numbers.

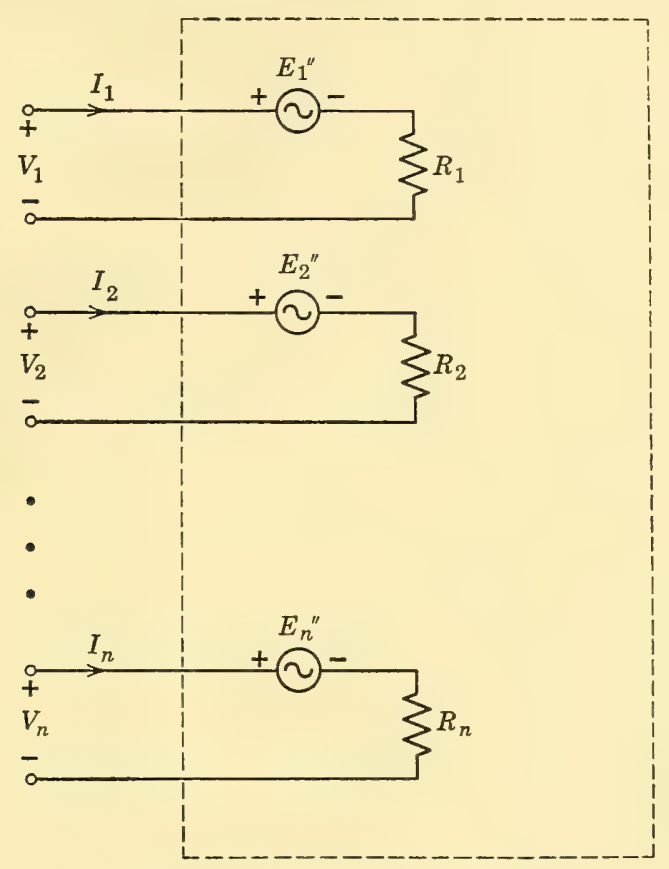

Fig. 4.2. The canonical network.

\subsection{Interconnection of Linear Noisy Networks}

The canonical form is helpful in simplifying the discussion of the interconnection of noisy networks. Consider an $n$-terminal-pair noisy network and an independently noisy $m$-terminal-pair network. Let them be connected through a $2(m+n)$-terminal-pair lossless network, resulting in an $(m+n)$-terminal-pair network, as shown in Fig. 4.3. We shall now determine the eigenvalues of the characteristic-noise matrix $\mathbf{N}_{m+n}$ of the resulting $(m+n)$-terminal-pair network.

To do so, we first reduce each of the component networks to canonical form of the type shown in Fig. 4.2. This procedure places in evidence, but does not alter, the eigenvalues of their respective characteristic-noise matrices. Taken together, the two canonical forms represent the canonical form of the $(m+n)$-terminal-pair network of Fig. 4.3. Accordingly, the $m+n$ eigenvalues of that network are merely the eigenvalues of the component networks. The proof obviously covers the interconnection 
of any number of independently noisy networks of any size, provided the total number of terminal pairs is preserved.

We shall be interested in cases in which the number of terminal pairs is reduced upon interconnection. A simple example is the interconnection

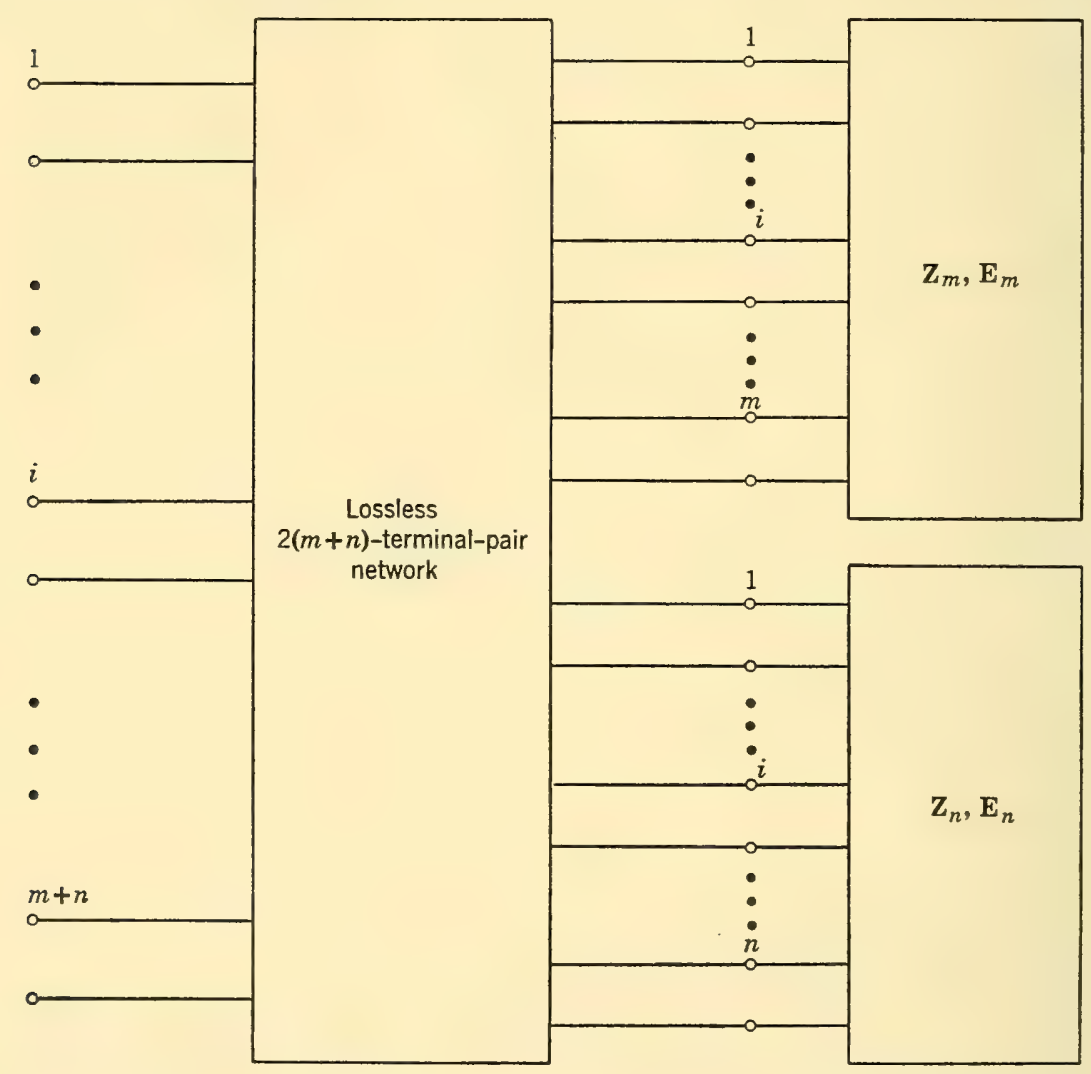

Fig. 4.3. Lossless interconnection of $m$-terminal-pair and $n$-terminal-pair networks.

of several two-terminal-pair amplifiers, with feedback, to form a new two-terminal-pair amplifier. In such a case, the effect of the reduction can be understood by applying the reasoning of Sec. 3.4 to the available terminals of the network in Fig. 4.3. The conclusion is immediate.

The eigenvalues of the characteristic-noise matrix of an n-terminal-pair network constructed by lossless interconnection of independently noisy component networks having $m$ terminal pairs in all, $m>n$, must lie within the range defined by the most extreme eigenvalues of the component networks. 


\section{5}

\section{Linear Noisy Networks in Other Representations}

In the foregoing analysis we have found all the invariants of a linear noisy network with respect to lossless imbeddings that preserve the number of terminal pairs. With the aid of the impedance formalism, these invariants have been interpreted in terms of the exchangeable power, on the one hand, and in terms of the canonical representation of the network, on the other. There are, however, additional interpretations of the invariants, which are brought out by different matrix representations of the network. For each new representation a characteristic-noise matrix can be defined. As we might expect, all such characteristic-noise matrices have the same eigenvalues, since, after all, these are the only invariants of the network.

\subsection{General Matrix Representations}

The impedance-matrix representation, Eq. 2.6, is conveniently rewritten in the form ${ }^{1}$

$$
\left[\begin{array}{l:l}
1 & -\mathrm{Z}
\end{array}\right]\left[\begin{array}{c}
\mathrm{V} \\
-- \\
\mathrm{I}
\end{array}\right]=\mathrm{E}
$$

where $\mathbf{I}$ is the identity matrix of the same order as $\mathbf{Z}$. Any other matrix

${ }^{1}$ V. Belevitch, "Four-Dimensional Transformations of 4-pole Matrices with Applications to the Synthesis of Reactance 4-poles," IRE Trans. on Circuit Theory, CT-3, No. 2, 105 (1956). 
representation of a linear noisy network can be expressed as

$$
\mathbf{v}-\mathbf{T u}=\delta
$$

where $\mathbf{v}$ is a column matrix consisting of the amplitudes of the terminal "response," $\mathbf{u}$ is the corresponding column matrix of the terminal "excitation," and $\delta$ is a column matrix comprising the amplitudes of the internal (noise) sources as seen at the terminals. The square matrix $\mathbf{T}$ expresses the transformation of the network in the absence of internal sources.

As an example of such a matrix representation of a $2 m$-terminal-pair network, we consider the mixed voltage-current representation, for which

$$
\begin{gathered}
\mathbf{v}=\left[\begin{array}{c}
V_{1} \\
I_{1} \\
V_{3} \\
I_{3} \\
\cdot \\
\cdot \\
\cdot \\
V_{2 m-1} \\
I_{2 m-1}
\end{array}\right] \\
\mathbf{u}=\left[\begin{array}{c}
V_{2} \\
-I_{2} \\
V_{4} \\
-I_{4} \\
\cdot \\
\cdot \\
\cdot \\
V_{2 m} \\
-I_{2 m}
\end{array}\right]
\end{gathered}
$$

and $\delta$ is the noise column vector

$$
\delta=\left[\begin{array}{c}
E_{n 1} \\
I_{n 1} \\
E_{n 3} \\
I_{n 3} \\
\cdot \\
\cdot \\
\cdot \\
E_{n(2 m-1)} \\
I_{n(2 m-1)}
\end{array}\right]
$$

The equivalent circuit suggested by the representation, Eq. 5.2, with the interpretations, Eqs. 5.3, 5.4, and 5.5, is shown in Fig. 5.1. 


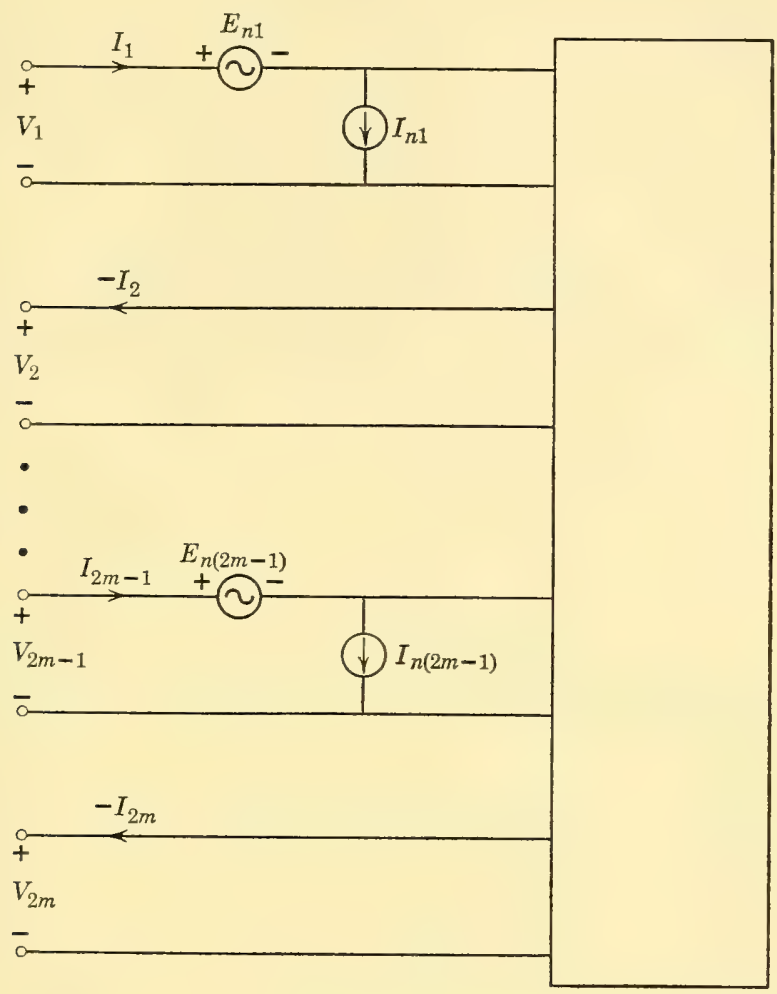

Fig. 5.1. Mixed voltage-current representation of $2 m$-terminal-pair network.

Returning to the general expression (Eq. 5.2), we note that it also can be written in a form similar to Eq. 5.1.

$$
\left[\begin{array}{l:c}
1 & -\mathbf{T}
\end{array}\right]\left[\begin{array}{c}
\mathbf{v} \\
-- \\
\mathbf{u}
\end{array}\right]=\delta
$$

The analysis in Sec. 5.2 shows how transformations are performed from one matrix representation to another. Then the transformation of the power expression will be studied. Combining the results of these two studies, we shall be able to define a characteristic-noise matrix for every formalism and to show its relation to the characteristic-noise matrix defined in Eq. 3.15.

\subsection{Transformation from One Matrix Representation to Another} The variables $\left[\begin{array}{c}\mathbf{V} \\ -- \\ \mathbf{I}\end{array}\right]$ and $\left[\begin{array}{c}\mathbf{v} \\ -- \\ \mathbf{u}\end{array}\right]$ can always be related by a linear trans- 
formation of the form

$$
\mathbf{R}\left[\begin{array}{c}
\mathbf{v} \\
-- \\
\mathbf{u}
\end{array}\right]=\left[\begin{array}{c}
\mathbf{V} \\
-- \\
\mathbf{I}
\end{array}\right]
$$

where $\mathbf{R}$ is a square matrix of order twice that of either $\mathbf{V}$ or $\mathbf{I}$. For a $2 m$-terminal-pair network, $\mathbf{R}$ is of order $4 m$. For example, the transformation from the impedance representation of a 2-terminal-pair network into the general-circuit-parameter representation (Eqs. 5.3, 5.4, and 5.5) yields

$$
\mathbf{R}=\left[\begin{array}{rrrr}
1 & 0 & 0 & 0 \\
0 & 0 & 1 & 0 \\
0 & 1 & 0 & 0 \\
0 & 0 & 0 & -1
\end{array}\right]
$$

The relation between the matrices $\mathbf{Z}$ and $\mathbf{T}$ is derived in the following way. We start from Eq. 5.1 and introduce the transformation (Eq. 5.7):

$$
\left[\begin{array}{l:l}
\mathbf{1} & -\mathbf{Z}
\end{array}\right] \mathbf{R R}^{-1}\left[\begin{array}{c}
\mathbf{V} \\
-- \\
\mathbf{I}
\end{array}\right]=\mathbf{E}
$$

or

$$
\left[\begin{array}{l:l}
\mathbf{1} & -\mathbf{Z}
\end{array}\right] \mathbf{R}\left[\begin{array}{c}
\mathbf{v} \\
-- \\
\mathbf{u}
\end{array}\right]=\mathbf{E}
$$

In order to relate Eq. 5.9 to Eq. 5.2, we note that the order of $\mathbf{R}$ is twice that of $\mathbf{Z}$. The matrix $\mathbf{R}$ is therefore conveniently split into submatrices as

$$
\mathbf{R}=\left[\begin{array}{c:c}
\mathbf{R}_{11} & \mathbf{R}_{12} \\
\hdashline \mathbf{R}_{21} & \mathbf{R}_{22}
\end{array}\right]
$$

where the $\mathbf{R}_{i j}$ are of the same order as $\mathbf{Z}$. Carrying out the multiplication in Eq. 5.9, we obtain

$$
\left[\begin{array}{l:l}
\mathbf{R}_{11}-\mathbf{Z} \mathbf{R}_{21} & \mathbf{R}_{12}-\mathbf{Z} \mathbf{R}_{22}
\end{array}\right]\left[\begin{array}{c}
\mathbf{v} \\
-- \\
\mathbf{u}
\end{array}\right]=\mathbf{E}
$$

The correspondence between Eqs. 5.11 and 5.2 is made complete if we multiply Eq. 5.11 by

$$
\mathbf{M}=\left[\mathbf{R}_{11}-\mathbf{Z R}_{21}\right]^{-1}
$$


obtaining

$$
\left[\begin{array}{c:c}
\mathbf{1} & -\mathbf{T}
\end{array}\right]\left[\begin{array}{c}
\mathbf{v} \\
-- \\
\mathbf{u}
\end{array}\right]=\delta
$$

where

$$
\left[\begin{array}{l:l}
1 & -\mathbf{T}
\end{array}\right]=\mathbf{M}\left[\begin{array}{l:l}
1 & -Z
\end{array}\right] \mathbf{R}
$$

and

$$
\delta=\mathbf{M E}
$$

Equations 5.12 to 5.14 summarize the transformation from one matrix representation to another.

\subsection{Power Expression and Its Transformation}

In any matrix representation, the power $P$ flowing into the network is a real quadratic form of the excitation-response vector $\left[\begin{array}{c}\mathbf{v} \\ -- \\ \mathbf{u}\end{array}\right]$. We have

$$
P=\left[\begin{array}{c}
\mathbf{v} \\
-- \\
\mathbf{u}
\end{array}\right]^{\dagger} \mathbf{Q}_{T}\left[\begin{array}{c}
\mathbf{v} \\
-- \\
\mathbf{u}
\end{array}\right]
$$

where $\mathbf{Q}_{T}$ is a Hermitian matrix of order twice that of either $\mathbf{u}$ or $\mathbf{v}$. In the particular case of the impedance-matrix representation,

$$
\begin{aligned}
P & =\frac{1}{2}\left[\mathbf{V}^{\dagger} \mathbf{I}+\mathbf{I}^{\dagger} \mathbf{V}\right] \\
& =\frac{1}{2}\left[\begin{array}{c}
\mathbf{V} \\
\hdashline \\
\mathbf{I}
\end{array}\right]^{\dagger}\left(\begin{array}{c:c}
\mathbf{0} & \mathbf{1} \\
-\mathbf{1} & \mathbf{0}
\end{array}\right)\left[\begin{array}{c}
\mathbf{V} \\
-- \\
\mathbf{I}
\end{array}\right]
\end{aligned}
$$

Comparing Eqs. 5.16 and 5.15, we find that the $\mathbf{Q}$ matrix for the impedance representation is

$$
\mathbf{Q}_{z}=\frac{1}{2}\left[\begin{array}{c:c}
0 & 1 \\
-1 & 0
\end{array}\right]
$$

A transformation from one representation into another transforms the $\mathbf{Q}$ matrix. Let us study how $\mathbf{Q}$ changes when we transform from the impedance representation into the general representation of Eq. 5.6. 
We have

$$
\begin{aligned}
P & =\left[\begin{array}{c}
\mathbf{V} \\
-- \\
\mathbf{I}
\end{array}\right]^{\dagger} \mathbf{Q}_{z}\left[\begin{array}{c}
\mathbf{V} \\
-- \\
\mathbf{I}
\end{array}\right] \\
& =\left[\begin{array}{c}
\mathbf{v} \\
-- \\
\mathbf{u}
\end{array}\right]^{\dagger} \mathbf{R}^{\dagger} \mathbf{Q}_{z} \mathbf{R}\left[\begin{array}{c}
\mathbf{v} \\
-- \\
\mathbf{u}
\end{array}\right]
\end{aligned}
$$

Comparison with Eq. 5.15 shows that

$$
\mathbf{Q}_{T}=\mathbf{R}^{\dagger} \mathbf{Q}_{Z} \mathbf{R}
$$

where $\mathbf{R}$ is the matrix that transforms the general-excitation vector $\left[\begin{array}{c}\mathbf{v} \\ -- \\ \mathbf{u}\end{array}\right]$ into the voltage and current vector $\left[\begin{array}{c}\mathbf{V} \\ -- \\ \mathbf{I}\end{array}\right]$, according to Eq. 5.7.

We are now ready to set up the general characteristic-noise matrix $\mathbf{N}$ for any matrix representation, Eq. 5.6.

\subsection{The General Characteristic-Noise Matrix}

We have introduced the most general matrix representation of a linear network in Eq. 5.6. We have defined the associated power matrix $\mathbf{Q}_{T}$ in Eq. 5.15. With these two we may define a general characteristic-noise matrix $\mathbf{N}_{T}$ corresponding to this matrix representation. The requirements are that this matrix $\mathbf{N}_{T}$ :

1. Should reduce exactly to the form of Eq. 3.15 when the network is described on the impedance basis.

2. Should be related to Eq. 3.15 by a similarity transformation when the same network is described on other than impedance basis (for example, admittance, scattering, and so forth).

Under these conditions, $\mathbf{N}_{T}$ will contain the $n$ network invariants as its eigenvalues.

Here we shall follow the simple expedient of defining $\mathbf{N}_{T}$ and then proving its relationship to the matrix defined by Eq. 3.15. Thus, let

$$
\mathbf{N}_{T}=\left\{[\mathbf{1}:-\mathbf{T}] \mathbf{Q}_{T}^{-1}\left[\begin{array}{l:l}
\mathbf{1} & -\mathbf{T}
\end{array}\right]^{\dagger}\right\}^{-1} \overline{\delta \delta^{\dagger}}
$$

For the impedance-matrix representation, using Eqs. 5.1, 5.6, and 5.17 , we obtain

$$
\left[\begin{array}{l:l}
\mathbf{1} & -\mathbf{Z}
\end{array}\right] \mathbf{Q}_{z^{-1}}^{-1}\left[\begin{array}{l:l}
\mathbf{1} & -\mathbf{Z}
\end{array}\right]^{\dagger}=-2\left(\mathbf{Z}+\mathbf{Z}^{\dagger}\right)
$$

Introducing Eq. 5.20 into Eq. 5.19, we have

$$
\mathbf{N}_{Z}=-\frac{1}{2}\left(\mathbf{Z}+\mathbf{Z}^{\dagger}\right)^{-1} \overline{\mathbf{E E}^{\dagger}}
$$


But, Eq. 5.21 is identical with the definition Eq. 3.15 for $\mathbf{N}$.

Next, let us relate the general noise matrix $\mathbf{N}_{T}$ of Eq. 5.19 to its particular form in the impedance representation. For this purpose, we note that according to Eq. 5.14

$$
\overline{\delta \delta^{\dagger}}=\overline{\mathbf{M E E ^ { \dagger }}} \mathbf{M}^{\dagger}
$$

Then, using Eqs. 5.13, 5.18, and 5.20, we find

$$
\begin{aligned}
& {\left[\begin{array}{l:l}
\mathbf{1} & \mathbf{- T}
\end{array}\right] \mathbf{Q}_{T}^{-1}[\mathbf{1}:-\mathbf{T}]^{\dagger}} \\
& =\mathbf{M}\left[\begin{array}{l:l}
\mathbf{1} & -\mathbf{Z}
\end{array}\right] \mathbf{R} \mathbf{Q}_{T}^{-1} \mathbf{R}^{\dagger}\left[\begin{array}{l:l}
\mathbf{1} & -\mathbf{Z}
\end{array}\right]^{\dagger} \mathbf{M}^{\dagger} \\
& =-2 \mathbf{M}\left(\mathbf{Z}+\mathbf{Z}^{\dagger}\right) \mathbf{M}^{\dagger}
\end{aligned}
$$

Combining Eqs. 5.21, 5.22, and 5.23 with 5.19, we have finally

$$
\mathbf{N}_{T}=\mathbf{M}^{\dagger-1} \mathbf{N}_{Z} \mathbf{M}^{\dagger}
$$

According to Eq. 5.24, the characteristic-noise matrix $\mathbf{N}_{T}$ of the general matrix representation of a network is related by a similarity transformation to the characteristic-noise matrix $\mathbf{N}_{Z}$ of the impedance-matrix representation of the same network. Therefore, $\mathbf{N}_{T}$ and $\mathbf{N}_{Z}$ have the same eigenvalues.

The eigenvalues of the characteristic-noise matrix of Eq. 5.21 determined the stationary values of the real quantity $p$ in Eq. 3.10. Comparison of these two expressions with the expression for the general characteristic-noise matrix in Eq. 5.19 shows that its eigenvalues determine the stationary values of the associated real quantity $p_{T}$

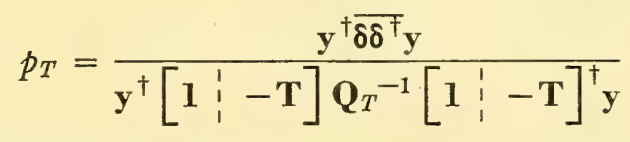

with respect to variations of the arbitrary column matrix $\mathbf{y}$. It is easily shown by the method of Sec. 3.2 that this "noise parameter" $p_{T}$ has, in fact, as extrema the eigenvalues of the matrix $\mathbf{N}_{T}$ defined in Eq. 5.19. The range of values that $p_{T}$ assumes as a function of $\mathbf{y}$ is identical with the range of values of $p$ in Eq. 3.10 and Fig. 3.1.

The network classification in the three cases illustrated in Fig. 3.1 should now be restated in terms of the T-matrix representation. This is easily accomplished by considering Eq. 5.23. According to it, the matrices $\left[\begin{array}{l:l}\mathbf{1} & \mathbf{- T}\end{array}\right] \mathbf{Q}_{T}^{-\mathbf{1}}\left[\begin{array}{l:l}\mathbf{1} & \mathbf{-} \mathbf{T}\end{array}\right]^{\dagger}$ and $-\left(\mathbf{Z}+\mathbf{Z}^{\dagger}\right)$ are related by a colinear transformation. A colinear transformation preserves the signature of a matrix. Consequently, the network classification of Table 3.1 can be carried out in the T-matrix representation, as shown in Table 5.1. The same conclusion may be reached from the facts that $\mathbf{N}_{T}$ and $\mathbf{N}_{Z}$ have the same eigenvalues, and both $\overline{\mathbf{E E}}$ and $\overline{\delta \delta^{\dagger}}$ are positive definite. 
Table 5.1. Classification of Networks and Eigenvalues in T-Matrix Representation

\begin{tabular}{|c|c|c|c|c|}
\hline $\begin{array}{c}\text { Case } \\
1\end{array}$ & 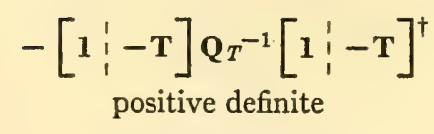 & $\begin{array}{l}\text { Network } \\
\text { Class } \\
\text { passive }\end{array}$ & $\begin{array}{l}p_{T} \\
<0\end{array}$ & $\begin{array}{l}\text { Eigenvalues } \\
\left(\lambda_{s}\right) \text { of } \mathbf{N}_{T} \\
\text { all }<0\end{array}$ \\
\hline 2 & negative definite & $\begin{array}{l}\text { active (negative } \\
\text { resistance) }\end{array}$ & $>0$ & all $>0$ \\
\hline 3 & indefinite & active & $\gtrless 0$ & $\left\{\begin{array}{l}\text { some } \geq 0 \\
\text { some } \leq 0\end{array}\right\}$ \\
\hline
\end{tabular}

Figure 3.1 gives directly the allowed range of $p_{T}$ and the eigenvalues of $\mathbf{N}_{T}$, if the notation of Table 3.1 is replaced on the figure by that of Table 5.1 .

In the specific case of the mixed voltage-current representation of Eqs. 5.3 to $5.5, \mathrm{~N}_{T}$ and $p_{T}$ can be simplified if we introduce the detailed expressions for the power matrix $\mathbf{Q}_{T}$. This matrix is found most directly from the explicit expression for the power $P$ flowing into the network in terms of the excitation-response vector $\left[\begin{array}{c}\mathbf{v} \\ -- \\ \mathbf{u}\end{array}\right]$. Comparing the resulting expression with Eq. 5.15 allows identification of $\mathbf{Q}_{T}$ by inspection. The power matrix $\mathbf{Q}_{T}$ is square and of $4 m$ th order

$$
\mathbf{Q}_{T}=\frac{1}{2}\left[\begin{array}{c:c}
\mathbf{P} & 0 \\
-0 & -\mathbf{P}
\end{array}\right]
$$

where the P's are matrices of order $2 m$ of the form

$$
\mathbf{P}=\left[\begin{array}{cc:cc:cc:c:cc}
0 & 1 & 0 & 0 & 0 & 0 & \cdots & 0 & 0 \\
1 & 0 & 0 & 0 & 0 & 0 & \cdots & 0 & 0 \\
\hdashline 0 & 0 & 0 & 1 & 0 & 0 & \cdots & 0 & 0 \\
0 & 0 & 1 & 0 & 0 & 0 & \cdots & 0 & 0 \\
\hdashline 0 & 0 & 0 & 0 & 0 & 1 & \cdots & 0 & 0 \\
0 & 0 & 0 & 0 & 1 & 0 & \cdots & 0 & 0 \\
\hdashline \cdot & . & . & . & . & . & \cdots & . & \cdot \\
. & . & . & . & . & . & \cdots & . & . \\
. & . & . & . & . & . & \cdots & . & . \\
-0 & 0 & 0 & 0 & 0 & 0 & \cdots & 0 & 1 \\
0 & 0 & 0 & 0 & 0 & 0 & \cdots & 1 & 0
\end{array}\right]
$$


It is easily checked that $\mathbf{P}$ has the properties

$$
\mathbf{P}^{-1}=\mathbf{P}^{\dagger}=\mathbf{P}
$$

Substituting the particular form of $\mathbf{Q}_{T}$ from Eq. $5.26 a$ into the matrix in column 2 of Table 5.1, we obtain the matrix of $2 m$ th order

$$
\left[\begin{array}{l:l}
\mathbf{1} & -\mathbf{T}
\end{array}\right] \mathbf{Q}_{T}^{-1}[\mathbf{1}:-\mathbf{T}]^{\dagger}=2\left(\mathbf{P}-\mathbf{T P T}^{\dagger}\right)
$$

Thus, from Eq. 5.19 we have for $\mathbf{N}_{T}$

$$
\mathbf{N}_{T}=\frac{1}{2}\left(\mathbf{P}-\mathbf{T} \mathbf{P} \mathbf{T}^{\dagger}\right)^{-1} \overline{\delta \delta^{\dagger}}
$$

With the introduction of the specific expression Eq. 5.28 into Eq. 5.25 for the noise parameter $p_{T}$, we find that it reduces to

$$
p_{T}=\frac{\mathbf{y}^{\dagger} \overline{\delta \delta^{\dagger} \mathbf{y}}}{2 \mathbf{y}^{\dagger}\left(\mathbf{P}-\mathbf{T P T}^{\dagger}\right) \mathbf{y}}
$$

The preceding development shows how each matrix representation $\mathbf{T}$ has associated with it a particular noise parameter $p_{T}$, of which the extrema are determined by the eigenvalues of its characteristic-noise matrix. In the next chapter we shall develop in detail the significance of $p_{T}$ for two-terminal-pair amplifiers represented in terms of their general circuit constants. 


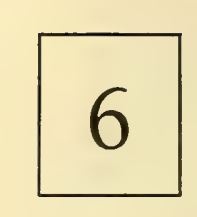

\section{Noise Measure}

In Chap. 3, starting from the impedance-matrix representation, we defined a noise matrix $\mathbf{N}_{Z}$. The eigenvalues of $\mathbf{N}_{Z}$ gave the extrema of a scalar, $p_{Z}$, which was found to be the exchangeable power derived from the polyterminal network under consideration by the arrangement of Fig. 2.4. In Chap. 5, we defined a generalized noise matrix $\mathbf{N}_{T}$, pertaining to the general matrix representation in Eq. 5.2. The eigenvalues of $\mathbf{N}_{T}$ and $\mathbf{N}_{Z}$ are identical. Associated with the eigenvalues of $\mathbf{N}_{T}$ are the extrema of the generalized scalar parameter $p_{T}$ in Eq. 5.25. Special forms of $\mathbf{N}_{T}$ and $p_{T}$ for the mixed voltage-current representation (Eqs. 5.3 through 5.6) were given in Eqs. 5.29 and 5.30. In the case of a twoterminal-pair network, this representation reduces to the "general-circuitconstant" description. Our interest in the noise performance of linear amplifiers gives the two-terminal-pair case a special importance. The remaining part of our work will therefore be confined to the interpretation and study of $p_{T}$ for the two-terminal-pair network in the generalcircuit-constant representation.

Our problem is to find the physical operation that leads to the extrema of $p_{T}$, in the same manner as the operation of Fig. 2.4 led to the extrema of $p_{Z}$. It is obvious that the operations involved in the extremization will make use of lossless imbeddings, since only such operations leave the eigenvalues of $\mathbf{N}_{T}$ unchanged. Variations in these imbeddings will presumably produce variations in the column vector $\mathbf{y}$ in Eq. 5.30, and lead to the extrema of $p_{T}$.

The general-circuit-constant representation of a two-terminal-pair network emphasizes its transfer characteristics. Therefore, we expect 
that $p_{T}$ in this representation must be related to the noise performance of the network regarded as a transfer device. The noise figure has been for many years the most widely used parameter describing the noise performance of transfer devices. Consequently, we might well investigate first whether or not $p_{T}$ has any relation with the noise figure.

\subsection{Extended Definitions of Gain and Noise Figure}

The noise figure is normally defined in terms of available power. We have seen, however, that the available-power concept leads to diffculties in cases involving negative resistances. Since such cases must arise in any general theory of linear amplifiers, the available-power concept should be replaced everywhere by the exchangeable power. Accordingly, the same replacement should be made in the available-gain definition: The exchangeable power $P_{e S}$ of the input source and the exchangeable power $P_{e O}$ at the network output replace the corresponding available powers. Specifically,

$$
G_{e}=\frac{P_{e O}}{P_{e S}}
$$

where

$$
P_{e O}=\frac{E_{O}^{2}}{4 R_{O}}
$$

and

$$
P_{e S}=\frac{E_{S}^{2}}{4 R_{S}}
$$

We find that

$$
\begin{array}{llll}
G_{e}>0 & \text { if } & R_{S} / R_{O}>0 \\
G_{e}<0 & \text { if } & R_{S} / R_{O}<0
\end{array}
$$

Observe that when $R_{S}>0$ and $R_{O}>0, G_{e}$ becomes the conventional available gain of the two-terminal-pair network.

The foregoing ideas lead to an extended definition, $F_{e}$, of the noise figure of a two-terminal-pair network ${ }^{1}$

$$
F_{e}=1+\frac{N_{e i}}{G_{e} k T_{0} \Delta f}
$$

where $N_{e i}$ is the exchangeable noise power at the network output when the source has a given (noiseless) impedance $Z_{S}$. Thus, $N_{e i}$ contains

${ }^{1}$ H. A. Haus and R. B. Adler, "An Extension of the Noise Figure Definition," Letter to the Editor, Proc. I.R.E., 45, 690 (1957). 
only the effect of internal network noise sources. The term $k T_{0} . \Delta f$ in Eq. 6.3 represents the available noise power from the input source impedance held at equilibrium at standard temperature $T_{0}$, provided this impedance is passive (that is, has a positive real part). In the case of a source impedance with a negative real part, $k T_{0} \Delta f$ merely represents an arbitrary but convenient normalization factor.

From Eqs. 6.1, 6.2, and 6.3, it is clear that

$$
\begin{array}{lll}
F_{e}-1>0 & \text { if } & R_{S}>0 \\
F_{e}-1<0 & \text { if } & R_{S}<0
\end{array}
$$

If $R_{S}>0$ and $R_{O}>0, F_{e}$ becomes the standard noise figure $F$.

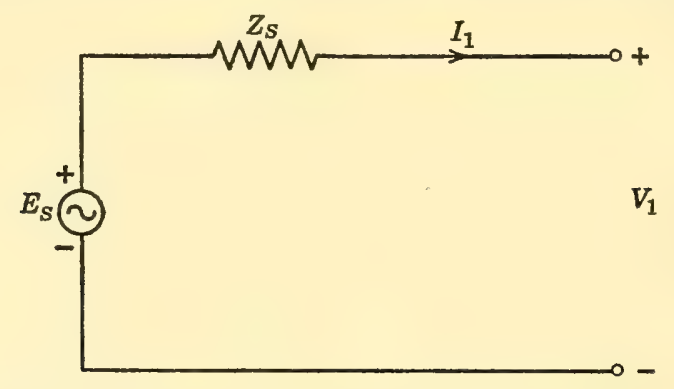

Fig. 6.1. Thévenin equivalent of two-terminal source.

\subsection{Matrix Formulation of Exchangeable Power and Noise Figure}

The parameter $p_{T}$ of Eq. 5.30, whose relation with the noise figure we are going to investigate, is expressed in matrix notation. Accordingly, to facilitate comparison, we must express the (extended) noise figure and the related parameters in the same manner.

Exchangeable Power. A linear two-terminal source of power can be represented by its Thévenin rms open-circuit voltage $E_{S}$, in series with its internal impedance $Z_{S}$. Let $V_{1}$ and $I_{1}$ be the terminal voltage and current of the source, respectively, as shown in Fig. 6.1, so that

$$
V_{1}+Z_{S} I_{1}=E_{S}
$$

To express Eq. 6.5 in matrix form suitable for cascade applications, we define column vectors (matrices) $\mathbf{v}$ and $\mathbf{x}$ as

$$
\mathbf{v}=\left[\begin{array}{l}
V_{1} \\
I_{1}
\end{array}\right] ; \quad \mathbf{x}=\left[\begin{array}{c}
1 \\
Z_{S} *
\end{array}\right]
$$

Then, for Eq. 6.5, we have

$$
\mathbf{x}^{\dagger} \mathbf{v}=E_{S}
$$


We note that Eq. 6.7 is the one-terminal-pair form of Eq. 5.1, where all the submatrices have become scalars.

Since multiplication of Eq. 6.7 by a constant $c$ does not alter it, we can, purely as a matter of form, always make a new column vector

$$
\mathbf{y}=\left[\begin{array}{l}
y_{1} \\
y_{2}
\end{array}\right]=c \mathbf{x}=\left[\begin{array}{c}
c \\
c Z_{S}^{*}
\end{array}\right]
$$

and a new scalar

$$
\gamma=c E_{S}
$$

so that the source equation (Eq. 6.7) becomes

$$
\mathbf{y}^{\dagger} \mathbf{v}=\gamma
$$

with

$$
\frac{y_{2}}{y_{1}}=Z_{S}{ }^{*}
$$

where $Z_{S}$ is still the internal impedance of the source. This formal multiplication feature of the source equation is helpful in interpreting the following analyses.

Now the exchangeable power $P_{e}$ of the source may be written in matrix form

$$
P_{e}=\frac{\left|E_{S}\right|^{2}}{2\left(Z_{S}+Z_{S}{ }^{*}\right)}=\frac{\left|E_{S}\right|^{2}}{2\left(\mathbf{x}^{\dagger} \mathbf{P x}\right)}=\frac{|\gamma|^{2}}{2\left(\mathbf{y}^{\dagger} \mathbf{P y}\right)}
$$

where the square "permutation" matrix $\mathbf{P}$ is the two-terminal-pair form of Eq. $5.26 b$.

$$
\mathbf{P}=\left[\begin{array}{ll}
0 & 1 \\
1 & 0
\end{array}\right]
$$

It has the properties $\mathbf{P}^{\dagger}=\mathbf{P}$ and $\mathbf{P}^{-1}=\mathbf{P}$ as indicated in Eqs. 5.27. The usefulness of the last expression in Eq. 6.10 lies in the fact that it can be written by inspection for any two-terminal source with a source equation in the form of Eq. 6.8 .

Exchangeable-Power Gain. Consider a linear source-free twoterminal-pair network, described by its general-circuit constants $A, B$, $C, D$, as shown in Fig. 6.2. If $\mathbf{v}$ is the "input" column vector defined by Eq. 6.6, and we let $\mathbf{u}$ be the "output" column vector,

$$
\mathbf{u}=\left[\begin{array}{l}
V_{2} \\
I_{2}
\end{array}\right]
$$

and $\mathbf{T}$ is the general-circuit matrix,

$$
\mathbf{T}=\left[\begin{array}{ll}
A & B \\
C & D
\end{array}\right]
$$


the network equations are expressed in matrix form as

$$
\mathbf{v}=\mathbf{T u}
$$

When the network of Fig. 6.2 is driven by the source of Fig. 6.1, the exchangeable output power $P_{e O}$ from terminal pair 2 can be obtained at

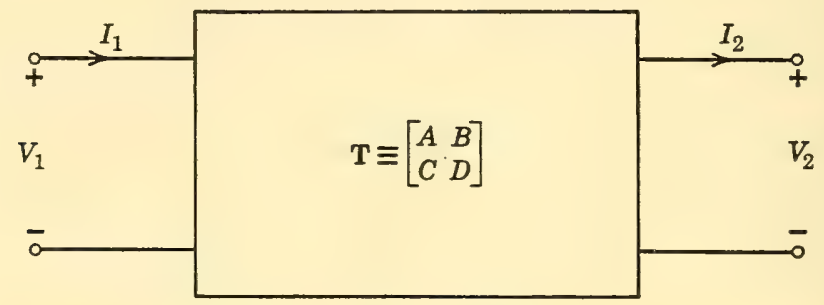

Fig. 6.2. The general-circuit-matrix representation of a source-free two-terminal-pair network.

once from a source equation in the form of Eq. 6.8 written with $\mathbf{u}$ as the voltage-current column variable. Multiplication of Eq. 6.13 by $\mathbf{y}^{\dagger}$, with the use of Eq. 6.8, yields this new relation,

$$
\left(\mathbf{y}^{\dagger} \mathbf{T}\right) \mathbf{u}=\gamma
$$

Accordingly, by applying the steps of Eqs. 6.8, 6.10, and 6.11 to Eq. 6.14, we find that

$$
P_{e O}=\frac{|\gamma|^{2}}{2 \mathbf{y}^{\dagger} \mathbf{T P T}^{\dagger} \mathbf{y}}
$$

and with Eq. 6.10, and Eq. 6.1 $a$ for the exchangeable gain, we have

$$
G_{e}=\frac{\mathbf{y}^{\dagger} \mathbf{P y}}{\mathbf{y}^{\dagger} \mathbf{T} \mathbf{P} \mathbf{T}^{\dagger} \mathbf{y}}
$$

Extended Noise Figure. To apply the matrix formulation of exchangeable power to the calculation of the (extended) noise figure of a two-terminal-pair noisy network, we still describe the network by its general-circuit constants, but we also allow for noise voltages or currents at the terminals in the absence of external sources. With reference to Fig. 6.3, the network equations for the dotted box ${ }^{2,3}$ would be

$$
\mathbf{v}=\mathbf{T} \mathbf{u}+\delta
$$

${ }^{2}$ A. G. Th. Becking, H. Groendijk, and K. S. Knol, "The Noise Factor of 4 Terminal Networks," Philips Research Repts. 10, 349-357 (1955).

${ }^{3}$ H. Rothe and W. Dahlke, "Theory of Noisy Four Poles," Proc. I.R.E., 44, 811-817 (1956). 
with $\delta$ a "noise column vector,"

$$
\delta=\left[\begin{array}{l}
E_{n 1} \\
I_{n 1}
\end{array}\right]
$$

Now Equation 6.17 can be rewritten as two relations:

$$
\begin{aligned}
\mathbf{v}^{\prime} & =\mathbf{T} \mathbf{u} \\
\mathbf{v} & =\mathbf{v}^{\prime}+\delta
\end{aligned}
$$

If we visualize $\mathbf{v}^{\prime}=\left[\begin{array}{c}V_{1}{ }^{\prime} \\ I_{1}{ }^{\prime}\end{array}\right]$ as referring simultaneously to the input terminals of a noise-free network $\mathbf{T}$ and the output terminals of a purenoise network $\delta$, the cascade division of the system is represented in Fig. 6.3. The noiseless part $\mathbf{T}$ does not affect the noise figure of the system. Thus, for noise-figure calculations, we need consider only the noise network $\delta$ driven by a source of internal impedance $Z_{S} \cdot{ }^{2,3}$

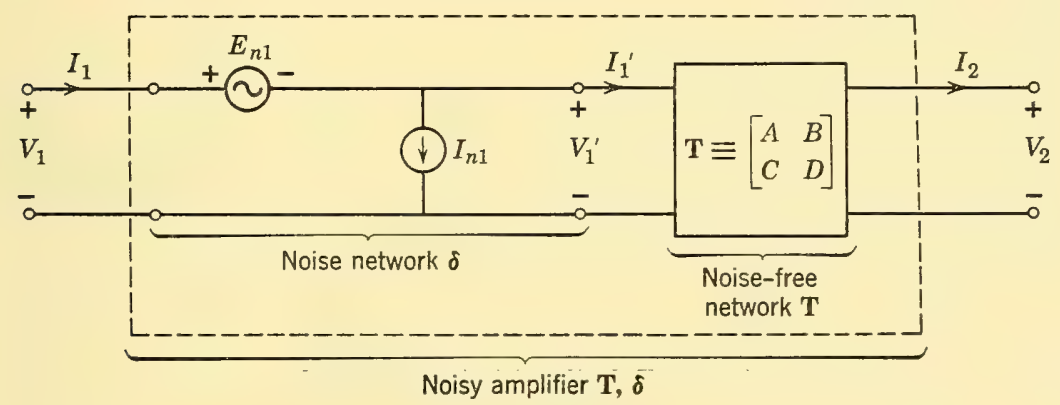

Fig. 6.3. The general-circuit-matrix representation of a linear two-terminal-pair network with internal sources.

The source equation appropriate to the right-hand terminals in Fig. 6.4 can be obtained from Eqs. $6.19 b$ and 6.8 with $E_{S}=0$.

$$
\mathbf{y}^{\dagger} \mathbf{v}^{\prime}=-\mathbf{y}^{\dagger} \delta
$$

Therefore, the output exchangeable power $N_{e i}$, produced by the internal noise only, is given by

$$
N_{e i}=\frac{\mathbf{y}^{\dagger} \overline{\delta \delta^{\dagger} \mathbf{y}}}{2 \mathbf{y}^{\dagger} \mathbf{P y}}
$$

and, since $G_{e}=1$ for this network, we have

$$
F_{e}-1=\frac{N_{e i}}{k T_{0} \Delta f}=\frac{\mathbf{y}^{\dagger} \overline{\delta \delta^{\dagger} \mathbf{y}}}{\mathbf{y}^{\dagger} \mathbf{P y}\left(2 k T_{0} \Delta f\right)}
$$




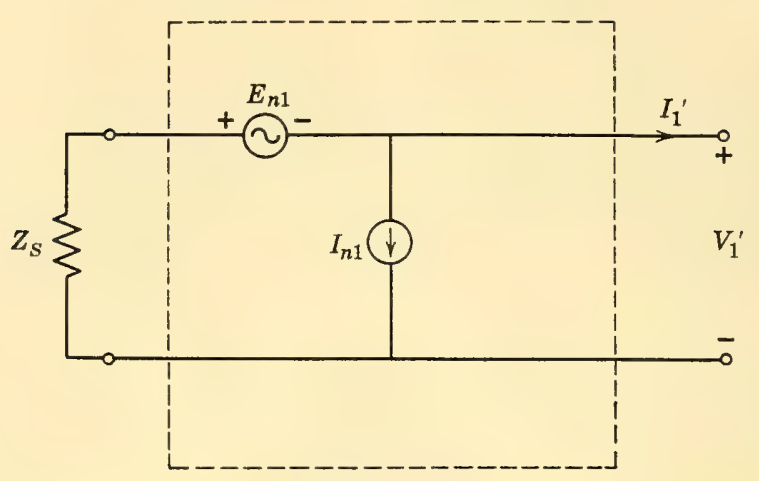

Noise network $\delta$

Fig. 6.4. Noise network of a linear noisy two-terminal-pair network in general-circuit-matrix representation.

\subsection{Noise Measure}

The "excess-noise figure," Eq. 6.22, has the same numerator as the noise parameter $p_{T}, \mathrm{Eq} .5 .30$, that we are trying to identify. The two equations differ only in the subtractive term $\mathbf{y}^{\dagger} \mathbf{T} \mathbf{P T}^{\dagger} \mathbf{y}$ in the denominator, aside from the multiplicative constant $k T_{0} \Delta f$. In order to facilitate the identification, we rewrite Eq. 5.30 in the form

$$
p_{T}=\frac{\mathbf{y}^{\dagger} \overline{\delta \delta^{\dagger} \mathbf{y}}}{2\left(\mathbf{y}^{\dagger} \mathbf{P y}\right)\left(1-\frac{\mathbf{y}^{\dagger} \mathbf{T P T}}{\mathbf{y}^{\dagger} \mathbf{P y}}\right)}
$$

From a comparison of Eq. 6.23 with Eqs. 6.22 and 6.16, it is obvious that

$$
\frac{p_{T}}{k T_{0} \Delta f}=\frac{F_{e}-1}{1-\frac{1}{G_{e}}}
$$

The expression on the right-hand side of Eq. 6.24 may now be identified as the noise parameter with the extremal properties corresponding to the network invariants. In the cases in which the extended definition of noise figure $F_{e}$ coincides with the conventional noise figure $F$ and the exchangeable gain $G_{e}$ is equal to the available gain $G$, the quantity in Eq. 6.24 is identical with the noise measure, Eq. 1.3. We shall now adopt this same name in the general case when $F_{e}$ and $G_{e}$ differ from the conventional $F$ and $G$, and denote this extended definition of noise measure 
by $M_{e}$ :

$$
M_{e} \equiv \frac{F_{e}-1}{1-\frac{1}{G_{e}}}=\frac{\mathbf{y}^{\dagger} \overline{\delta \delta^{\dagger} \mathbf{y}}}{\mathbf{y}^{\dagger}\left(\mathbf{P}-\mathbf{T P T}^{\dagger}\right) \mathbf{y}} \frac{1}{\left(2 k T_{0} \Delta f\right)}
$$

According to Eq. 6.9, the column vector $\mathbf{y}$ is determined, within a constant multiplier, by the source impedance $Z_{S}$ at which the noise measure $M_{e}$ of Eq. 6.25 is achieved.

In the discussion of the noise performance of amplifiers, it turns out to be important to bear in mind the algebraic sign that $M_{e}$ assumes under various physical conditions. These are summarized in Table 6.1.

Table 6.1. Algebraic Signs of Exchangeable Gain AND DeRIVEd Quantities

\begin{tabular}{cccccc}
$R_{S}$ & $R_{0}$ & $G_{e}$ & $F_{e}-1$ & $\left|G_{e}\right|$ & $M_{e}$ \\
\hline$>0$ & $>0$ & $>0$ & $>0$ & $>1$ & $>0$ \\
$>0$ & $>0$ & $>0$ & $>0$ & $<1$ & $<0$ \\
$>0$ & $<0$ & $<0$ & $>0$ & $\gtrless 1$ & $>0$ \\
$<0$ & $>0$ & $<0$ & $<0$ & $\gtrless 1$ & $<0$ \\
$<0$ & $<0$ & $>0$ & $<0$ & $>1$ & $<0$ \\
$<0$ & $<0$ & $>0$ & $<0$ & $<1$ & $>0$
\end{tabular}

With reference to Table 6.1, it should be pointed out that for $R_{S}>0$, $\left(F_{e}-1\right)>0$. When $R_{S}>0$, conventional available gain greater than 1 occurs in only two ways:

$$
\begin{aligned}
& \text { (a) } G_{e}>1 \\
& \text { (b) } G_{e}<0
\end{aligned}
$$

Case $a$ holds whenever the amplifier has an output impedance with positive real part and an available (or exchangeable) gain greater than 1. Case $b$ corresponds to an amplifier with an output impedance having a negative real part. Such an amplifier has an infinite available gain in the conventional sense. In both cases $M_{e}$ is found to be greater than zero.

In the succeeding portions of our work, we shall restrict our consideration of amplifier performance to cases in which the source has an internal impedance with a positive real part. This is the only case of practical interest. Indeed, any amplifying system, however complicated, is essentially a two-terminal-pair network driven by a signal transducer with a positive real part to its output impedance (for example, antenna, microphone, and so forth).

\subsection{Allowed Ranges of Values of the Noise Measure}

Let us consider a noisy two-terminal-pair network with the noise column matrix $\delta$ and the matrix of general circuit constants $\mathbf{T}$. We 
suppose that the input terminal pair of this network is connected to a source with the internal impedance $Z_{S}$. The noise measure of the network as measured at its output terminal pairs is then given by Eq. 6.25, where the column vector $\mathbf{y}$ satisfies the relation

$$
\frac{y_{2}}{y_{1}}=Z_{S}^{*}
$$

Next, we suppose that the original network is imbedded in a four-terminal-pair, lossless network (Fig. 6.5) before we connect it to the source. A new network results, with the noise column vector $\delta^{\prime}$ and the matrix $\mathbf{T}^{\prime}$. If one of the terminal pairs of the resulting network is connected to the same source, a new noise measure $M_{e}^{\prime}$ is observed at the other terminal pair:

$$
M_{e}^{\prime}=\frac{\mathbf{y}^{\dagger} \overline{\delta^{\prime} \delta^{\prime \dagger} \mathbf{y}}}{\mathbf{y}^{\dagger}\left(\mathbf{P}-\mathbf{T}^{\prime} \mathbf{P} \mathbf{T}^{\prime \dagger}\right) \mathbf{y}} \frac{1}{2 k T_{0} \Delta f}
$$

We shall now determine how the primed matrices in Eq. 6.26 are related to the unprimed matrices of the original network. First, from Eqs. 3.3 and 3.6 we know that after a lossless transformation

$$
\overline{\mathbf{E}^{\prime} \mathbf{E}^{\prime \dagger}}=\tau^{\dagger} \overline{\mathbf{E E}^{\dagger} \tau}
$$

and

$$
\mathbf{Z}^{\prime}+\mathbf{Z}^{\prime \dagger}=\tau^{\dagger}\left(\mathbf{Z}+\mathbf{Z}^{\dagger}\right) \boldsymbol{\tau}
$$

However, from Eqs. 5.22, 5.23, and 5.28 we have

$$
\begin{aligned}
\overline{\delta^{\prime} \delta^{\prime \dagger}} & =\mathbf{M}^{\prime} \overline{\mathbf{E}^{\prime} \mathbf{E}^{\prime \dagger}} \mathbf{M}^{\prime \dagger}=\mathbf{M}^{\prime} \boldsymbol{\tau}^{\dagger} \overline{\mathbf{E} \mathbf{E}^{\dagger}} \boldsymbol{\tau} \mathbf{M}^{\prime \dagger} \\
& =\left(\mathbf{M}^{\prime} \boldsymbol{\tau}^{\dagger} \mathbf{M}^{-1}\right) \overline{\delta \delta^{\dagger}}\left(\mathbf{M}^{\prime} \boldsymbol{\tau}^{\dagger} \mathbf{M}^{-1}\right)^{\dagger} \\
& =\mathbf{C}^{\dagger} \overline{\delta \delta^{\dagger} \mathbf{C}}
\end{aligned}
$$

and

$$
\begin{aligned}
\left(\mathbf{P}-\mathbf{T}^{\prime} \mathbf{P} \mathbf{T}^{\prime \dagger}\right) & =\mathbf{M}^{\prime}\left(\mathbf{Z}^{\prime}+\mathbf{Z}^{\prime \dagger}\right) \mathbf{M}^{\dagger \dagger}=-\mathbf{M}^{\prime} \boldsymbol{\tau}^{\dagger}\left(\mathbf{Z}+\mathbf{Z}^{\dagger}\right) \boldsymbol{\tau} \mathbf{M}^{\prime \dagger} \\
& =\left(\mathbf{M}^{\prime} \boldsymbol{\tau}^{\dagger} \mathbf{M}^{-1}\right)\left(\mathbf{P}-\mathbf{T} \mathbf{P} \mathbf{T}^{\dagger}\right)\left(\mathbf{M}^{\prime} \boldsymbol{\tau}^{\dagger} \mathbf{M}^{-1}\right)^{\dagger} \\
& =\mathbf{C}^{\dagger}\left(\mathbf{P}-\mathbf{T} \mathbf{P} \mathbf{T}^{\dagger}\right) \mathbf{C}
\end{aligned}
$$

where

$$
\mathbf{C}^{\dagger}=\mathbf{M}^{\prime} \boldsymbol{\tau}^{\dagger} \mathbf{M}^{-1}
$$

The matrix $\mathbf{C}$ involved in the colinear transformations of $\overline{\delta \delta^{\dagger}}$ and $\mathbf{P}-\mathbf{T P T}^{\dagger}$ can be adjusted arbitrarily by arbitrary changes in the imbedding network, on account of the matrix $\tau$ of the lossless transformation that appears in $\mathbf{C}$.

Introducing the explicit transformations, Eqs. 6.27 through 6.29, into 

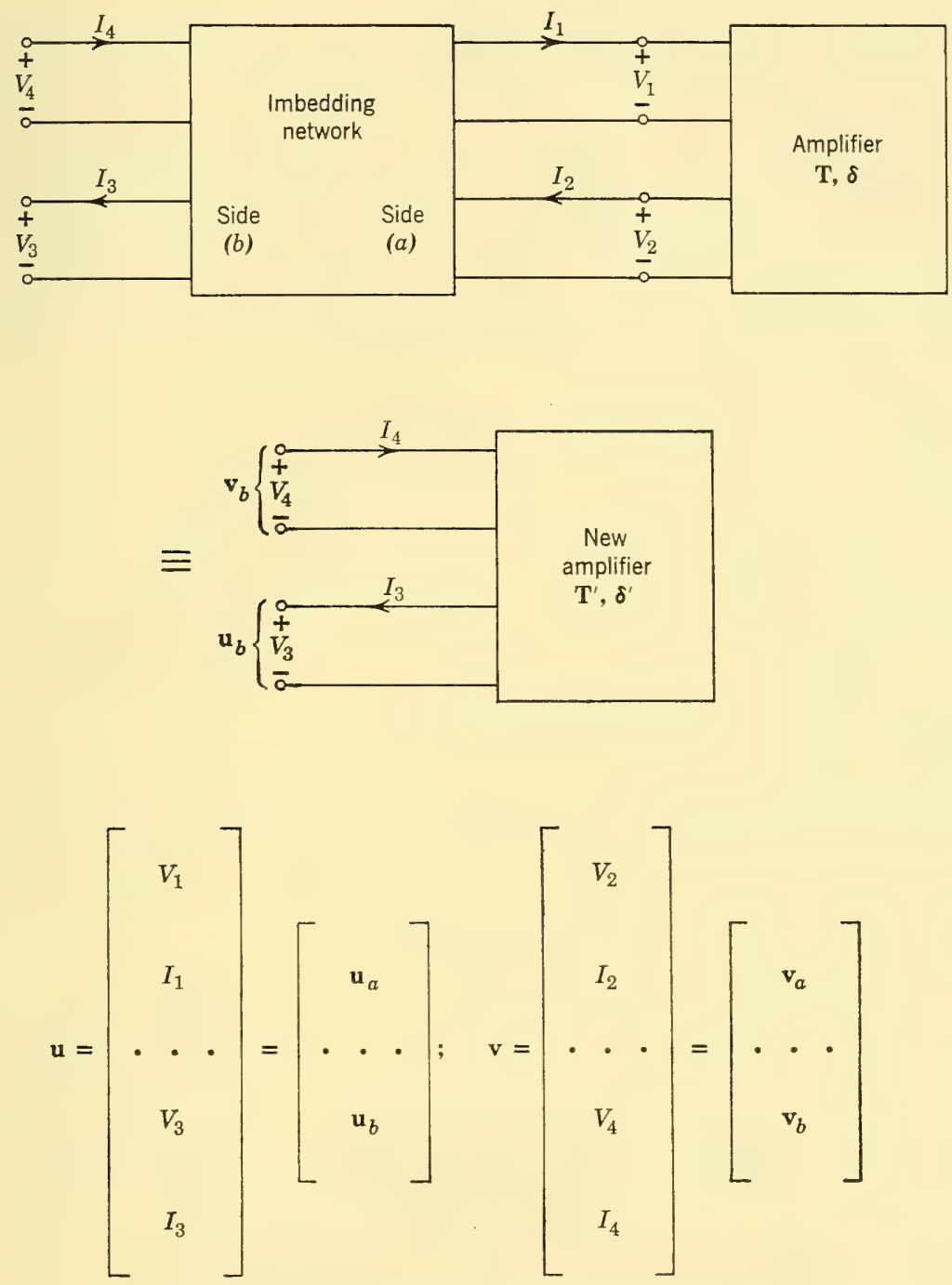

Fig. 6.5. Imbedding of two-terminal-pair amplifier.

the noise-measure expression, Eq. 6.26, we have

$$
M_{e}{ }^{\prime}=\frac{(\mathbf{C y})^{\dagger} \overline{\delta \delta^{\dagger}}(\mathbf{C y})}{(\mathbf{C y})^{\dagger}\left(\mathbf{P}-\mathbf{T P T}^{\dagger}\right)(\mathbf{C y})} \frac{1}{2 k T_{0} \Delta f}
$$

Through variations of the imbedding network, the column vector $\mathbf{C y}$ can be varied arbitrarily. In this manner $M_{e}^{\prime}$ can be varied over its entire allowed ranges, which are limited by the two eigenvalues $\lambda_{1}$ and $\lambda_{2}$ 
of $\mathbf{N}_{T}$. By making use of Table 5.1 and Eq. 5.28 in the special case of a two-terminal-pair network, we see that the following three cases have to be distinguished:

1. $\mathbf{P}-\mathbf{T P T}^{\dagger}$ is negative definite; $\mathbf{T}$ is the general circuit matrix of a passive network, $M_{e}^{\prime}<0$.

2. $\mathbf{P}-\mathbf{T P T}^{\dagger}$ is positive definite; $\mathbf{T}$ is the general circuit matrix of a negative-resistance network, $M_{e}{ }^{\prime}>0$.

3. $\mathbf{P}-$ TPT $^{\dagger}$ is indefinite; $\mathbf{T}$ is the general circuit matrix of a network capable of absorption, as well as delivery of power, $M_{e}{ }^{\prime} \gtrless 0$.

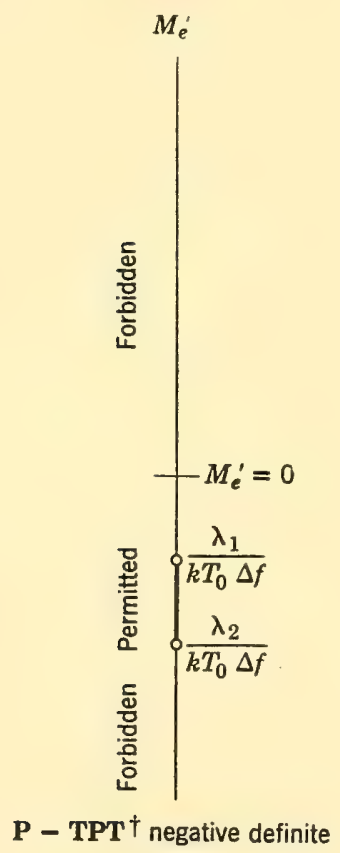

(a)

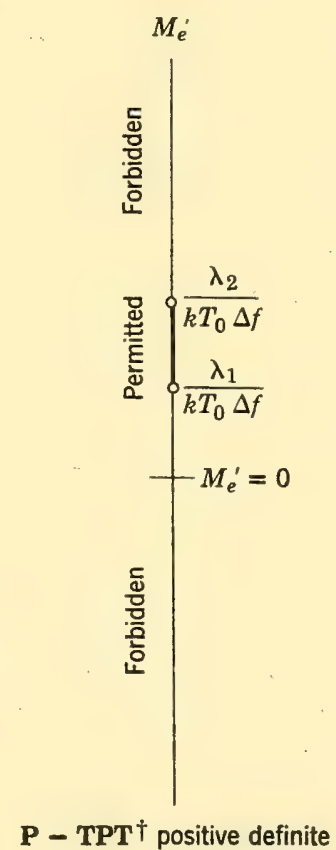

(b)

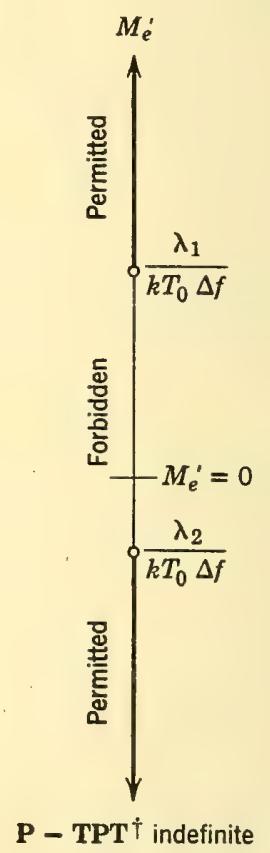

(c)

Fig. 6.6. Schematic diagram of permitted values of $M_{e}^{\prime}$ for two-terminal-pair networks.

When $\mathbf{C}$ in Eq. 6.30 is varied through all possible values, $M_{e}{ }^{\prime}$ reaches two extrema, which are the two eigenvalues of the characteristic-noise matrix $\mathbf{N}_{T}$ divided by $k T_{0} \Delta f$. Now, we have pointed out that in practical situations amplifiers are driven from sources having an internal impedance with positive real part. According to Table 6.1, the noise measure is positive when $R_{S}>0$ in all cases except $R_{O}>0,0<G_{e}<1$. This case does not correspond to an amplifier. Hence we shall be interested in achieving only positive values of $M_{e}{ }^{\prime}$, which occur in Cases $b$ and $c$ of Fig. 6.6. These cases both have an available gain, $G$, in the con- 
ventional sense, greater than unity $\left(G=G_{e}\right.$, if $G_{e}>1 ; G=\infty$, if $\left.G_{e}<0\right)$.

We observe from Eq. 6.30 that the numerator is never negative. Therefore, changes in sign of $M_{e}{ }^{\prime}$ occur with those of the denominator. In Case $c$, which includes most conventional amplifiers, $M_{e}^{\prime}$ changes sign only at a zero of the denominator. Thus, the values of $M_{e}{ }^{\prime}$ cannot lie between $\lambda_{1} /\left(k T_{0} \Delta f\right)$ and $\lambda_{2} /\left(k T_{0} \Delta f\right)$.

The two cases of interest, Cases $b$ and $c$ of Fig. 6.6, have a least positive eigenvalue of $\mathbf{N}$ which we call $\lambda_{1}>0$. We have therefore proved the following theorem:

Consider the set of lossless transformations that carry a two-terminalpair amplifier into a new two-terminal-pair amplifier with a conventional available gain $G$ greater than 1 . When driven from a source that has an internal impedance with positive real part, the noise measure of the transformed amplifier cannot be less than $\lambda_{1} /\left(k T_{0} \Delta f\right)$, where $\lambda_{1}$ is the smallest positive eigenvalue of the characteristic-noise matrix of the original amplifier.

The fact that $M_{e}^{\prime} \geq \lambda_{1} /\left(k T_{0} \Delta f\right)$ also puts a lower limit upon the excess-noise figure. Suppose that the amplifier is imbedded in a lossless network and then connected to a source with an internal impedance having a positive real part. Let the resulting exchangeable power gain be $G_{e}$. Then, the excess-noise figure of the resulting amplifier has to fulfill the inequality

$$
F_{e}-1 \geq \frac{\lambda_{1}}{k T_{0} \Delta f}\left(1-\frac{1}{G_{e}}\right)
$$

Consequently, an amplifier has a definite lower limit imposed on its excess-noise figure, and this limit depends upon the exchangeable-power gain achieved in the particular connection.

If $G_{e}>0$, that is, the output impedance of the amplifier has a positive real part, the excess-noise figure can be less than $\lambda_{1} /\left(k T_{0} \Delta f\right)$ only to the extent of the gain-dependent factor $\left(1-1 / G_{e}\right)$.

If $G_{e}<0$, that is, the output impedance has a negative real part, the lower limit to the excess-noise figure is higher than $\lambda_{1} /\left(k T_{0} \Delta f\right)$ by $\left(1+\left|1 / G_{e}\right|\right)$.

Thus, if two amplifiers with the same eigenvalue $\lambda_{1}$ of their characteristicnoise matrix are driven with a positive source impedance, one of which has a positive output impedance, the other a negative one, then the minimum excess-noise figure of the latter cannot be less than that of the former.

Equation 6.31 has established a gain-dependent lower bound for the excess-noise figure achievable with lossless imbeddings of a given amplifier. At large values of $\left|G_{e}\right|$, the excess-noise figure is evidently equal to the 
noise measure. Large values of $\left|G_{e}\right|$ must correspond to large values of conventional available gain. Therefore, the excess-noise figure at large conventional available gain is limited to values greater than, or equal to, $\lambda_{1} / k T_{0} \Delta f$ under the most general lossless external network operations on the amplifier. These include, for example, lossless feedback, input mismatch, and so forth. On the supposition that the noise figure at large conventional available gain is a meaningful measure of the quality of amplifier noise performance, the minimum positive value of the noise measure,

$$
M_{e, \text { opt }}=\frac{\lambda_{1}}{k T_{0} \Delta f}
$$

is a significant noise parameter of the amplifier. The significance of $M_{e, \text { opt }}$ will be further enhanced by the proofs, given in the remaining sections, of the following statements:

1. The lower bound $M_{e, \text { opt }}$ on the noise measure of an amplifier can actually be achieved by appropriate imbedding. Moreover, this is accomplished in such a way that subsequent cascading of identical units realizes $M_{e, \text { opt }}$ as the excess-noise figure at arbitrarily high gain.

2. An arbitrary passive interconnection of independently noisy amplifiers with different values of $M_{e, \text { opt }}$ cannot yield a new two-terminal-pair amplifier with an excess-noise figure at large conventional available gain lower than $M_{e, \text { opt }}$ of the best component amplifier (the one with the smallest value of $M_{e, \text { opt }}$ ).

3. The use of passive dissipative imbedding networks for a given amplifier driven with a positive source impedance cannot achieve a positive noise measure less than its $M_{e, \text { opt }}$.

We shall take up statements 2 and 3 first.

\subsection{Arbitrary Passive Interconnection of Amplifiers}

To prove statements 2 and 3 of Sec. 6.4, we begin by considering a general lossless interconnection of $n$ independently noisy amplifiers, as shown in Fig. 6.7. A 2n-terminal-pair network results. By opencircuiting all but two of the resulting terminal pairs, we obtain the most general two-terminal-pair amplifier obtainable from the original ones by lossless interconnection. In Sec. 4.2 we have developed the general theory of such an imbedding and reduction of terminal pairs. Indeed, Fig. 4.3 includes the situation of Fig. 6.7. We know that the eigenvalues of the characteristic-noise matrix of the network lie between the most extreme eigenvalues of the characteristic-noise matrices of the original $n$ amplifiers. Therefore the lowest positive eigenvalue of the 
reduced network cannot be less than the lowest positive eigenvalue in the original lot. Accordingly, the optimum noise measure $M_{e, \text { opt }}$ of the reduced network cannot be less than that of the best of the original amplifiers.

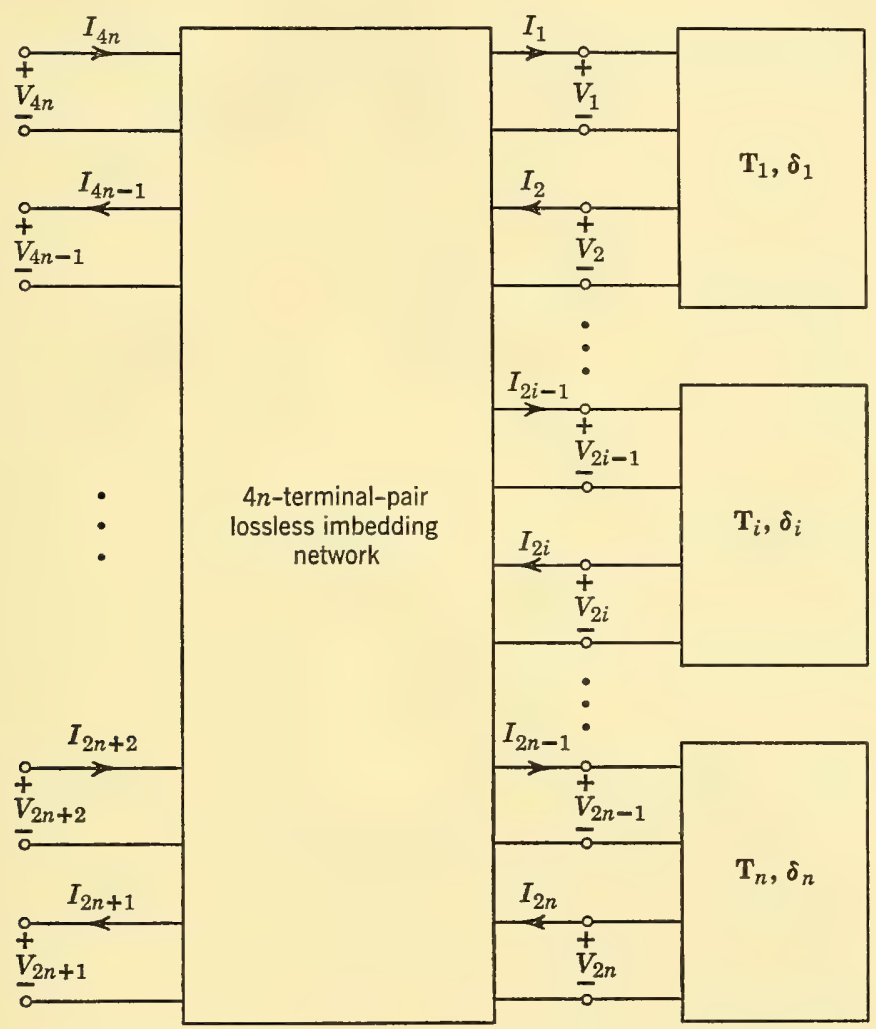

Fig. 6.7. Lossless interconnection of several amplifiers.

We shall now extend our proof to cover passive interconnections of amplifiers. We again consider $n$ amplifiers but shall now imbed them into a dissipative $4 n$-terminal-pair network in the configuration of Fig. 6.7. Suppose, for a moment, that we disconnect the amplifiers and that we throw the imbedding network into its canonical form, as discussed in Sec. 4.1. This is accomplished by a lossless network transformation, and results in an arrangement like Fig. 4.2 but with $4 n$ separate resistances and uncorrelated noise generators. These resistances are arranged in the way shown at the bottom of Fig. 6.8. If we now apply to this canonical form the lossless network transformation inverse to the one 
that produced the canonical form, we obtain a $4 n$-terminal-pair network which has the same terminal behavior as the original dissipative imbedding network. The resulting network is shown in Fig. 6.8. It has $2 n$ available terminal pairs. The $4 n$ resistances can be comprised in a $4 n$-terminal-pair network with a characteristic-noise matrix, the eigen-

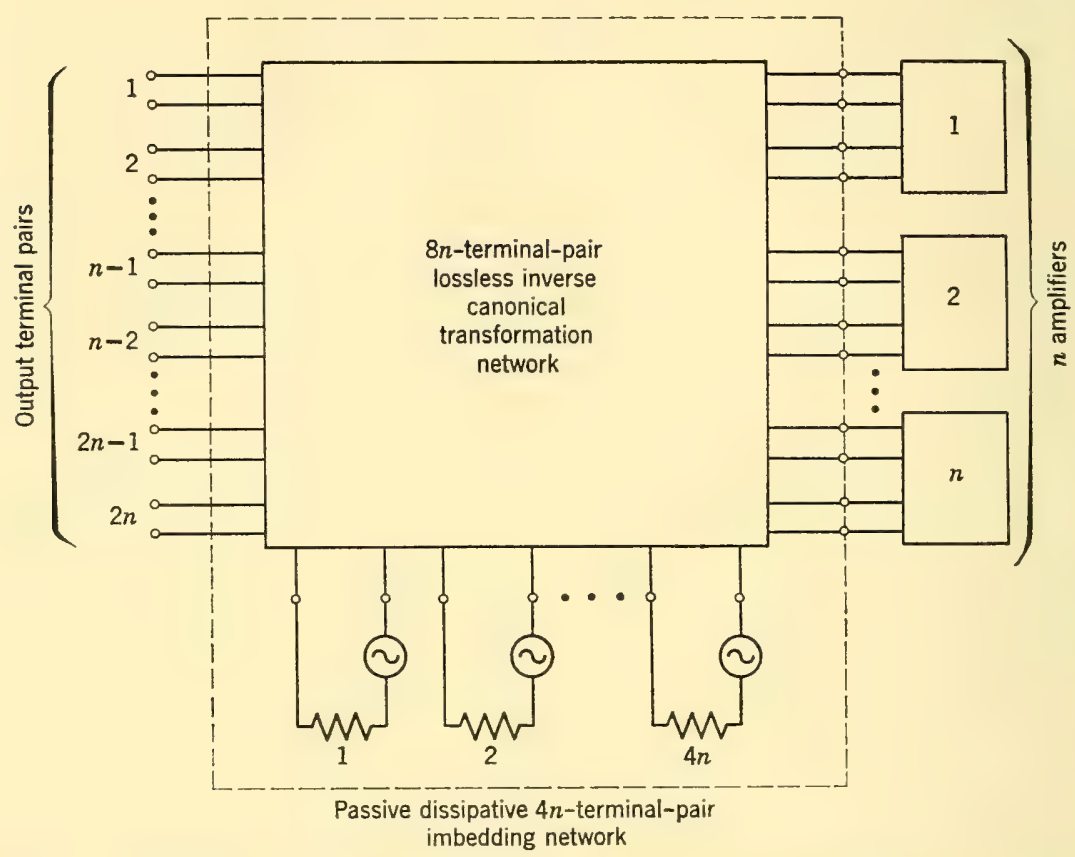

Fig. 6.8. Imbedding of $n$ two-terminal-pair amplifiers in lossy $4 n$-terminal-pair network.

values of which are all negative (Sec. 3.3). The $n$ amplifiers can be grouped correspondingly into a $2 n$-terminal-pair network with a characteristic-noise matrix that has (in general, positive and negative) eigenvalues equal to those of the characteristic-noise matrices of the original amplifiers. The network operation in Fig. 6.8 is obtained from that corresponding to Fig. 4.3 by a subsequent reduction from $6 n$ to $2 n$ output terminal pairs. Again, the least positive eigenvalue of the characteristicnoise matrix is not less than that of the best amplifier. Thus, we may state the following theorem:

Consider a general lossless or passive dissipative interconnection of an arbitrary number of different and independently noisy two-terminal-pair amplifiers that results in a two-terminal-pair network. When the resulting two-terminal-pair network is driven from a source that has an internal 
impedance with positive real part, its optimum noise measure $M_{e, \text { opt }}$ cannot be less than that of the best amplifier, that is, the amplifier with the least positive eigenvalue of its characteristic-noise matrix.

Statement 3 of Section 6.4 is an immediate corollary of the previous theorem; that is,

Passive dissipative imbedding of a given two-terminal-pair amplifier cannot reduce its noise measure to a positive value below $M_{e, \text { opt }}$, provided that the source impedance has a positive real part. 


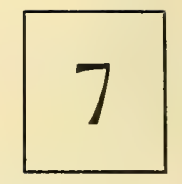

\section{Network Realization}

\section{of Optimum Amplifier \\ Noise Performance}

In Chap. 6 we have shown that the excess-noise figure of an amplifier with a high gain cannot be less than $M_{e, \text { opt }}$,

$$
M_{e, \mathrm{opt}}=\frac{\lambda_{1}}{k T_{0} \Delta f}
$$

where $\lambda_{1}$ is the smallest positive eigenvalue of the characteristic-noise matrix. We also showed that an arbitrary lossless or passive interconnection of two-terminal-pair amplifiers, which leads to a new two-terminalpair amplifier, yields an excess-noise figure at high gain that is higher than, or at best equal to, the $M_{e, \text { opt }}$ of the best amplifier used in the interconnection. These proofs established the quantity $M_{e, \text { opt }}$ as a lower bound on the noise performance of a two-terminal-pair amplifier.

In this chapter we shall show that the lower bound $M_{e, \text { opt }}$ on the excessnoise figure at high gain can always be realized. Specifically, we shall show that the minimum positive noise measure $M_{e, \text { opt }}$ of any twoterminal-pair amplifier can be achieved by suitable external network operations, which, however, do not usually result in an amplifier with a high gain. Nevertheless, the source impedance, and the amplifier output impedance with the source connected, will always have positive real parts in the realizations of $M_{e, \text { opt }}$ presented. It follows that an amplifier with an arbitrarily high gain can be constructed by cascading identical, optimized amplifiers that have appropriate impedance-transformation networks between the stages. By an adjustment of the transformation networks, the optimum noise measure of the cascade can be made equal to the $M_{e, \text { opt }}$ of the individual amplifiers in the cascade, as explained in 
Chap. 1. The excess-noise figure of the high-gain cascade is equal to the optimum noise measure of the cascade, and thus in turn equal to $M_{e, \text { opt }}$ of the individual amplifiers. This arrangement therefore accomplishes the realization of the lower limit of the excess-noise figure at high gain.

Since $M_{e, \text { opt }}$ of any given amplifier determines the lowest (excess-) noise figure that can be achieved at high gain with the amplifier, either singly or in interconnection with other amplifiers of the same type, we may conclude from the criterion chosen in Chap. 1 that $M_{e, \text { opt }}$ is an absolute measure of the quality of noise performance of a given amplifier.

\subsection{Classification of Two-Terminal-Pair Amplifiers}

The noise-performance optimization problem is solved conveniently by referring to a detailed classification of nonpassive two-terminal-pair networks (that is, amplifiers). Mason ${ }^{1}$ has shown that every such network can be reduced by lossless reciprocal imbedding to one of the three basic types shown in Fig. 7.1. His classification is based primarily upon the range of values of the unilateral gain $U$

$$
U=\frac{\left|Z_{21}-Z_{12}\right|^{2}}{4\left(R_{11} R_{22}-R_{12} R_{21}\right)}
$$

where the $R$ 's are the real parts of the impedance-matrix elements. Since the numerical value of $U$ is invariant to lossless reciprocal transformations, ${ }^{1}$ none of the three types can be carried into any other type by such transformations.

The first type (Fig. 7.1a), with $U>1$, is by far the most common. The majority of vacuum-tube and transistor amplifiers belong to this class. The second type (Fig. 7.1b), with $U<0$, is less common. It does, however, share one important property with the first; namely, both have

$$
\operatorname{det}\left(\mathbf{P}-\mathbf{T P}^{\dagger}\right)<0
$$

which means that they can absorb as well as deliver power. It is perhaps not surprising, therefore, that with lossless nonreciprocal transformations amplifiers of the type of Fig. $7.1 b$ can be carried into the form shown in Fig. 7.1a. This we now show.

The network of Fig. $7.1 b$ has a unilateral gain

$$
U=-|u|^{2}<0 \text {. }
$$

By connecting the network in series with a lossless gyrator with the

${ }^{1}$ S. J. Mason, "Power Gain in Feedback Amplifiers," Trans. IRE, Professional Group on Circuit Theory, CT-1, No. 2, 20 (1954). 


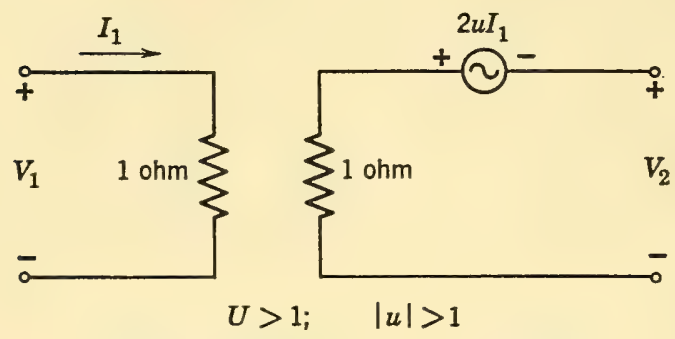

$$
\operatorname{Det}\left(\mathbf{P}-\mathbf{T P T}^{\dagger}\right)=\frac{1}{|u|^{2}}-1<0 \text {; indefinite }
$$

(a)

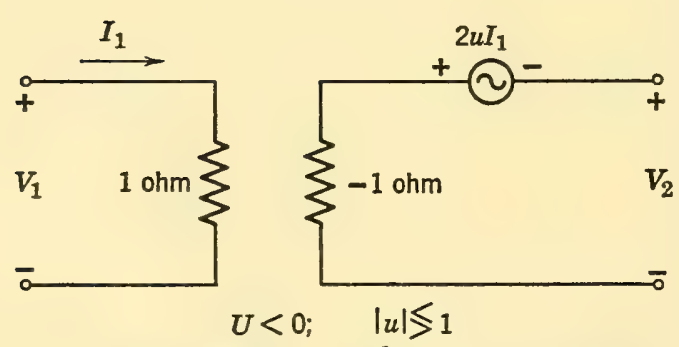

$$
\operatorname{Det}\left(\mathbf{P}-\mathbf{T P T}^{\dagger}\right)=-\left(\frac{1}{|u|^{2}}+1\right)<0 \text {; indefinite }
$$

(b)

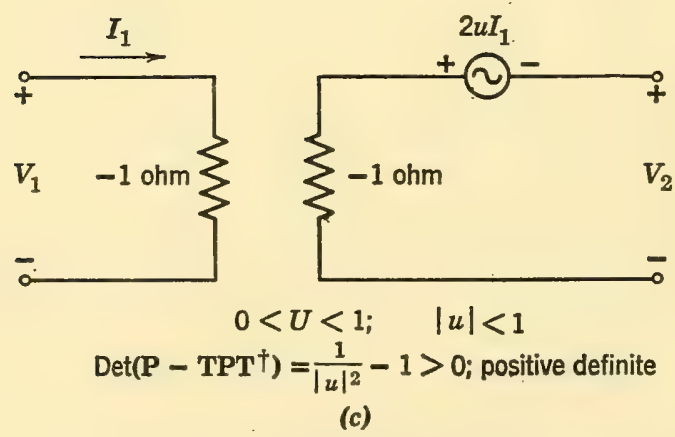

Fig. 7.1. Classes of amplifiers. 
impedance matrix

$$
\mathbf{Z}=\left[\begin{array}{rr}
0 & r \\
-r & 0
\end{array}\right]
$$

where $r$ is real, we obtain, for the unilateral gain (Eq. 7.1) of the combined network,

$$
U=\frac{[\operatorname{Re}(u)+r]^{2}+\operatorname{Im}^{2}(u)}{-1+r^{2}+2 r \operatorname{Re}(u)}
$$

In Eq. 7.4, $U$ can always be made positive and greater than unity by choosing

$$
r>+\sqrt{[\operatorname{Re}(u)]^{2}+1}-\operatorname{Re}(u)
$$

Thus, the network of Fig. $7.1 \mathrm{~b}$ can always be given a unilateral gain that is positive and greater than unity. Then, according to Mason's work, ${ }^{1}$ it can be unilateralized and brought into the form of Fig. 7.1 $a$.

The amplifier in Fig. 7.1c, however, has

$$
\operatorname{det}\left(\mathbf{P}-\mathbf{T P} \mathbf{T}^{\dagger}\right)>0
$$

and cannot, under any terminal condition, absorb power. Obviously it cannot be reduced to any of the other forms by any lossless transformation whatsoever. It is a "negative-resistance" amplifier.

Recalling that the optimum noise measure is not changed by any lossless transformation, we conclude that noise-performance optimization of amplifiers need only be carried out on networks of the specific forms Figs. $7.1 a$ and $7.1 c$, and such a procedure will be sufficient to include all nonpassive cases.

\subsection{Optimization of Amplifier, Indefinite Case}

We imagine that the given amplifier with $\operatorname{det}\left(\mathbf{P}-\mathbf{T P T}^{\dagger}\right)<0$ is initially in, or is reduced to, the form of Fig. 7.1a. We shall show that $M_{e, o p t}$ can be realized for this circuit by suitable input mismatch, retaining positive source impedance.

The general circuit matrix of the amplifier in Fig. $7.1 a$ is

$$
\mathbf{T}=-\frac{1}{2 u}\left[\begin{array}{ll}
1 & 1 \\
1 & 1
\end{array}\right]
$$

and the noise column matrix (elements not shown in Fig. 7.1a) is

$$
\delta=\left[\begin{array}{c}
E_{n 1} \\
I_{n 1}
\end{array}\right]
$$


The characteristic-noise matrix of this amplifier can be computed to be

$$
\begin{aligned}
\mathbf{N} & =\frac{1}{2}\left(\mathbf{P}-\mathbf{T P T}^{\dagger}\right)^{-1} \overline{\delta \delta^{\dagger}} \\
& =\frac{2|u|^{2}-1}{4\left(|u|^{2}-1\right)}\left[\begin{array}{l:l}
\frac{\overline{\left.E_{n 1}\right|^{2}}}{2\left|u^{2}\right|-1}+\overline{E_{n 1}{ }^{*} I_{n 1}} & \frac{\overline{E_{n 1} I_{n 1}{ }^{*}}}{2|u|^{2}-1}+\overline{\left.I_{n 1}\right|^{2}} \\
\hdashline \overline{\left.E_{n 1}\right|^{2}}+\frac{\overline{E_{n 1} I_{n 1}}}{2|u|^{2}-1} & \overline{E_{n 1} I_{n 1}{ }^{*}}+\frac{\mid \overline{\left.I_{n 1}\right|^{2}}}{2|u|^{2}-1}
\end{array}\right]
\end{aligned}
$$

The amplifier noise measure in matrix form is given by Eq. 6.25:

$$
M_{e}=\frac{\mathbf{y}^{\dagger} \overline{\delta \delta^{\dagger} \mathbf{y}}}{\mathbf{y}^{\dagger}\left(\mathbf{P}-\mathbf{T P T}^{\dagger}\right) \mathbf{y}} \frac{1}{2 k T_{0} \Delta f}
$$

where $\mathbf{y}$ is a column vector fulfilling the condition:

$$
\frac{y_{2}}{y_{1}}=Z_{S} *
$$

in which $Z_{S}$ is the source impedance.

The noise measure of $\mathrm{Eq}$. 6.25 reaches its least positive value when the vector $\mathbf{y}$ is equal to that eigenvector $\mathbf{y}^{(1)}$ of $\mathbf{N}$ which pertains to the positive eigenvalue of $\mathbf{N}$. The vector $\mathbf{y}$ is adjusted by an adjustment of the source impedance $Z_{S}$. Hence, the noise measure can be optimized by a lossless impedance-matching network at the input of the amplifier if the actual source impedance with a positive real part can be transformed into the value $Z_{S}{ }^{(1)}$ prescribed by the eigenvector $y^{(1)}$,

$$
Z_{S}^{(1)}=\left(\frac{y_{2}^{(1)}}{y_{1}^{(1)}}\right)^{*}
$$

Thus, if the noise measure is to be optimized by a lossless mismatching network, it is necessary and sufficient that

$$
\operatorname{Re}\left(\frac{y_{2}^{(1)}}{y_{1}^{(1)}}\right)=\operatorname{Re}\left(Z_{S}^{(1)}\right)>0
$$

The proof that the inequality (Eq. 7.10) is fulfilled for $|u|>1$ will now be carried out.

The proof is greatly facilitated if we use a wave formalism rather than the voltage-current formalism. We assume that transmission lines of 1-ohm characteristic impedance are connected to the amplifiers. The incident waves $a_{1}$ and $a_{2}$ and the reflected waves $b_{1}$ and $b_{2}$ on these 
transmission lines are related to the terminal voltages and currents by

$$
\begin{array}{ll}
a_{1}=\frac{1}{2}\left(V_{1}+I_{1}\right) ; & b_{2}=\frac{1}{2}\left(V_{2}+I_{2}\right) \\
b_{1}=\frac{1}{2}\left(V_{1}-I_{1}\right) ; & a_{2}=\frac{1}{2}\left(V_{2}-I_{2}\right)
\end{array}
$$

These transformations are conveniently summarized in matrix form. We define the matrix

$$
\mathbf{R}=\frac{1}{2}\left[\begin{array}{rr}
1 & 1 \\
1 & -1
\end{array}\right]
$$

We then have

$$
\begin{aligned}
\mathbf{v}^{\prime} & =\left[\begin{array}{l}
a_{1} \\
b_{1}
\end{array}\right]=\mathbf{R}\left[\begin{array}{l}
V_{1} \\
I_{1}
\end{array}\right]=\mathbf{R v} \\
\mathbf{u}^{\prime} & =\left[\begin{array}{l}
b_{2} \\
a_{2}
\end{array}\right]=\mathbf{R}\left[\begin{array}{l}
V_{2} \\
I_{2}
\end{array}\right]=\mathbf{R u}
\end{aligned}
$$

The general-circuit-parameter representation of the network is

$$
\mathbf{v}-\mathbf{T u}=\delta
$$

The matrix equation for the new choice of variables $\mathbf{v}^{\prime}$ and $\mathbf{u}^{\prime}$ has the general form

$$
\mathbf{v}^{\prime}-\mathbf{T}^{\prime} \mathbf{u}^{\prime}=\delta^{\prime}
$$

The relations between the matrices $\mathbf{T}^{\prime}$ and $\boldsymbol{\delta}^{\prime}$, on the one hand, and $\mathbf{T}$ and $\delta$, on the other hand, are easily derived by using Eqs. 7.12 and 7.13 in Eq. 5.2 above. We obtain

$$
\begin{gathered}
\mathbf{T}^{\prime}=\mathbf{R T R}^{-1} \\
\delta^{\prime}=\mathbf{R} \delta=\frac{1}{2}\left[\begin{array}{l}
\delta_{1}+\delta_{2} \\
\delta_{1}-\delta_{2}
\end{array}\right]=\left[\begin{array}{l}
\delta_{1}^{\prime} \\
\delta_{2}^{\prime}
\end{array}\right]
\end{gathered}
$$

Let us now rewrite the noise measure, Eq. 6.25, in terms of the matrices $\mathbf{T}^{\prime}$ and $\boldsymbol{\delta}^{\prime}$. For this purpose we note that, from the definition of Eq. 7.11 for $\mathbf{R}$, we have

$$
\mathbf{R}=\mathbf{R}^{\dagger}=\frac{1}{2} \mathbf{R}^{-1}
$$

Using Eqs. 7.16 and 7.17, we can write the noise matrix $\overline{8 \delta^{\dagger}}$ as

$$
\overline{\delta \delta^{\dagger}}=\mathbf{R}^{-1} \overline{\delta^{\prime} \delta^{\prime \dagger}} \mathbf{R}^{-1}=4 \mathbf{R} \overline{\delta^{\prime} \delta^{\prime \dagger}} \mathbf{R}^{\dagger}
$$

Furthermore, we have from Eqs. 7.15 and 7.17

$$
\begin{aligned}
\mathbf{P}-\mathbf{T P T}^{\dagger} & =\mathbf{P}-\mathbf{R}^{-1} \mathbf{T}^{\prime} \mathbf{R} \mathbf{P} \mathbf{R}^{\dagger} \mathbf{T}^{\prime \dagger}\left(\mathbf{R}^{-1}\right)^{\dagger} \\
& =2 \mathbf{R}\left[2 \mathbf{R} \mathbf{P} \mathbf{R}^{\dagger}-\mathbf{T}^{\prime}\left(2 \mathbf{R} \mathbf{P} \mathbf{R}^{\dagger}\right) \mathbf{T}^{\prime \dagger}\right] \mathbf{R}^{\dagger} \\
& =2 \mathbf{R}\left(\mathbf{P}^{\prime}-\mathbf{T}^{\prime} \mathbf{P}^{\prime} \mathbf{T}^{\prime \dagger}\right) \mathbf{R}^{\dagger}
\end{aligned}
$$


where

$$
\mathbf{P}^{\prime}=2 \mathbf{R} \mathbf{P} \mathbf{R}^{\dagger}=\mathbf{R} \mathbf{P} \mathbf{R}^{-1}=\left[\begin{array}{rr}
1 & 0 \\
0 & -1
\end{array}\right]
$$

Combining Eqs. 7.18 and 7.19, we obtain for the noise measure, Eq. 6.25,

$$
M_{e}=\frac{\mathbf{w}^{\dagger} \delta^{\prime} \delta^{\prime \dagger} \mathbf{w}}{\mathbf{w}^{\dagger}\left(\mathbf{P}^{\prime}-\mathbf{T}^{\prime} \mathbf{P}^{\prime} \mathbf{T}^{\prime \dagger}\right) \mathbf{w}} \frac{1}{k T_{0} \Delta f}
$$

with

$$
\mathbf{w}=\left[\begin{array}{l}
w_{1} \\
w_{2}
\end{array}\right]=\mathbf{R}^{\dagger} \mathbf{y}=\frac{1}{2}\left[\begin{array}{l}
y_{1}+y_{2} \\
y_{1}-y_{2}
\end{array}\right]
$$

The noise measure expressed in terms of the voltage and current variables is optimized when $\mathbf{y}$ is an eigenvector of the noise matrix N. Correspondingly, the noise measure is also optimized in terms of the wave formulation if $\mathbf{w}$ is an eigenvector of the noise matrix

$$
\mathbf{N}^{\prime}=\left(\mathbf{P}^{\prime}-\mathbf{T}^{\prime} \mathbf{P}^{\prime} \mathbf{T}^{\prime \dagger}\right)^{-1} \overline{\delta^{\prime} \delta^{\prime \dagger}}
$$

The requirement that $\operatorname{Re}\left(y_{2}{ }^{(1)} / y_{1}{ }^{(1)}\right)>0$ imposed on the eigenvector $\mathbf{y}^{(1)}$ imposes a corresponding limitation on $\mathbf{w}^{(1)}$. From Eq. 7.21,

$$
\frac{w_{2}}{w_{1}}=-\left(\frac{\frac{y_{2}}{y_{1}}-1}{\frac{y_{2}}{y_{1}}+1}\right)=-\left(\frac{Z_{S}^{*}-1}{Z_{S}^{*}+1}\right)
$$

Thus, $w_{2} / w_{1}$ is the negative-conjugate reflection coefficient corresponding to the source impedance $Z_{S}$. Therefore, we must have

$$
\left|\frac{w_{2}}{w_{1}}\right|<1
$$

From Eqs. 7.15, 7.16, 7.19, and 7.22, it is easily found that, for the network of Fig. 7.1a,

$$
\mathbf{N}^{\prime}=\left[\begin{array}{cc}
\frac{|u|^{2} \sqrt{\left.\delta_{1}{ }^{\prime}\right|^{2}}}{|u|^{2}-1} & \frac{|u|^{2} \overline{\delta_{1}{ }^{\prime} \delta_{2}{ }^{\prime *}}}{|u|^{2}-1} \\
-\overline{\delta_{1}{ }^{\prime *} \delta_{2}{ }^{\prime}} & -\overline{\left.\delta_{2}{ }^{\prime 2}\right|^{2}}
\end{array}\right]
$$


The positive eigenvalue of $\mathbf{N}^{\prime}$ is

$$
\begin{aligned}
\lambda_{1}= & \frac{1}{2}\left[\frac{|u|^{2}}{|u|^{2}-1} \overline{\left.\delta_{1}{ }^{\prime}\right|^{2}}-\overline{\left.\delta_{2}{ }^{\prime}\right|^{2}}\right. \\
& \left.+\sqrt{\left(\frac{|u|^{2}}{|u|^{2}-1} \overline{\left|\delta_{1}{ }^{\prime}\right|^{2}}-\sqrt{\left|\delta_{2}{ }^{\prime}\right|^{2}}\right)^{2}+4 \frac{|u|^{2}}{|u|^{2}-1}\left(\left|\overline{\delta_{1}{ }^{\prime}{ }^{2}}\right| \overline{\left.\delta_{2}{ }^{\prime}\right|^{2}}-\left|\overline{\delta_{1}{ }^{\prime} \delta_{2}{ }^{\prime *}}\right|^{2}\right)}\right]
\end{aligned}
$$

From the definition of the eigenvector, the matrix equation results:

$$
\mathbf{N}^{\prime} \mathbf{w}^{(1)}=\lambda_{1} \mathbf{w}^{(1)}
$$

of which the second component is

and thus

$$
-\overline{\delta_{1}{ }^{*} \delta_{2}{ }^{\prime} w_{1}^{(1)}}-\overline{\delta_{2}{ }^{\prime 2}} w_{2}^{(1)}=\lambda_{1} w_{2}^{(1)}
$$

$\frac{w_{2}^{(1)}}{w_{1}^{(1)}}$

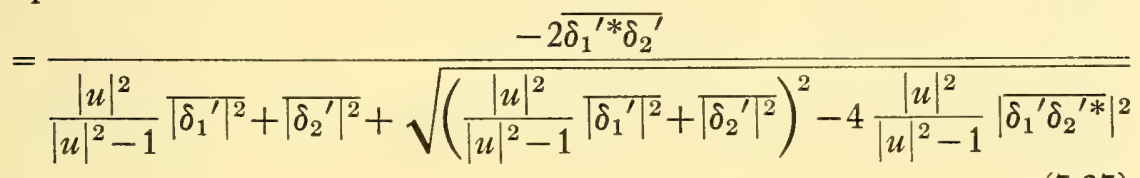

But $|u|>1$, and the eigenvalue $\lambda_{1}$ is real. Therefore, from Eq. 7.26

$$
4\left|\overline{\delta_{1}{ }^{\prime} \delta_{2}{ }^{\prime *}}\right|^{2}<4 \frac{|u|^{2}}{|u|^{2}-1}\left|\overline{\delta_{1}{ }^{\prime} \delta_{2}{ }^{\prime *}}\right|^{2}<\left(\frac{|u|^{2}}{|u|^{2}-1} \sqrt{\left.\delta_{1}{ }^{\prime}\right|^{2}}+\overline{\left.\delta_{2}{ }^{\prime 2}\right|^{2}}\right)^{2}
$$

which, in Eq. 7.27 yields

$$
\left|\frac{w_{2}^{(1)}}{w_{1}{ }^{(1)}}\right|<1
$$

The eigenvector $\mathbf{w}^{(1)}$ for which the noise measure is optimized thus corresponds to a reflection coefficient less than unity, that is, to a passive source impedance. The condition in Eq. 7.28 is equivalent to the condition, Eq. 7.10, in the general-circuit-matrix notation. We have thus proved that the noise measure of a unilateral amplifier can be optimized with a lossless mismatching network between the source (having an impedance with a positive real part) and the amplifier. The output impedance of this amplifier always has a positive real part. It follows that any number of optimized amplifiers, with $\operatorname{det}\left(\mathbf{P}-\mathbf{T} \mathbf{P} \mathbf{T}^{\dagger}\right)<0$, can be cascaded with appropriate lossless mismatching networks between successive stages so as to achieve an arbitrarily high gain. The excess-noise 
figure of the cascade is then equal to the minimum noise measure of each stage, which, with the aid of Eq. 7.8 for $\mathbf{N}$, is

$$
\begin{aligned}
& M_{e, \mathrm{opt}}=\frac{\lambda_{1}}{k T_{0} \Delta f}=\frac{1}{8 k T_{0} \Delta f}\left[\frac{\overline{\left|E_{n 1}\right|^{2}}+\mid \overline{\left.I_{n 1}\right|^{2}}}{|u|^{2}-1}+\frac{2|u|^{2}-1}{|u|^{2}-1} 2 \operatorname{Re} \overline{E_{n 1} I_{n 1}{ }^{*}}\right. \\
& +\sqrt{\left.\begin{array}{c}
\left(\frac{\left|E_{n 1}\right|^{2}+\sqrt{\left.I_{n 1}\right|^{2}}}{|u|^{2}-1}+\frac{2|u|^{2}-1}{|u|^{2}-1} 2 \operatorname{Re}\left(\overline{E_{n 1} I_{n 1}{ }^{*}}\right)\right)^{2} \\
+16 \frac{|u|^{2}}{|u|^{2}-1}\left(\left.\overline{\left|E_{n 1}\right|^{2}}\left|\overline{\left.I_{n 1}\right|^{2}}-\right| \overline{E_{n 1} I_{n 1}{ }^{*}}\right|^{2}\right)
\end{array}\right]}
\end{aligned}
$$

The proof of the inequality in Eq. 7.28 can easily be extended to cover the case of nonunilateralized amplifiers of the class of Fig. 7.1a, provided they have passive conjugate-image impedances. We start with the network in the form that has the scattering matrix

with

$$
\mathbf{S}=\left[\begin{array}{cc}
0 & S_{12} \\
S_{21} & 0
\end{array}\right]
$$

$$
\left|S_{21}\right|>1
$$

and

$$
\left|S_{12}\right|<1
$$

The only differences occur as minor modifications in Eqs. $7.25 \mathrm{ff}$., where $\left|S_{21}\right|^{2}$ replaces $|u|^{2}$ and $\left(1-\left|S_{12}\right|^{2}\right)$ appears in other terms. Thus, unilateralization is not a necessary step to achieve optimum noise measure with input mismatch. Amplifiers that have passive conjugate-image impedances can be optimized for noise measure by an input mismatch alone. However, the output impedance under optimized conditions is guaranteed to have a positive real part only if the amplifier is also stable under arbitrary passive input and output loading. Most vacuum-tube and transistor amplifiers meet these conditions over a significant frequency range.

\subsection{The Optimum Noise-Measure Expression for the Conventional Low-Frequency Vacuum Tube}

We shall now derive from Eq. 7.29 the expression for the minimum noise measure of a conventional low-frequency vacuum tube. The noise in the tube is characterized by a grid noise resistance $R_{n}$, the input impedance is $R_{1}$, and the plate resistance is $r_{p}$. The noise-voltage column 
matrix is

$$
\mathbf{E}=\left[\begin{array}{l}
E_{1} \\
E_{2}
\end{array}\right]
$$

with

$$
\overline{\left.E_{2}\right|^{2}}=4 k T_{0} \Delta f\left(R_{n}+R_{1}\right) \mu^{2}
$$

and

$$
\begin{aligned}
\overline{E_{1}{ }^{2}} & =4 k T_{0} \Delta f R_{1} \\
\overline{E_{1} E_{2}{ }^{*}} & =-4 k T_{0} \Delta f \mu R_{1}
\end{aligned}
$$

The impedance matrix is

$$
\left[\begin{array}{c:c}
R_{1} & 0 \\
\hdashline-\mu R_{1} & r_{p}
\end{array}\right]
$$

In order to make the impedance matrix of the triode represent the normalized form (Fig. 7.1a), suitable ideal transformers have to be connected to the input and output. The new impedance matrix then becomes

$$
\mathbf{Z}^{\prime}=\left[\begin{array}{cc}
1 & 0 \\
-\mu \sqrt{\frac{R_{1}}{r_{p}}} & 1
\end{array}\right]
$$

and the new open-circuit noise voltages of the over-all network are

$$
\mathbf{E}^{\prime}=\left[\begin{array}{c}
\frac{E_{1}}{\sqrt{R_{1}}} \\
\frac{E_{2}}{\sqrt{r_{p}}}
\end{array}\right]
$$

Finally, in general-circuit-parameter form, the parameters of the triode become

$$
\mathbf{T}=-\frac{1}{2 u}\left[\begin{array}{ll}
1 & 1 \\
1 & 1
\end{array}\right]
$$

where

Also

$$
u=\frac{1}{2} \mu \sqrt{\frac{R_{1}}{r_{p}}}
$$

$$
\delta=\frac{1}{2 u}\left[\begin{array}{c}
\frac{E_{2}}{\sqrt{r_{p}}}+\frac{2 u E_{1}}{\sqrt{R_{1}}} \\
\frac{E_{2}}{\sqrt{r_{p}}}
\end{array}\right]=\left[\begin{array}{c}
E_{n 1} \\
I_{n 1}
\end{array}\right]
$$


With these specific values we obtain for the optimum noise-measure expression of the triode

$$
M_{\mathrm{opt}}=2\left[\frac{R_{n}+\frac{r_{p}}{\mu^{2}}+\sqrt{R_{n}{ }^{2}+R_{1} R_{n}+\frac{r_{p}{ }^{2}}{\mu^{4}}-2 \frac{r_{p}}{\mu^{2}} R_{n}}}{R_{1}-\frac{4 r_{p}}{\mu^{2}}}\right]
$$

For large values of $\mu$, the minimum noise measure is effectively equal to the minimum excess-noise figure, and all terms in Eq. 7.37 divided by $\mu$ can be disregarded. We obtain in the limit $\mu \rightarrow \infty$

$$
F_{\min }=1+\frac{2}{R_{1}}\left(R_{n}+\sqrt{R_{n}{ }^{2}+R_{1} R_{n}}\right)
$$

This result is well known. ${ }^{2}$

\subsection{Optimization of Negative-Resistance Amplifiers, Definite Case}

There remains the problem of achieving the optimum noise measure of negative-resistance amplifiers, that is, the class illustrated in Fig. 7.1c. This problem we now wish to solve, employing a positive source impedance and guaranteeing that a positive output impedance results.

While it is actually possible to accomplish our purpose by performing a consecutive series of lossless reciprocal imbeddings, starting from the specific amplifier form given in Fig. 7.1c, the particular method we found for doing it was rather involved. It was also of little interest beyond its application to the present proof.

Fortunately, there exists another method of optimizing the noise performance of any nonpassive network, including negative-resistance networks. This method is not only simple analytically but has a practical bearing upon the noise optimization of the new maser amplifier. We shall present this solution and its relation to the maser.

We have shown in Chap. 4 that every two-terminal-pair network can be reduced by lossless nonreciprocal imbedding to the canonical form of Fig. 7.2, comprising two isolated (positive or negative) resistances in series with uncorrelated noise voltage generators. Moreover, the opencircuit noise voltages $E_{n 1}$ and $E_{n 2}$ and the two eigenvalues $\lambda_{1}$ and $\lambda_{2}$ of the characteristic-noise matrix $\mathbf{N}$ are directly related:

$$
\frac{\overline{E_{n 1}^{2}}}{4 R_{1}}=-\lambda_{1} ; \quad \frac{\overline{E_{n 2}^{2}}}{4 R_{2}}=-\lambda_{2}
$$

${ }^{2}$ A. van der Ziel, Noise, Prentice-Hall, New York (1954). 
In the special case of a negative-resistance amplifier, the eigenvalues $\lambda_{1}$ and $\lambda_{2}$ are both positive. Accordingly, resistances $R_{1}$ and $R_{2}$ of the canonical form are both negative. We suppose now that the eigenvalue $\lambda_{1}$ has the smaller magnitude. According to the theory of Chap. 6, this

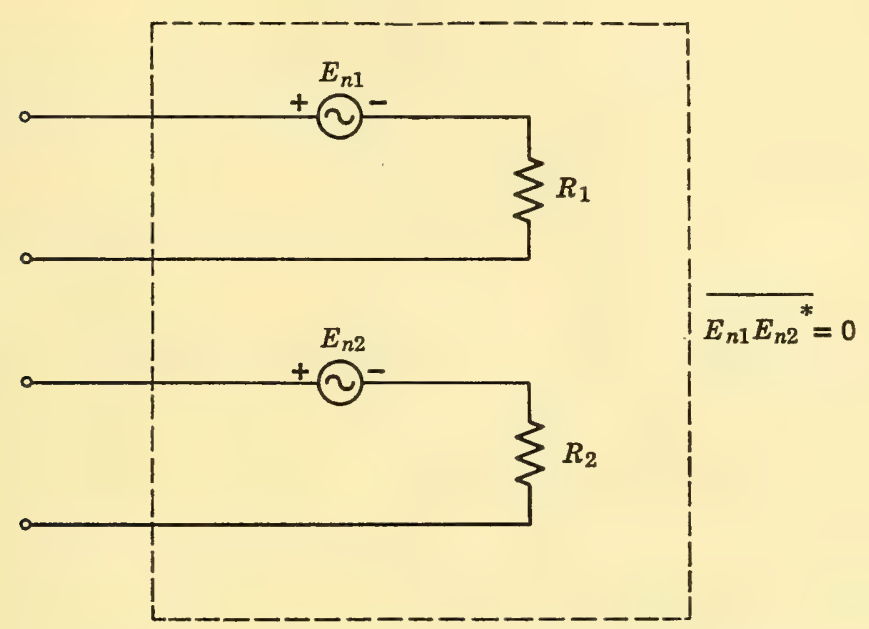

Fig. 7.2. Canonical form of two-terminal-pair amplifier.

eigenvalue determines the lowest achievable value of the noise measure $M_{e}$. We shall now prove that this lowest value, $\lambda_{1} /\left(k T_{0} \Delta f\right)$, can indeed be achieved using only that terminal pair of Fig. 7.2 which contains the negative resistance $R_{1}$ and noise generator $E_{n 1}$.

As shown in Fig. 7.3, the terminal pair $\left(R_{1}, E_{n 1}\right)$ of the canonical form is connected to terminal pair 2 of an ideal lossless circulator with the scattering matrix

$$
\mathbf{S}=\left[\begin{array}{llll}
0 & 0 & 0 & 1 \\
1 & 0 & 0 & 0 \\
0 & 1 & 0 & 0 \\
0 & 0 & 1 & 0
\end{array}\right]
$$

(Transmission lines with 1-ohm characteristic impedance are connected to all four terminal pairs of the circulator.) Terminal pair (4) of the circulator is matched to a $1-\mathrm{ohm}$ load at a temperature $T_{0}$, terminal pair (1) is used as the input, and terminal pair (3) is used as the output (Fig. 7.3).

The equations for the resulting two-terminal-pair network can easily be derived using the scattering-matrix representation. We find that the 


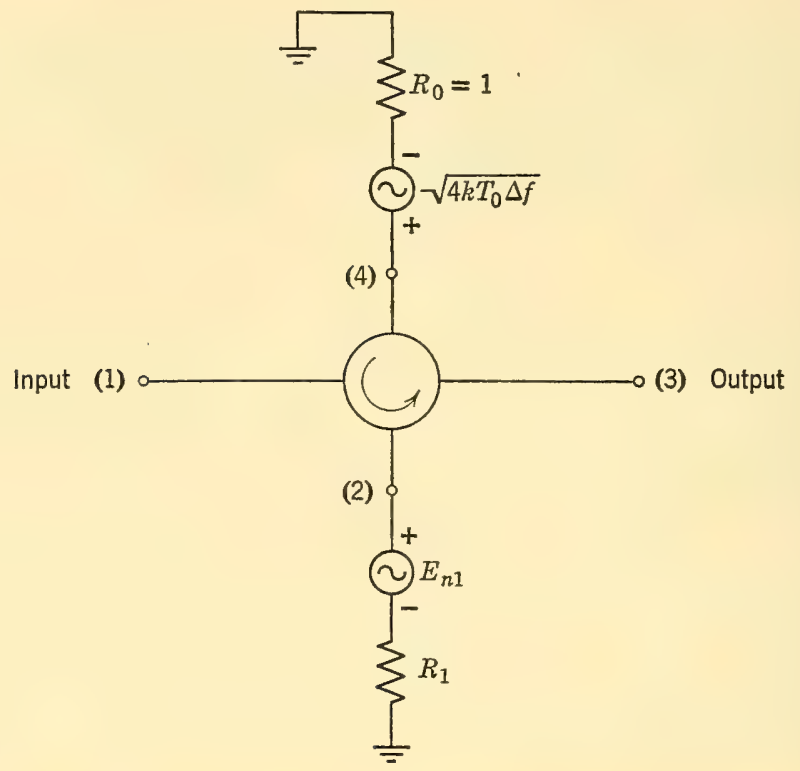

Fig. 7.3. Realization of optimum amplifier noise performance from canonical form of the amplifier.

amplifier is unilateral and is described by the equations:

$$
\begin{aligned}
& a_{1}=\frac{R_{1}+1}{R_{1}-1} b_{3}+\delta_{1}{ }^{\prime} \\
& b_{1}=\delta_{2}{ }^{\prime}
\end{aligned}
$$

where

$$
\begin{aligned}
\overline{\left.\delta_{2}{ }^{\prime}\right|^{2}} & =k T_{0} \Delta f \\
\overline{\left.\delta_{1}{ }^{\prime}\right|^{2}} & =\frac{\mid \overline{\left.E_{n 1}\right|^{2}}}{\left(1-R_{1}\right)^{2}} \\
\overline{\delta_{1}{ }^{\prime} \delta_{2}{ }^{\prime *}} & =0
\end{aligned}
$$

The available gain $G$ of the amplifier with a $1-\mathrm{ohm}$ source is

$$
G=\left(\frac{R_{1}-1}{R_{1}+1}\right)^{2}
$$

The excess-noise figure of the resulting amplifier is

$$
F-1=\frac{\sqrt{\delta_{1}{ }^{2}}{ }^{2}}{k T_{0} \Delta f}
$$


Thus, according to the foregoing results and Eq. 7.38,

$$
M=M_{e}=\frac{F-1}{1-\frac{1}{G}}=-\frac{\overline{\left.E_{n 1}\right|^{2}}}{4 R_{1} k T_{0} \Delta f}=\frac{\lambda_{1}}{k T_{0} \Delta f}
$$

We have therefore proved that the circulator arrangement indeed achieves the lowest possible noise measure. Since it also leads to a unilateral amplifier with positive real input and output impedances, an arbitrary gain can be achieved through cascading of such identical amplifiers. We observe, however, that a lossy network (ideal lossless circulator plus $R_{0}$ ) has been employed with the original amplifier to optimize its noise performance.

The optimization carried out in connection with Fig. 7.3 has a useful corollary concerning circuit connections of maser amplifiers for optimum noise performance. One of the forms of the maser has for an equivalent circuit a one-terminal-pair negative resistance $R_{1}$ in series with a noise voltage generator $E_{n 1}$. To make a two-terminal-pair network, we may consider as an artifice not only the noisy negative resistance $R_{1}$ of the maser but also another positive resistance $R_{2}$ at a temperature $T_{2}$. The two resistances can be treated as the canonical form of a two-terminal-pair network. Lossless imbedding of these two resistances therefore leads to a two-terminal-pair amplifier with the eigenvalues $\lambda_{1}=-\left|E_{n 1}\right|^{2} /\left(4 R_{1}\right)>0$ and $\lambda_{2}=-k T_{2} \Delta f<0$. The best noise measure that can be expected from the resulting amplifier is $M_{\mathrm{opt}}=\lambda_{1} /\left(k T_{0} \Delta f\right)$. The circulator arrangement has been shown to achieve this noise measure. Thus, it provides one of the optimum network connections of the maser with regard to noise performance. It should be re-emphasized that the assumed presence of a positive resistance $R_{2}$ in the circuit is an artifice that enables the use of the theory of two-terminal-pair networks for the noise study of the one-terminal-pair maser. The assumed temperature of the resistance is immaterial because it determines only the negative eigenvalue of the characteristic-noise matrix, which has no relation to the optimum noise measure achieved with gain.

The results of this chapter lead to the following theorem:

1. Any unilateral amplifier with $U>1$ may be optimized with input mismatch alone.

2. A nomunilateral amplifier with $U>1$, which is also stable for all passive source and load impedances, may be optimized with input mismatch alone.

3. Any amplifier with $U>1$ may be optimized by first making it unilateral, using lossless reciprocal networks, and subsequently employing input mismatch. 
4. Amplifiers of the class $U<0$ can be optimized by first transforming them into the class $U>1$ by lossless nonreciprocal imbedding. The optimization methods 1 to 3 can then be applied to this class.

5. Negative-resistance amplifiers $(0<U<1)$ can be optimized by first transforming them into the canonical form. The terminal pair of the canonical form that possesses the exchangeable power of smaller magnitude is connected into a lossless circulator with a positive (1-ohm) balancing resistor, as shown in Fig. 7.3. The resulting unilateral two-terminal-pair network, driven from a 1-ohm source, achieves the optimum noise measure. 


\section{8}

\section{Conclusions}

The developments that we have undertaken have been rather lengthy. Therefore, it is worth while to reassess and summarize our principal results as well as our omissions.

As pointed out in the introduction, the original motivation for the present work was the desire to describe in a systematic manner the singlefrequency noise performance of two-terminal-pair linear amplifiers. It was necessary at the outset to elect a criterion of noise performance, which we chose to be the signal-to-noise ratio achievable at high gain. This criterion is not clear for systems without gain, nor for multiterminal-pair networks. For multiterminal-pair networks, the noise parameter $p_{T}$ expressed in terms of the general circuit constants has been set down as an extension of the two-terminal-pair noise-measure definition but has not been given any physical interpretation in this work. One reason for this omission is the fact that a general-circuit-constant (or wave-matrix) description of multiterminal-pair systems has been of little use in the past. There have not been any systems incorporating gain whose noise performance on a multiterminal-pair basis was of interest. It is true that in the past some special problems involving frequency conversion have called for proper interpretations, and that two-terminal-pair networks processing sidebands may be analyzed theoretically as multiterminal-pair networks. But a sophisticated theoretical approach to noise problems of this nature was never necessary. Problems of this type were easily disposed of by inspection.

Recently, parametric amplifiers (nonlinear-, or time-varying-, reactance amplifiers) have received a great deal of attention because of their low- 
noise characteristics. In parametric amplifiers correlation between signal sidebands often occurs and must be taken into account in the mathematical analysis. For such an analysis a systematic theory of noise in multiterminal-pair networks involving correlation between signal and/or noise sidebands is required. It is also probable that, because of these very same signal correlations between sidebands, the appropriate theory for the parametric case may not be merely the theory of the generalized noise parameter $p_{T}$ introduced in this work. We have not had the opportunity to pursue this interesting question in much detail. But we have studied the question sufficiently to be convinced that the general matrix methods of dealing with power and power ratios employed in the present study will help greatly in the analysis and understanding of these somewhat more difficult problems.

The remarks of the previous paragraphs do not, of course, imply that multiterminal-pair networks have been neglected completely. Indeed, in the impedance-matrix formulation we have given extensive attention to the exchangeable-power interpretations of the network invariants in the multiterminal-pair case. As a practical application of these ideas, we may refer again to the work of Granlund ${ }^{1}$ regarding the problem of combining a multiplicity of antenna outputs into a single receiver, when the inputs to the antennas are statistically related. Furthermore, from the impedance formulation we have been able to develop a canonical form for the multiterminal-pair network. The merit of this form is that it leads to a simplification in thinking about single-frequency noise and gain characteristics of linear networks.

The problem of considering noise performance over a broad band, rather than at a single frequency, appears to be covered by the spot-noise discussions that we have conducted. Certainly, in a two-terminal-pair amplifier one could adopt the position of optimizing the noise measure at each frequency in the band. Although such a procedure might involve complicated feedback variations with frequency and/or intricate matching systems, these are principally network-synthesis problems that presumably could be solved on the basis of suitable approximations, if it appeared desirable to do so. There is no doubt that such a solution would give the "optimum noise performance" of the amplifier. By this we mean that the optimum is to be interpreted as the "best signal-to-noise ratio at high gain, at each frequency within the band." It is by no means obvious that, with the over-all system in mind, such a solution is always the best. There are many other considerations besides noise performance which enter into the design of wideband amplifiers, such as the behavior

${ }^{1}$ J. Granlund, Topics in the Design of Antennas for Scatter, M.I.T. Lincoln Laboratory Technical Report 135, Massachusetts Institute of Technology, Cambridge, Mass. (1956). 
of the phase characteristic, transient response, and the uniformity of the gain-to say nothing of over-all circuit complexity. Therefore, it seems clear that no general theory of noise performance of such wideband systems should be undertaken without attention to other system requirements.

The last point brings us to the question of the usefulness of an optimum noise-performance criterion of the type we have presented in this study. It is probable that such a criterion will serve primarily as an indication of the extent to which a given design, which has met a variety of other practical conditions, fails to achieve its best noise performance. In other words, one may very well not attempt to realize the optimum noise measure directly but use it instead as a guide to detect the onset of diminishing returns in further efforts to improve noise performance. 



\section{Index}

Amplifier, negative-resistance, 61, 68, 72 nonunilateral, 66, 71

parametric, 73

two-terminal-pair, classification of, 59

$U<0,72$

unilateral, 71

unilateral $U>1,71$

wideband, 74

Amplifier cascade, 2

Amplifier noise performance, criterion for, 5

Amplifiers, interconnection of, 55

Available power, 14

Becking, A. G. Th., 46, 47

Belevitch, V., 33

Canonical form, 31 derivation of, 28

Cascading, problem of, 3

Characteristic-noise matrix, eigenvalues of, 23 general formulation of, 38

impedance formulation of, 22

mixed voltage-current formulation of, 41

trace of, 23

Circulator, 69, 72

Classification of networks and eigenvalues, 24 in $\mathrm{T}$-matrix representation, 40
Classification of two-terminal-pair amplifiers, 59

Cross-power spectral densities, 10

Dahlke, W., 46, 47

Eigenvalues, 22 classification of, 24

least positive, 53

Excess-noise figure, 48 lower limit imposed on, 53

Exchangeable power, 15, 44 matrix form for, 19 matrix formulation of, 44 n-terminal-pair networks, 15 stationary values of, 22

Exchangeable-power gain, 43, 46 algebraic signs of, 49

Extended noise figure, 43, 46

Feedback, 3

lossless, 54

Franz, K., 2

Friis, H. T., 2

Gain, available, 43 exchangeable-power, 43,46 
Gain, extended definitions of, 43 unilateral $U, 59$

General-circuit-parameter representation, 36, 61

Granlund, J., 17, 74

Grid-noise resistance, 66

Groendijk, H., 46

Gyrator, lossless, 59

Hermitian conjugate, 11

Hermitian matrix, 11

Imbedding, 12

lossless, 20

passive dissipative, $\mathbf{5 7}$

reciprocal, 59

Impedance representation, 6, 9, 38

Indefinite matrix, 16, 52

Interconnection of amplifiers, lossless, 6, 54 passive dissipative, $\mathbf{5 5}$

Invariants, 25, 31

Knol, K. S., 46

Lossless circulator, 69

Lossless feedback, 54

Lossless gyrator, 59

Lossless imbedding, 20

Lossless interconnection, 54

Lossless transformation, 9, 12, 19, 61

Losslessness, condition of, 13

Maser, 7, 68

Mason, S. J., 3, 59, 61

Matrix, characteristic-noise, 22, 38, 41

general-circuit, 61

general representation, 34

Hermitian, 11

impedance, 9

indefinite, 16,52

negative definite, 16,52

noise column, 47,61

permutation, 45

positive definite, $11,16,52$

scattering, 69

semidefinite, 11,16

T, 34, 39, 40

M atrix formulation, of exchangeable power, 44

of exchangeable-power gain, 45

of extended noise figure, 46
Matrix formulation, of stationary-value problem, 19

Mismatch, input, 6, 54, 61, 71

$n$-to- $n$-terminal-pair network transformations, 6, 25

$n$-to-one-terminal-pair network transformation, 6,18

Negative definite matrix, 16, 52

Network transformations, $n$-to- $n$-terminalpair, 6, 25

$n$-to-one-terminal-pair, 6, 18

Networks, classification of, 14, 24, 40

in thermal equilibrium, 25

pure-noise, 47

with coherent sources, 25

Noise column matrix, 47,61

Noise figure, excess, 48

extended, 43, 46

matrix formulation of, 46

Noise measure, $4,42,48$

allowed ranges of, 49

for conventional low-frequency vacuum tube, 66

for triode, 66

optimum $\left(M_{e, \text { opt }}\right), 54,55$

Noise parameter $p_{T}, 39$

in mixed voltage-current representation, 41

Noise performance, criterion for amplifier, 5 optimization of, 61,68

single-frequency, 1, 73

spot, 1

Nonreciprocal transformations, 59

Nonunilateral amplifier, 66, 71

Optimization, of amplifier, indefinite case, 61 of maser amplifiers, 71

of negative-resistance amplifiers, definite case, 68

Optimum noise measure $\left(M_{e, \text { opt }}\right), 54,55$

for low-frequency vacuum tube, 66

Parametric amplifier, 73

Passive dissipative imbedding, 56

Passive dissipative interconnection of amplifiers, 56

Passive network at equilibrium, 26

Permutation matrix, 45

Positive definite matrix, 11, 16, 52

Power, available, 14

exchangeable, 15, 44 
Power spectral densities, 11

Reciprocal imbedding, 59

Reduction of number of terminal pairs, 32, 56

Robinson, F. N. H., 3

Rothe, H., 46, 47

Scattering-matrix representation, 69

Self-power spectral densities, 10

Semidefinite matrix, 11, 16

Signal-to-noise ratio, 1, 73

Single-frequency noise performance, 1, 73

Spectral densities, cross-power, 10 power, 11 self-power, 10

Spot-noise figure, 2

Stationary-value problem, eigenvalue formulation of, 21 matrix formulation of, 19

Stationary values of exchangeable power, 22
T-matrix representation, $34,39,40$

Terminal-voltage and current vector, 38

Thévenin representation, 14

Trace of characteristic-noise matrix, 23

Transformation, lossless, 9, 12, 19, 61 $n$-to- $n$-terminal-pair network, 6,25

$n$-to-one-terminal-pair network, 6, 18

Transformation from one matrix representation to another, 35

Transformation network, 12

Transistor amplifiers, 59

Twiss, R. Q., 26

Unilateral amplifier, 71

Unilateral amplifier with $U>1,71$

Unilateral gain $U, 59$

Vacuum tube, 59, 66

van der Ziel, A., 2, 68

Vector, noise column, 47, 66

Wideband amplifiers, 74 






WUULo rivle

OCEANOGRAPHIC INSTITUTION

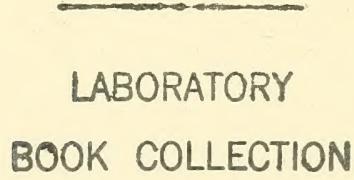



